European

Surgical

Research
Eur Surg Res 2016;57:34-80

DOI: 10.1159/000445224

Published online: April 20, 2016

\title{
133rd Congress of the German Society of Surgery (DGCH)
}

April 26-29, 2016, Berlin, Germany

Abstracts

Guest Editor

Gabriele Schackert, Dresden

The abstracts are available online, free of charge, under http://www.karger.com/Article/FullText/445224 


\section{Abstracts}

\section{Role of ENTPD2 in Liver Regeneration and Liver Fibrosis}

\author{
L. Feldbruegge ${ }^{1}$, S. Mitsuhashi ${ }^{1}$, E. Csizmadia ${ }^{1}$, \\ M. Schmelzle ${ }^{2}$, S. Robson ${ }^{1}$ \\ ${ }^{1}$ Beth Israel Deaconess Medical Center, Boston, USA \\ ${ }^{2}$ Charité, Berlin, Germany
}

Background: Ecto-nucleoside triphosphate diphosphohydrolases (ENTPD) comprise a family of cell surface located transmembrane proteins that regulate purinergic signaling by catalyzing extracellular nucleotides, such as ATP and ADP. The prototype ENTPD1/CD39 is expressed on endothelium and sinusoidal immune cells, and is a regulator of liver regeneration. The closely related enzyme ENTPD2 is expressed on portal fibroblasts and perivascular cells. In vitro co-incubation studies show that this enzyme on fibroblasts regulates proliferation of cholangiocytes. Its role in liver function in vivo however, has not yet been determined. Herein, we examine pathophysiological functions of ENTPD2 in liver regeneration and liver fibrosis using a newly generated ENTPD2 deficient mouse line.

Materials and Methods: Global ENTPD2 knockout mice and matched wild type mice were subjected to $2 / 3$ hepatectomy as a model for liver regeneration, and to 6 weeks of carbon tetrachloride ( $\mathrm{CCl} 4)$ treatment to induce liver fibrosis. Outcomes were compared by studying serum liver function tests, liver histology and immunohistochemistry. Collagen content of liver tissue was determined biochemically by measuring relative hydroxyproline content.

Results: ENTPD2 null mice develop normally on the C57BL6 background and do not show abnormalities in liver morphology at baseline. After partial hepatectomy, hepatic ENTPD2 mRNA expression in wild type mice decreased in the first 24 hours and then significantly increased to 3 times basal level at day 5. Serum ALT and other parameters of liver injury as well as the rate of hepatocellular proliferation were comparable in both groups, and there was no relevant difference in liver mass regeneration. However, when fibrosis was induced by $\mathrm{CCl} 4$ treatment, mice deficient for ENTPD2 showed significantly more collagen deposition as measured by hydroxyproline content. Histological analysis confirmed more severe fibrosis in the knockout mice while there was no significant difference in liver function tests.

Conclusion: ENTPD2 null mice show no major hepatic developmental abnormalities and appear to maintain normal liver regeneration responses when subjected to the acute injury of partial hepatectomy in otherwise healthy livers. In the setting of chronic hepatocellular injury by $\mathrm{CCl} 4$ ingestion however, ENTPD2 deficient mice show significantly more fibrosis. As this ecto-enzyme is expressed by myofibroblastic cells surrounding bile ductules within portal triads, and deletion promotes fibrogenesis, we propose that ATP scavenging by ENTPD2 might represent a novel therapeutic target in fibrotic liver disease.

\section{2 \\ Platelets Promote CD133+BMSC Adhesion to Murine Micro and Hepatic Sinusoidal Endothelium Independent of ADP Stimulation Under Flow Condition \\ J. Kirchner ${ }^{1}$, C. Duhme ${ }^{1}$, N. Lehwald ${ }^{1}$, K. Wieferich ${ }^{1}$, M. Schmelzle ${ }^{2}$, N.H. Stoecklein ${ }^{1}$, A. Krieg ${ }^{1}$, W.T. Knoefel', J. Schulte am Esch ${ }^{1}$ \\ ${ }^{1}$ Universitätsklinikum der Heinrich Heine Universität Düsseldorf, Düsseldorf, ${ }^{2}$ Chirurgische Kliniken der Charité am Campus Virchow-Klinikum, Berlin, Germany}

Background: We previously demonstrated the therapeutic potential of hematopoetic CD133+ bone marrow stem cells (CD133+BMSC) to promote clinical liver regeneration and the potential of platelets for CD133+BMSC homing along human micro endothelium under shear stress. In this study, we have established a murine model to gain the option of investigation of null and overexpressing cells for homing relevant factors. Furthermore, we evaluated a putative adhesion promoting effect of platelets for CD133+BMSC-homing along hepatic sinusoidal vasculature.

Materials and Methods: Commercially available mouse primary dermal micro vasculature endothelial cells (dMECC57BL/6) and mouse hepatic sinusoidal endothelial cells (mSEC) were cultured in capillaries in a live cell imaging system (Bioflux). Primary CD133+BMSC were purified from bone marrow flushed from murine tibiae and femori utilizing FACsorting. dMEC or mSEC were co-cultured under shear stress with CD133+BMSC and platelet rich plasma (PRP) or platelet poor plasma (PPP) as control prepared from murine BMSC donors. To test the effect of pre-stimulation of platelets for adhesion of CD133+BMSC, murine PRP was pre-stimulated with ADP monitored by aggregometry utilizing the Bohrlight-transmission method. Latter prevented over-stimulation with clot-formation.

Results: Murine CD133+BMSC co-infused with PRP demonstrated mean adhesion rates of $144 \%$ ( $\pm 17 \%$ StdDev; 
Abstracts: 133rd Congress of the German Society of Surgery (DGCH)

$\mathrm{p}<0.005$ ) along dMEC under flow if contrasted to PPP co-incubation. Pre-stimulation of platelets with ADP demonstrated a further increase leading to improved adhesion of CD133+BMSC compared to unstimulated conditions. Analogue to micro endothelium a boost of mean adhesion rates of CD133+BMSC to $131 \%$ ( $\pm 9 \%$ StdDev; $p<0.05)$ was observed subsequent to unstimulated PRP co-infusion along mSEC.

Conclusion: Our data indicate platelets to trigger adhesion of CD133+BMSC to murine micro vasculature analogue to men in a stimulation independent manner. Further we demonstrate here for the first time that thrombocytes bear the capacity to promote BMSC-adhesion to hepatic vasculature. These data may add to the understanding of mechanisms by which platelets support hepatic generation and offer novel strategies to increase the efficacy of therapeutic BMSC-application in clinical liver disease.

\section{3 \\ Peptide XIB13 Reduces Capillary Leak in a Rodent Burn Model}

L. Von der Lohe ${ }^{1}$, K. Zhuravleva ${ }^{2}$, H. Lauer ${ }^{2}$, M. Lehnhardt ${ }^{2}$, J. Kolbenschlag ${ }^{1}$, K. Schossleitner ${ }^{3}$, P. Petzelbauer ${ }^{3}$,

O. Goertz 1

${ }^{1}$ Martin-Luther Krankenhaus, Berlin, ${ }^{2}$ Bergmannsheil Bochum, Bochum, Germany; ${ }^{3}$ Medical University Vienna, Wien, Austria

Background: Edema due to capillary leak is a generalized and life threatening event in sepsis and major burns for which there is no causal treatment. Local burn wounds are an ideal model to investigate the impact of a new therapeutic agent on edema formation. We aimed to identify peptide sequences of cingulin that can attenuate stress-induced endothelial cytoskeleton disarrangement in vitro and which reduce burninduced edema in vivo.

Materials and Methods: Cingulin-derived peptides were screened in high content cell culture assays monitoring actin displacement and endothelial cell/cell contacts. The ears of male hairless mice were inflicted with full thickness burns using a hot air jet. Mice with and without burn injuries were treated with Xib13 or solvent by continuous intraperitoneal application for 3 days. Edema, microcirculation, leukocyteendothelial interactions and angiogenesis - measured as nonperfused area - were investigated over a 12-day period using intravital fluorescence microscopy.

Results: Xib13 reduced endothelial stress formation and stabilized endothelial tight junctions in cell-cultures. In the burn model, Xib13 improved angiogenesis compared to controls (non-perfused area on day 12: $5.7 \pm 1.5 \%$ vs. $12.0 \pm$ $2.1 \%$; p b 0.05). Edema was significantly reduced at all observation points in Xib13-treated animals as compared to controls (day $12: 67.6 \pm 2.6 \%$ vs. $83.2 \pm 6.4 \%$ ). Furthermore a dose dependent effect could be observed.
Conclusion: Xib13 improved angiogenesis, reduced edema formation. Since edema formation is a serious parameter for burn conversion and is associated with survival it could provide a new treatment option for patients with burn injuries.

4

Horizontal Transfer of microRNA by Bone

Marrow Derived Microparticles Modulates Inflammation after Experimental Liver Resection

M. Schmelzle ${ }^{1}$, K. Splith ${ }^{2}$, I. Kämmerer ${ }^{2}$, C. Hegewald ${ }^{2}$, L. Feldbrügge ${ }^{3}$, J. Schulte am Esch ${ }^{4}$, J. Pratschke ${ }^{1}$,

S. Robson ${ }^{3}$, S. Kuhn ${ }^{2}$

${ }^{1}$ Charité - Universitätsmedizin Berlin, Campus VirchowKlinikum, Berlin, ²Universität Leipzig, Leipzig, Germany;

${ }^{3}$ Beth Israel Deaconess Medical Center, Harvard

University, Boston, USA; ${ }^{4}$ Universitätsklinikum Düsseldorf, Düsseldorf, Germany

Background: Mechanisms involved in the communication between bone marrow stem cells and resident cells in the regenerating liver remain uncertain. We aimed to study implications of horizontal miRNA transfer by bone marrow (BM) derived plasma microparticles (MP) with regard to modulations of vascular inflammation and apoptosis of primary liver cells after partial hepatectomy.

Materials and Methods: Partial hepatectomy was performed in $\mathrm{C} 57 \mathrm{Bl} / 6$ wild type and $\mathrm{Cd} 39$ null mice, with latter being unable to phosphohydrolyze pro-inflammatory extracellular adenosine triphosphate (ATP) to adenosine. MP were isolated from the plasma and cell culture supernatant via two-step ultracentrifugation. Gene and miRNA expression was analyzed using qPCR. For fusion studies, MP were labeled with Cy3-labeled RNA oligonucleotides and stained with the PKH67. Fusion was imaged by FACS and confocal microscopy. Cell viability and apoptosis rate was measured using a commercial apoptosis assay.

Results: After partial hepatectomy, a significant decrease in anti-inflammatory miR-142-3p plasma MP levels was observed in Cd39 null mice ( $p<0.001)$. In vitro stimulation of wild type mononuclear cells (MNC) with anti-inflammatory adenosine significantly boosted miR-142-3p MP levels ( $p<$ 0.01). Those effects were shown to be mediated by extracellular adenosine-type P1 and P2 receptors. Fusion and miR142-3p transfer was noted at significant levels after co-culture of MNC-derived MP abnd HUVEC. Transfection of miR-142-3p significantly reduced pro-inflammatory TNF $\alpha$ mRNA levels $(p<0.01)$ and decreased the amount of apoptotic endothelial cells $(\mathrm{p}<0.05)$.

Conclusion: Bone marrow derived MP are shed after partial hepatectomy into the peripheral blood and contain miR-142-3p in a CD39-dependent manner. Shedded MP fuse and transfer immunomodulatory miR-142-3p, which may 
dampen endothelial inflammation and apoptosis in vivo. Our observations suggest alternative ways of communication between bone marrow cells and primary cells in the liver after partial hepatectomy with important implications in vascular inflammation and liver regeneration.

\section{5 \\ Ileal Interposition in Rats with Experimental Type 2-Like Diabetes (T2LD) Improves Glycemic Control Independently of Glucose Absorption \\ C. Otto ${ }^{1}$, P.R. Rikkala ${ }^{1}$, N. Wagner ${ }^{2}$, I. Vrhovac ${ }^{2}$, I. Sabolic ${ }^{1}$, C.T. Germer ${ }^{1}, H$. Koepsell ${ }^{3}$, C. Jurowich ${ }^{1}$ \\ ${ }^{1}$ Universitätsklinikum Würzburg, Würzburg, Germany; ${ }^{2}$ Institute for Medical Research and Occupational Health, Zagreb, Croatia; ${ }^{3}$ Universität Würzburg, Würzburg, Germany}

Background: Bariatric surgery is the most effective longterm treatment for obesity and improved glucose homeostasis independently of weight loss. In contrast to Roux-Ygastric bypass with partial stomach resection, ileal interposition (IIP) and duodeno-jejunal bypass (DJB) are not thought to change regulatory mechanisms governed by the stomach. Therefore, IIP and DJB are good models for elucidating gutrelated mechanisms to improve glycemic control. We described a down-regulation of Na-D-glucose cotransporter SGLT1, which is rate limiting for intestinal glucose absorption, after DJB leading to increase glucose exposition of L-cells and glucose-dependent stimulation of GLP-1 secretion. This suggests a direct contribution of decreased glucose absorption to the antidiabetic effect of DJB. We elucidated, whether improved glycemic control by IIP based also on SGLT1mediated decrease of glucose absorption.

Materials and Methods: In male Lewis rats $(n=27)$ kept on a high-fat diet, type 2-like diabetes was induced by low-dose streptozotocin $(n=18)$. IIP $(n=9)$ or sham $(n=9)$ operation was performed. The effect of IIP was determined by measuring oral glucose tolerance, insulin tolerance and blood GLP-1 after glucose gavage. SGLT1-mediated glucose uptake in small intestine was measured. In addition, immunostaining for SGLT1 and GLP-1, and electron microscopy were performed.

Results: After IIP, a weight-independent improvement of glucose tolerance, increased insulin sensitivity and increased plasma GLP-1 after glucose gavage was observed. The morphology and function of the interposed ileal segment changed to increased diameter and luminal surface. The number of L-cells increased and the amount of SGLT1mediated glucose uptake in interposed ileum was increased 2 -fold and reached the same level as in jejunum.

Conclusion: Improvement of weight-independent glycemic control following IIP is independent of a change in glucose absorption. The increase of glucose-induced GLP-1 secretion due to increased number of L-cells in the interposed ileal segment and their enhanced exposition to glucose provides a plausible explanation for IIP's antidiabetic effect.

6

Impact of Sleeve Gastrectomy on
Inflammation and Insulin Resistance
in an Obesity Induced Rodent Model

A. Rudeloff, J. Dohmen, H. Matthaei, N. Konieczny,

S. Wehner, J.C. Kalff, P. Lingohr

Universitätsklinikum Bonn, Bonn, Germany

Background: Sleeve gastrectomy (SG) has become an effective surgical treatment for weight reduction in morbid obesity. Additionally, it appears to ameliorate the metabolic situation with respect to obesity induced inflammation and insulin resistance. The metabolic impact of SG and postoperative nutrition are largely elusive and subject of the present study.

Materials and Methods: Male wistar rats ( $=43)$ were fed ad libitum with a high fat diet (HFD) for 15 weeks to induce obesity related inflammation and insulin resistance. Animals underwent SG or sham-operation (SO) followed by HFD or chow diet (CD). The four resulting cohorts were complemented by a reference group of young normal weight rats (CTRL). Weight and fasting blood glucose levels were assessed pre- and postoperatively. Real time qPCR of epididymal adipose tissue was performed to compare the preoperative (HFDpre) and postoperative expression of inflammatory chemokine MCP-1 and adipokines adiponectin and leptin. In addition, epididymal adipose tissue was stained for CD68 to show macrophage infiltration.

Results: After 15 weeks of HFD we observed a significant gain of weight, elevation of blood glucose levels, a higher leptin and MCP-1 expression, a loss of adiponectin expression as well as a distinct macrophage infiltration in HFDpre groups compared to the CTRL group. Postoperative weight was significantly reduced in SG-CD compared to HFDpre rats. Fasting blood glucose levels were lower after SG (SG-CD; SG-HFD) as well as after a sole diet change (SO-CD; SG-CD) compared to HFDpre group. The decline of blood glucose in the SG-CD group was more obvious than in the SG-HFD group. SO-CD and SG-CD animals showed a decrease of leptin expression in comparison to the HFDpre group. Leptin expression reduced comparing SG-HFD with HFDpre rats $(p=0.08)$. When compared to HFDpre group MCP-1 expression decreased significantly in the SO-CD group (67.3\%) and lower in the SG-CD cohort (59.8\%). Due to macrophage infiltration SO-HFD rats exhibited a markedly higher macrophage density compared to rats in the SG-CD group.

Conclusion: Our results demonstrate that SG may have a positive effect on obesity related inflammation and insulin resistance independent from the p.o. diet. The obese 
Abstracts: 133 rd Congress of the German Society of Surgery (DGCH)

phenotype, however, cannot be eliminated through SG alone if animals continue with HFD. SG in combination with CD most effectively reduces the inflammatory response of epididymal adipose tissue. Clinical studies shall prove our findings in order to further optimize the clinical management of patients suffering from morbid obesity.

\section{7 \\ Reflectory Apnea Because of Bleeding after Thyroid Surgery - The Baro-Respiratory Reflex U. Wirth ${ }^{1}$, H.M. Schardey ${ }^{1}$, T. Von Ahnen ${ }^{1}$, U. Pohl', S.K. Schopf \\ ${ }^{1}$ Krankenhaus Agatharied, Hausham, ${ }^{2}$ Ludwig- \\ Maximilians-Universität München, München, Germany}

Background: Postoperative hemorrhage in thyroid surgery is a rare but potentially life-threatening complication. Several different mechanisms leading to asphyxia have been described in literature. In ex-vivo experiments increased cervical pressure seemed unable to cause tracheal compression. Our aim was to investigate the problem more closely.

Materials and Methods: We performed an experimental animal study on German house pigs. Ethics were approved by regional Thuringian government. Experiments were carried out under general anesthesia on ten pigs that had undergone thyroidectomy. The pigs were breathing spontaneously with secured airways. Postoperative hemorrhage was simulated by pumping either blood from the aorta or silicone oil into the surgical site in the neck. Cervical pressure and vital parameters were measured continuously with a focus on respiratory rate.

Results: Increased cervical pressure led to reflex apnea in every animal. There is an evident correlation between increased cervical pressure and initiation of apnea. The apnea occurs irrespective of the kind of anesthesia or pain medication used. A mechanical cause can be excluded. In nine of the ten animals, relief of cervical pressure led to complete respiratory recovery. With complete recovery, we repeated the test up to six times in each pig. Apnea with respiratory recovery was achieved in 23 of 25 tests (92\%). Vital parameters such as heart rate and arterial blood pressure remained stable during the experiments. There was no significant difference between using blood and silicone oil neither to trigger apnea nor in the stability of the vital signs during the tests. The threshold pressure that initiated the decrease of respiratory rate was $46.08 \pm 15.60 \mathrm{~mm} \mathrm{Hg}$, while the cervical compartment pressure needed to induce apnea was $74.72 \pm 24.83 \mathrm{~mm} \mathrm{Hg}$. Blood was used in 18 test procedures and silicone oil in seven.

Conclusion: The mechanism leading to apnea seems to be a pressure-dependent nervous reflex. We present the results of the first in vivo animal experiments on this topic. We describe a completely new mechanism for the asphyxia triggered by increased intracervical pressure and call it the baro- respiratory reflex. Actually we perform further experiments using functional MRI for a better understanding of the involved central nervous areas. Further research will be required to identify the central and peripheral nervous structures and pathways involved.

\section{8 \\ Chemokines (CCL3, CCL4 and CCL5) \\ Efficiently Inhibit ATP-Induced Release of Interleukin-1ß; from Human Monocytic Cells

\author{
A.L. Amati, S. Wilker, W. Padberg, A. Zakrzewicz, \\ V. Grau \\ Universitätsklinikum Gießen und Marburg, Standort \\ Gießen, Gießen, Germany
}

Background: Chemokines and their receptors play a central role in coordinating homeostatic circulation of monocytes as well as their migration to the place of infection or injury. As a response to pathogenic stimuli, recruited mono-

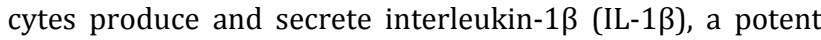
pro-inflammatory cytokine, which plays a central role in host defense. An overproduction of IL-1 $\beta$ can however cause fever, systemic inflammatory response syndrome (SIRS) and even multiorgan damage. Hence, production and release of IL-1 $\beta$ are strictly controlled and dependent on two consecutive danger signals, typically LPS and extracellular ATP, which leads to inflammasome assembly, IL- $1 \beta$ maturation and release. In a recent study from our laboratory, a novel cholinergic pathway was discovered, which controls ATP-induced release of IL-1 $\beta$ from human monocytes. Here, we hypothesize that chemokines are involved in a similar pathway, inhibiting ATP-induced release of IL-1 $\beta$ and discover a mechanism that seems to prevent premature release of the proinflammatory cytokine into the bloodstream.

Materials and Methods: LPS-primed monocytic U937 cells were stimulated with BzATP, a specific ligand of ATP receptor $\mathrm{P} 2 \mathrm{X} 7$, in the presence of increasing concentrations of selected chemokines (CCL3, CCL4, CCL5, CXCL12, and CXCL16). In a similar experimental setting, antagonists of nicotinic acetylcholine-receptors were added to LPS-primed and BzATP-stimulated U937 cells along with CCL3, CCL4 and CCL5. IL-1 $\beta$ released to supernatants was measured by ELISA. To investigate the role of chemokine receptors CCR1, CCR3 and CCR5, U937 cells were transfected with siRNA to these receptors as well as with scrambled control siRNA.

Results: CCL3, CCL4 and CCL5 dose-dependently inhibited BzATP induced release of IL-1 $\beta$ from LPS-primed U937 cells, whereas CXCL12 and CXCL16 were ineffective. Antagonists of nicotinic acetylcholine receptors: mechamylamine, alphabungarotoxin and strychnine, reversed the inhibitory effect of CCL3, CCL4 and CCL5 on BzATP-induced release of IL-1 $\beta$ from LPS-primed U937 cells. In transfection experiments, the inhib- 
Abstracts: 133 rd Congress of the German Society of Surgery (DGCH)

itory effect of CCL3 on IL-1 $\beta$ release was blunted in cells treated with siRNA against CCR1, CCR3 and CCR 5 but not after treatment with control siRNA. The inhibitory effect of CCL4 or CCL5, however, was not impaired.

Conclusion: CCL3, CCL4 and CCL5 effectively inhibit ATPinduced release of IL-1 $\beta$ from LPS-primed U937 cells, whereas CXCL12 and CXCL16 do not influence this pathway. The observed inhibitory effect of the chemokines is mediated via nicotinic acetylcholine receptor subunit alpha9 and chemokine receptors. These results suggest that whenever monocytes are concomitantly stimulated by chemokines and extracellular ATP, inflammasome activation and release of IL-1 $\beta$ into the circulation is inhibited. This mechanism might at the same time prevent SIRS and enhance host defense, as IL- $1 \beta$ could be released to the inflamed tissue upon monocyte transmigration. Further studies are needed to uncover the downstream signaling molecules involved in this pathway and the in vivo relevance of these observations.

\section{9 \\ ATP-Induced Release of IL-1 $\beta$ by Human Monocytes Is Suppressed by Alpha-1 Antitrypsin \\ R. Siebers ${ }^{1}$, S. Wilker ${ }^{1}$, M. Küllmar ${ }^{1}$, K. Richter ${ }^{1}$, J. Klein ${ }^{2}$, \\ N. Aggarwal ${ }^{3}$, A. Zakrzewicz ${ }^{1}$, W. Padberg ${ }^{1}$, \\ S. Janciauskiene ${ }^{3}$, V. Grau ${ }^{1}$ \\ ${ }^{1}$ Justus-Liebig-University Gießen, Gießen, ${ }^{2}$ Goethe \\ University College of Pharmacy, Frankfurt am Main, \\ ${ }^{3}$ Hannover Medical School, Hannover, Germany}

Background: IL-1 $\beta$ is a potent pro-inflammatory cytokine, which plays an essential role in host defense. Excessive release of IL-1 $\beta$ however, can cause life-threatening systemic inflammatory response syndrome (SIRS). In response to diverse danger- or pathogen-associated molecular patterns, an inactive pro-form of this cytokine (pro-IL-1 $\beta$ ) is produced. Normally, a second inflammatory stimulus such as extracellular ATP is needed to induce inflammasome activation, cleavage of pro-IL-1 $\beta$ and release of bioactive IL-1 $\beta$. Recently, we discovered a cholinergic mechanism that inhibits activation of the ATP receptor P2X7 and release of IL-1 $\beta$ by human monocytes (Hecker-A et al. J Immunol 2015). Alpha-1 antitrypsin (AAT) is an important anti-protease of the lung, which, in addition, exerts anti-inflammatory functions via poorly defined pathways. We hypothesize that AAT inhibits ATPinduced release of IL-1 $\beta$ by human monocytes.

Materials and Methods: Primary human monocytes or LPS-primed U937 cells were stimulated with 2'(3')-0-(4-benzoylbenzoyl)adenosine-5'-triphosphate (BzATP) to activate the P2X7 receptor, and IL-1 $\beta$ was measured in cell culture supernatants by ELISA. AAT was applied together with BzATP either alone or in combination with inhibitors of phospholipase A2 (PLA2) or antagonists of the nicotinic acetylcholine receptor (nAChR). SiRNA technique was used to investigate the role of calcium-independent PLA2 (iPLA2) and CD36 in the inhibitory pathway induced by AAT. BzATP-induced ion currents in U937 cells were monitored using patch-clamp technique.

Results: AAT inhibited BzATP-induced release of IL-1 $\beta$ by U937 cells in a dose-dependent manner, but did not show significant effects on ATP-independent mechanisms of inflammasome activation. Inhibition of BzATP-induced IL-1 $\beta$ release was effective in primary human monocytes. Ion currents induced by BzATP in U937 cells were abrogated by AAT. Antagonists of nAChR (mecamylamine, alpha-bungarotoxin and strychnine) as well as a specific inhibitor for iPLA2 (bromoenollactone) reverted the inhibitory effect mediated by AAT. SiRNA technique confirmed participation of iPLA2 and suggested the involvement of CD36 in this inhibitory mechanism.

Conclusion: AAT is a potent inhibitor of ATP-induced IL-1 $\beta$ release by human monocytes. Inhibition involves nAChR, iPLA2 and CD36 and suppresses activation of ATPreceptor P2X7. As polytrauma and major surgery result in excessive release of ATP into the circulation, treatment with AAT might be a promising novel therapeutic approach to prevent SIRS.

\section{0 \\ Monocytes/Macrophages Play a Contributing Role in Lymphangiogenesis Independent of Their Role in Intestinal Inflammation \\ F. Becker ${ }^{1}$, T. Vowinkel ${ }^{1}$, E. Rijcken ${ }^{1}$, I. Tsunoda ${ }^{2}$, \\ D. Ostanin ${ }^{2}$, S. Alexander ${ }^{2}$ \\ ${ }^{1}$ Universitätsklinikum Münster, Münster, Germany; ${ }^{2}$ LSU \\ Health Sciences Center, Shreveport, LA, USA}

Background: Inflammation-associated lymphangiogenesis (IAL) is defined as remodeling within the lymphatic network and growth of new lymphatic vessels during inflammation. Intestinal IAL is observed in inflammatory bowel diseases (IBD) and animal models of colitis and is thought to limit inflammation by maintaining fluid and immune cell clearance from intestinal tissues. IAL is driven mainly by pro-lymphangiogenic vascular endothelial growth factors-C/D released by infiltrated immune cells e.g. monocytes/macrophages. Although monocytes/macrophages have been shown to be associated with the intestinal pathology in IBD descriptively, their roles in intestinal IAL have never been studied mechanistically. Here, we investigated contributions of these cells in development of intestinal inflammation and IAL.

Materials and Methods: To study roles of monocytes/ macrophages in intestinal inflammation and IAL, we used CC-chemokine receptor-2 knockout mice (CCR2-/-), which have reduced levels of circulating monocytes. For induction of chronic colitis, CCR2-/- mice were crossed with recombi- 
Abstracts: 133 rd Congress of the German Society of Surgery (DGCH)

nation activating gene-1-deficient mice (CCR2-/- x Rag-1-/-). To further study the selective contribution of monocytes, bone marrow derived wild type (WT) Ly6Chigh monocytes were isolated and transferred them into colitic WT and CCR2-/- mice. Intestinal inflammation was induced by feeding dextran sodium sulfate (DSS, acute colitis) or by adoptively transferring CD4+CD45RBhigh T-cells (chronic colitis). Tissue-infiltrating cells and intestinal inflammation was assessed by flow cytometry, disease activity and histopathology, while IAL was assessed by lymphatic vessel morphology and density in colon sections immunostained by a lymphatic marker, LYVE-1.

Results: Compared to WT mice, CCR2-/- and CCR2-/- x Rag-1-/- mice showed significantly reduced numbers of infiltrating colon lamina propria Ly6Chigh monocytes. In acute DSS-induced colitis, CCR2-/- mice were protected from intestinal injury, which was accompanied by reduced IAL as measured by a lower lymphatic vessel density and diminished appearance of LYVE-1+ cells. The protective, anti-inflammatory phenotype in colitic CCR2-/- was reversed, when DSS-treated CCR2-/- mice were reconstituted with WT Ly6Chigh monocytes, which resulted in a similar intestinal abundance of LYVE-1+ cells in colitic WT and CCR2-/- mice. In chronic T-cell mediated colitis, CCR2 deficiency did not attenuate the course of inflammation but reduced intestinal IAL.

Conclusion: Our results demonstrate a critical involvement of monocytes/macrophage in acute DSS-induced inflammation and IAL. Conversely, in chronic T-cell mediated colitis, intestinal injury was not attenuated by monocyte reduction, although effects on intestinal IAL persisted. Our data suggest that independent of inflammation per se, monocytes/macrophages play an important contributing role to intestinal IAL. Since restoration of lymphatic function is closely linked with attenuation of inflammation, future IBD therapies might exploit our newly discovered role of monocytes/macrophages to restore lymph clearance and reduce inflammation.

Supported by the German Research Foundation (BE 5619/1-1) and the Department of Defense (PR100451).

\section{1 \\ Contrary Function of Innate Response Activator (IRA) B Cells in Inflammation G. Weber \\ Universitätsklinikum Carl Gustav Carus, TU Dresden, Dresden, Germany}

Background: Innate response activator (IRA) B cells are a subset of B-1a derived B cells that produce the growth factors granulocyte macrophage colony stimulating factor (GM-CSF) and Interleukin-3 (IL-3). In mouse models of sepsis and pneumonia, two majors causes of mortality world wide, B1a B cells residing in serosal sites recognize bacteria, migrate to the spleen or lung, and differentiate to IRA B cells that then contribute to the host response by either amplifying inflammation by the induction of emergency myelopoiesis through the generation of IL-3 or dampening inflammation by the production of polyreactive IgM, a necessary antibody to control especially for gram negative bacteraemia. Here we demonstrate the contrary functions of IRA B cells in various inflammatory models.

Materials and Methods: In our studies we used different genetic, in vivo, ex vivo and in vitro as well as fate mapping and rescue approaches to analyse the immunological function of IRA B cells during inflammation. By using Cecal Ligation and Puncture (CLP), a mouse model of experimental sepsis, we identified the role of IL-3 producing IRA B cells, by using intratracheal and intra-pleural approaches to mimic pneumonia, we identified the role of GM-CSF producing IRA B cells during inflammation.

Results: Our results demonstrate that IRA B cells have contrary functions during acute inflammation, dependent on their production of IL-3 (IRAIL-3 B cells) or GM-CSF (IRAGMCSF B cells). We show that IRAIL-3 B cells are critical mediators for inducing emergency myelopoiesis. IL-3 amplifies the generation of Ly-6Chigh monocytes and neutrophils, and potentiates the cytokine storm during sepsis leading to an increased mortality. In contrary, IRAGM-CSF B cells have protective function during pneumonia, an acute model of local inflammation. By the GM-CSF dependent production of polyreactive $I g M$, our study indicates, the strategically located IRAGM-CSF B cells in the pleural space provide effective early frontline defence against microbes invading the lungs.

Conclusion: Altogether, the work presented here enriches our understanding of immune activation mediated through IRA B cells. The studies identify IRAIL-3 B cells as orchestrators of emergency myelopoiesis and potential therapeutic target for the treatment of sepsis, describe the pleural space as the primary source of IgM in the airways and positions IRAGM-CSF B cells as orchestrators of protective IgM immunity.

\section{2 \\ Deficiency of the Oxygen Sensor HIF-Prolyl Hydroxylase PHD2 Reduces Postoperative Adhesions}

V. Opitz, M.J. Strowitzki, A. Ritter, P. Radhakrishnan,

U. Keppler, J.M. Harnoss, M. Wallwiener, A. Ulrich,

M. Schneider

Universität Heidelberg, Heidelberg, Germany

Background: Over $90 \%$ of patients undergoing abdominal surgery develop intraabdominal adhesions, potentially causing complications such as bowel obstruction or infertility. Hypoxia and necrosis represent major stimuli for adhesion formation. We therefore investigated the significance of the 
key cellular oxygen sensing enzymes, HIF-prolyl hydroxylases (PHD-) 1, -2 and -3 on adhesion formation, and also whether pharmacological inhibition of PHD enzymes is capable of reducing intraabdominal adhesions.

Materials and Methods: Intraabdominal adhesions were induced in PHD-deficient (PHD1-/-, PHD2+/- or PHD3-/-) and wildype (WT) mice applying the ischemic button model. Alternatively, WT mice were pre-treated with the PHD inhibitor EDHB (ethyl-3,4-dihydroxybenzoate) or vehicle (saline) for 3 days prior to induction of adhesions. Quantity and quality of adhesions were assessed seven days thereafter in a blinded fashion applying histological- (e.g. HE, MassonGoldner-Trichrom) and immunohistochemical stainings (e.g. CD45, F4/80, $\alpha$-SMA). qRT-PCR was performed to determine the expression of several pro-fibrogenic and pro-adhesive factors (such as TGF- $\beta$, collagen type 1 and PAI- 1 ).

Results: PHD2-deficient mice showed markedly reduced adhesion-formation compared to WT littermates ( $p=0.01$ ). PHD-inhibition with EDHB likewise significantly reduced adhesion formation. Submesothelial fibrotic tissue fibers were significantly reduced in PHD2-deficient mice, but likewise in animals lacking PHD1 or PHD3 ( $<<0.05)$. Consistently, the length of adhesions was significantly decreased in all three PHD knock out groups ( $p<0.05)$. Of interest, PHD2 deficient mice showed less vascularization of the adhesive fat tissue compared to WT animals ( $p<0.05)$. In addition, the amount of F4/80-positive-cells within the ischemic buttons tissue was significantly reduced in PHD2 deficient mice ( $p=$ 0.02). Within the ischemic buttons EDHB-treated animals showed decreased mRNA expression of VEGF and TGF- $\beta$ compared to untreated animals, albeit these findings did not reach statistically significance.

Conclusion: Pharmacological inhibition of PHD enzymes potentially reduces postoperative adhesion formation, and this effect appears to be preferentially mediated via PHD2. nucleic acids, in particular double stranded RNA (dsRNA) via the TIR-domain-containing adapter-inducing interferon- $\beta$ (TRIF)-TLR-3 pathway is involved in POI.

Materials and Methods: POI was induced by intestinal manipulation (IM) of the small bowel in C57BL/6 (WT) as well as TRIF-/- and TLR-3-/- mice. Pro-inflammatory gene expression was analysed $3 \mathrm{~h}$ after surgery in both groups and compared to untreated mice. POI severity was quantified by expression analysis of inflammatory markers as well as extravasation of MPO+ leukocytes into the ME and gastrointestinal transit (GIT). Additionally, the TLR3 expressing cell population was identified by the generation of bone marrow transplanted mice.

Results: In TRIF-/- mice, gene expression of pro-inflammatory cytokines IL- 6 and IL-1 $\beta$, as well as transcription factor EGR1 were downregulated after IM compared to WT mice. Consequently, TLR3-/- mice demonstrated reduced expression of IL1- $\alpha / \beta$, EGR1 and IFN- $\beta$ during early phase of POI. Type-I interferon associated gene ISG-15 was also decreased in postoperative TLR3-/- mice. In late phase, ME infiltration and POI were ameliorated in both strains reflected by decreased leukocyte numbers and normalized GIT, respectively. WT mice that received TLR-3-/- bone marrow after irradiation were not protected from $\mathrm{POI}$ in contrast to TLR-3-/- mice receiving WT mice bone marrow indicating that resident radio-resistant ME cells are involved in TLR-3 signalling during POI.

Conclusion: In conclusion, our data demonstrate that TLR3 signalling is involved in POI. The TLR3 ligands have yet to be identified but endogenous dsRNA may originate from self RNA formation during the surgical trauma or from luminal viruses, particularly bacteriophages. Perioperative inhibition of this pathway could be a promising target for prevention of POI.

\section{3 \\ Nucleic Acids: The Role of Toll-Like Receptor 3 Signalling in Postoperative Bowel Inflammation}

K. Stein

Universitätsklinikum Bonn, Bonn, Germany

Background: Postoperative ileus (POI) is a common complication after abdominal surgery which is mediated by a complex inflammation of the intestinal muscularis externa (ME). Although bacterial translocation has been described in rodents and patients that underwent abdominal surgery its role in the pathogenesis of POI remained elusive. Previous work of our group demonstrated that toll-like receptor (TLR)-2 and TLR-4, both detecting bacterial cell wall components, were surprisingly not involved in POI development. Herein we tested if recognition of another class of pathogens, 
14

\section{Evaluation of 160 Candiate-Genes Known for Inflammatory Bowel Disease in a Large Cohort of Patients with Diverticulitis}

W. Von Schönfels ${ }^{1}$, B. Buse ${ }^{1}$, S. Buch ${ }^{1}$, J. Tepel ${ }^{2}$, B. Schniewind ${ }^{3}$, I. Vogel ${ }^{4}$, T. Kucharzik ${ }^{3}$, W. Kruis ${ }^{5}$, C. Datz ${ }^{6}$, T. Jacobi ${ }^{7}$, J. Weitz ${ }^{8}$, T. Wedel ${ }^{9}$, T. Becker ${ }^{1}$, A. Gsur ${ }^{10}$, C. Schafmayer ${ }^{1}$

${ }^{1}$ Universitätsklinikum Schleswig-Holstein, Kiel, ${ }^{2}$ Klinikum Osnabrück, Osnabrück, ${ }^{3}$ Klinikum Lüneburg, Lüneburg,

${ }^{4}$ Städtisches Krankenhaus Kiel, Kiel, ${ }^{5}$ Evangelisches Krankenhaus Kalk, Köln, Germany; ${ }^{6}$ Krankenhaus Oberndorf, Oberndorf, Austria; ${ }^{7}$ Diakonieanstalt Dresden, Dresden, ${ }^{8}$ Universitätsklinikum Dresden, Dresden, ${ }^{9}$ Christian-Albrechts-Universität zu Kiel, Kiel,

${ }^{10}$ Medizinische Universität Wien, Wien, Germany

Background: Diverticular disease affects ca. $30 \%$ of adult Europeans over 60 years and causes mortality of 2.5/100,000. Nevertheless, the pathophysiology and genetics are still poorly understood. Even if the heritability is rated between $40-53 \%$, no systematic genetic analysis has been carried out so far.

Many risk factors for diverticular disease have been identified. In particular, a possible association between genetically well explored inflammatory bowel diseases (IBD) and diverticular disease is discussed intensively.

Materials and Methods: Together with our partners in Germany and Austria we could recruit over 10,000 patients with diverticulitis and diverticulosis and about 4,000 healthy controls by means of our recruitment platform POPGEN. 30\% agreed to join the study and sent a completed questionnaire and EDTA-blood for genotyping to the study center. All patients were classified according to the diagnosis in the hospital information system and the completed questionnaire into diverticulitis, diverticulosis and controls and subsequently genotyped with GWAS (genome-wide association study).

In a case-control-study 160 well characterized candidate genes associated with IBD were examined for a possible increased risk for diverticulitis.

Results: In the analyzed cohort none of the genes showed an association with diverticulitis. Only some showed a nonsignificant trend.

Conclusion: In conclusion, we present the first systematic analysis for a genetic risk for diverticulitis and the first population-based cohort for diverticular disease. A common risk profile with IBD could not be found. Further GWAS are needed to determine the genetic risk for diverticular disease.
15

\section{Protein Kinase CK2 Is a Crucial Regulator of Leukocyte-Endothelial Cell Interaction during Ischemia Reperfusion Injury}

\author{
E. Ampofo, D. Widmaier, M. Montenarh, M. Menger, \\ M. Laschke \\ Universität des Saarlandes, Homburg, Germany
}

Background: A hallmark of inflammatory processes such as ischemia and reperfusion $(\mathrm{I} / \mathrm{R})$ is an increased binding of leukocytes to endothelial cells. This interaction requires the expression of specific endothelial surface proteins, which are induced by phospho-regulated signaling pathways. Recently, we found that inhibition of protein kinase CK2 reduces TNF- $\alpha$ induced binding of leukocytes to endothelial cells via downregulation of the endothelial surface proteins E-selectin, intracellular adhesion molecule (ICAM)-1 and vascular cell adhesion molecule (VCAM)-1 in vitro and in vivo. Based on this findings, in the present study, we investigate the function of CK2 during injury caused by I/R.

Materials and Methods: To mimic I/R conditions in vitro, human dermal microvascular endothelial cells (HDMEC) were treated with the CK2 inhibitors TBCA or CX-4945 and subjected to hypoxia/reoxygenation. Afterwards, expression of E-selectin and intercellular adhesion molecule (ICAM)-1 was determined by flow cytometry. Dorsal skinfold chambers were prepared in BALB/c mice, which were pretreated with $75 \mathrm{mg} / \mathrm{kg}$ CX-4945 ( $\mathrm{n}=10)$ or vehicle ( $\mathrm{n}=10)$. Subsequently, by using the model of I/R, the effect of CX-4945 on venular leukocyte adhesion, rolling, velocity and transmigration was analyzed by means of intravital fluorescence microscopy and immunohistochemistry.

Results: We found that TBCA and CX-4945 suppresses the activity of CK2 in HDMEC without affecting their viability. This was associated with a significant down-regulation of E-selectin and ICAM-1 during reoxygenation. In vivo, CX-4945 treatment markedly increased the number of rolling leukocytes and their velocity during reperfusion. In contrast, the number of adherent leukocytes was significantly reduced in CX-4945treated mice $\left(409 \pm 56 \mathrm{~mm}^{-2}\right)$ when compared to vehicletreated controls $\left(610 \pm 78 \mathrm{~mm}^{-2}\right)$. Immunohistochemical analyses further revealed markedly reduced leukocyte transmigration into the ischemic tissue.

Conclusion: These results indicate that CK2 mediates leukocyte-endothelial cell interaction during I/R via regulating the endothelial expression of E-selectin and ICAM-1. Accordingly, CK2 represents a promising target for the treatment of I/R-injury. 
16

\section{Acellular Hypothermic Extracorporeal Perfusion Extends Possible Ischemia Time in a Porcine Whole Limb Replantation Model}

A. Dermietzel' ${ }^{1}$, M. Kückelhaus ${ }^{1}$, M. Alhefzi², M. Aycart ${ }^{2}$, S. Fischer ${ }^{2}$, T. Hirsch ${ }^{1}$, B. Pomahac ${ }^{2}$

${ }^{1}$ Universitätsklinikum Bergmannsheil, Bochum, Germany;

${ }^{2}$ Brigham and Women's Hospital, Boston, USA

Background: Traumatic Amputation is the second leading cause of limb loss in the United States. Amputations only hava a limited time frame available in which successful replantation of an amputated extremity can be performed. This is described as the 'allowable ischemia time'. If an extremity is replanted exceeding this ischemia time the risk of unsuccessful replantation increases exponentially. The patient may be systemically affected by the Ischemia Reperfusion Injury (IRI) resulting in life threatening complications. Extracorporeal perfusion of organs has shown promising results in the preservation of donor organs. In a large animal model we optimized and tested a novel prototype of a portable Extracorporeal Membrane Oxygenator for the artificial perfusion of amputated extremities.

Materials and Methods: Healthy Yorkshire pigs (35-40 kg) were assigned to either control- or perfusion group. The control group consisted of four animals of which the forelimb was preserved for 4 hours on ice slurry, as in a clinical scenario for traumatic amputation. In the perfusion group frontlimbs from three animals were amputated and preserved for 12 hours on the portable perfusion device. Limbs were perfused with a pressure of $30 \mathrm{~mm} \mathrm{Hg}$ at $10^{\circ} \mathrm{C}$ with acellular modified Perfadex ${ }^{\circledR}$-solution. Following the preservation period, forelimbs were replanted and evaluated for ischemia reperfusion injury. The animals were monitored for a total of seven days after replantation.

Results: In six out of seven replantations postoperative monitoring was successfully performed. One control group animal showed signs of Acute Respiratory Distress Syndrome and the experiment was preemptively aborted. Overall, animals from the control group were clinically more affected following replantation than the perfusion group regarding drop in blood-02-Saturation, cardiac fibrillations and a blood parameter change. Most control group animals showed pulmonary impairment and in the aforementioned case, severe pulmonary hemorrhage. With quantitative real time PCR we detected significant differences between the groups for markers of IRI such as Hypoxia Inducible Factor, alpha subunit, Aryl Hydrocarbon Receptor Nuclear Translocator, non-muscle Myosin Heavy Chain 9 and Vascular Endothelial Growth Factor. Histopathological evaluation of muscle biopsies demonstrated greater damage in the control group and electron microscopy confirmed the damage on an ultra structural level.

Conclusion: This study shows the feasibility of ex vivo perfusion of amputated extremities with an acellular, oxygenated perfusion solution in a large animal model.

\section{7 \\ Treatment with Lithium Reduce Ischemia-Reperfusion Injury in Aged and Steatotic Liver in Rats \\ C. Kan}

Universitätsklinikum Jena, Jena, Germany

Background: Lithium has been widely used in the treatment of mental illness. It acts on many stress and survival pathways especially on autophagy pathways. Recent studies showed that treatment with lithium can reduce ischemiareperfusion (I/R) injury in liver via a modulation of MAPK and GSK3b pathways. In this study, we aimed to evaluate the effects of lithium in selective warm I/R model of aged and steatotic liver in rats.

Materials and Methods: Steatosis rats (induced by feeding a high fat and methionine-choline reduced diet for 14 days) and aged rats (2 years) received lithium $(2 \mathrm{mmol} / \mathrm{kg} /$ day, 3 days before and after ischemia). Selective warm ischemia/ reperfusion was induced by clamping the hepatoduodenal ligament of the left lateral and median lobe for $60 \mathrm{~min}$. Animals were observed for $30 \mathrm{~min}, 6 \mathrm{~h}, 24 \mathrm{~h}$ and $48 \mathrm{~h}$ (n =6/group). Read-out parameters consisted of serum liver enzyme levels, HMGB1 translocation and release, liver neutrophil infiltration, MAPK, GSK3b, Caspase 3 and LC3 expression levels.

Results: Treatment with lithium protected against I/R injury in steatotic liver and aged liver, as indicated by lower serum aminotransferase levels, lower inflammatory response (less neutrophil infiltration), lower intracellular stress (less HMGB1 translocation), less apoptosis (lower Caspase 3 expression) and higher levels of autophagy (higher LC3b expression). Treatment with lithium prevented the dephosphorylation of GSK3b and modulated the activation of MAPK pathways after reperfusion.

Conclusion: On the basis of these data, we conclude that treatment with lithium may be a simple way for protecting against I/R injury in steatotic and aged liver. Lithium treatment reduced inflammation and apoptosis via a modulation of MAPK pathway, as well as induced autophagy and reduced necrosis via a modulation of GSK3b pathway.

18

Effects of Remote Ischemic Conditioning in a Rat Model of Orthotopic Liver Transplantation

Z. Czigany ${ }^{1}$, C. Bleilevens ${ }^{1}$, M. Möhring ${ }^{1}$, A. Fulop ${ }^{2}$, A. Szijarto ${ }^{2}$, U.P. Neumann ${ }^{1}$, R. Tolba ${ }^{1}$

${ }^{1}$ Universitätsklinikum Aachen, Aachen, Germany;

${ }^{2}$ Semmelweis Universität, Budapest, Hungary

Background: Orthotopic liver transplantation is a wellestablished and definitive treatment approach for end-stage liver diseases. Nevertheless, ischemic reperfusion injury (IRI) 
Abstracts: 133rd Congress of the German Society of Surgery (DGCH)

of the liver graft is still an important issue in clinical transplantation, especially when we are forced to deal with extended criteria donors. Remote ischemic conditioning (RIC) had already been used successfully by our team to mitigate damage following Pringle maneuver and warm liver IRI.

In the present study we aimed to investigate the effects of remote ischemic conditioning in the recipient using a rat model of arterialized orthotopic liver transplantation.

Materials and Methods: Male Lewis rats were used as donors and recipients $(\sigma \mathrm{n}=144$; median bodyweight: $278 \mathrm{~g}$, range: $240-340 \mathrm{~g}$ ). Surgical procedures were performed in isoflurane anesthesia. Donor livers were flushed with and stored in $4^{\circ} \mathrm{C}$ Histidine-tryptophan-ketoglutarate solution for 8 hours and implanted into recipient animals. Recipients were randomly allocated into three experimental groups: RIC1, RIC2, Control ( $n=24$ /group). In RIC1 and RIC2 groups conditioning was applied in the recipient animal as 4 cycles of $5 \mathrm{~min}$ of ischemia and $5 \mathrm{~min}$ of reperfusion ( $40 \mathrm{~min}$ ) by clamping the infrarenal aorta before liver exclusion or after reperfusion, respectively. After 1, 3, 24, and 168 hours of portal reperfusion, intraoperative measurements (portal vein flow, liver microcirculation) were performed and subsequently animals were sacrificed in deep anesthesia ( $n=6 /$ group/time point). Graft injury was evaluated using various methods.

Results: RIC1 resulted in significantly $(p<0.05)$ improved portal venous flow (PVF, mean \pm SD, RIC1 $24 \mathrm{~h}$ vs. Control $24 \mathrm{~h}$, $22 \pm 2.9$ vs. $16.8 \pm 4.3 \mathrm{~mL} / \mathrm{min}$ ) and microcirculation (Microcirculation, RIC1 $1 \mathrm{~h}$ vs. Control $1 \mathrm{~h}, 105 \pm 13$ vs. $70 \pm 17$ Flow/ Velocity, RIC1 1 h vs. Control 1 h, $19 \pm 1.2$ vs. $14 \pm 0.4$ Arbitrary Units). After $24 \mathrm{~h}$ of reperfusion, the application of RIC has significantly reduced tissue injury compared to the Control group according to the serum levels of ALT, AST, LDH and result of histological analysis. In parallel, strongly reduced terminal deoxynucleotidyl-transferase dUTP nick end labeling (TUNEL) staining was detected on the slides of the RIC1 and RIC2 groups after $3 \mathrm{~h}$ of reperfusion (TUNEL, RIC1 vs. RIC2 vs. Control, $4.3 \pm 1.1$ vs. $3.2 \pm 0.8$ vs. $14.2 \pm 1.7$ apoptotic cells/ high power field; RIC1 and RIC2 vs. Control $\mathrm{p}<0.001$ ). There was an obvious positive tendency concerning the tissue adenosine-triphosphate (ATP) levels between the RIC and Control groups throughout the entire observation period, however, the difference reached the level of statistical significance exclusively after 3 hours of reperfusion (ATP, RIC2 $3 \mathrm{~h}$ vs. Control $3 \mathrm{~h} \mathrm{p}=0.002$ ). In the RT-PCR analyses, a prominent up-regulation was observed in the tissue hemoxygenase-1 (HO-1) expression after 3 hours of reperfusion ( $p<0.001$ vs. normal expression in all groups; RIC1 vs. Control $p=0.04$, RIC2 vs. Control $p=0.83$ ). Supporting findings were obtained from the measurement of serum cytokine (IL-10, MCP-1) and tissue malondialdehyde levels.

Conclusion: Our results suggest that remote ischemic conditioning in the recipient can protect liver grafts in a rat model of arterialized orthotopic liver transplantation. Although, slight differences were found between RIC1 and RIC2 protocols, the optimal timing for the procedure needs to be further elucidated.

\section{9}

\section{Extracorporeal Membrane Oxygenation Prior to Lung Transplantation Alters Long-Term T-Cell Responses}

N. Strobl', H. Winter ${ }^{1}$, G. Warnecke ${ }^{2}$, E. Nößner ${ }^{3}$, A.K. Knöfel ${ }^{2}$, M. Svihla ${ }^{1}$, R. Schramm ${ }^{1}$, R. Hatz ${ }^{1}$, A. Haverich ${ }^{2}$, G. Preissler ${ }^{1}$

${ }^{1}$ Klinikum der Universität München, LMU, München,

${ }^{2}$ Medizinische Hochschule Hannover, Hannover, ${ }^{3}$ Helmholtz Zentrum München, Deutsches

Forschungszentrum für Gesundheit und Umwelt, München, Germany

Background: Extracorporeal membrane oxygenation (ECMO) may be necessary in selected patients with terminal respiratory failure to bridge the time to lung transplantation (LTX). Nevertheless, ECMO represents a maximally invasive therapy, associated with strong systemic inflammatory reactions. However, immunological consequences of perioperative ECMO-therapy in LTX have not been evaluated, so far. Therefore, systemic T-cell responses were analyzed prospectively after LTX in recipients supported by ECMO.

Materials and Methods: In total, 47 (m/f: 21/26, age: $54 \pm$ $2 \mathrm{y}$, LAS: $46 \pm 2)$ patients undergoing single $(\mathrm{n}=20)$ or bilateral $(\mathrm{n}=27)$ LTX due to idiopathic fibrosis $(\mathrm{n}=28)$, cystic fibrosis $(\mathrm{n}=3)$, COPD $(\mathrm{n}=12)$, primary pulmonary hypertension $(\mathrm{n}=$ 1), sarcoidosis ( $n=1)$ and alpha- 1 antitrypsin deficiency ( $n=$ 2) in the period from September/2013 to May/2015 were included in the study, respectively. Five patients (age: $43 \pm 8 y$, LAS: $76 \pm 10$ ) received ECMO-therapy in combination with mechanical ventilation before (ECMO: $634 \pm 312 \mathrm{~h}$; ventilation time (VT): $446 \pm 235 \mathrm{~h}$ ) and during/after LTX (ECMO: $120 \pm$ $67 \mathrm{~h}$; VT: $706 \pm 252 \mathrm{~h}$ ), nine patients (age: $49 \pm 5$ y, LAS: $54 \pm$ 6) were supported by ECMO-therapy following LTX (ECMO: $224 \pm 124$ h; VT: $733 \pm 253$ h), while 33 recipients (age: $58 \pm$ $1 \mathrm{y}$, LAS: $39 \pm 1$ ) were transplanted without ECMO (VT: $240 \pm$ $75 \mathrm{~h}$ ), respectively. Samples for blood count, CRP and flow cytometry analysis of helper (Th: CD3+/CD4+ ; Th1: CD3+/ CD4+/IFN- $\gamma+$; Th2:CD3+/CD4+/IL-4+), cytotoxic (CTL: $\mathrm{CD} 3+/ \mathrm{CD} 8+$ ), and regulatory $\mathrm{T}$-cells (Treg: CD3+/CD4+/ CD25+/FoxP3+), were retrieved prior to LTX (day 0) and 7, $14,21,90,180$ and 365 days after LTX, respectively. Lung function tests were done in the same intervals, postoperatively. Mean follow up time was $264 \pm 17$ days. Data are given as mean \pm SEM.

Results: Leukocyte counts and CRP-values were not significantly different between the groups. However, following ECMO-bridging the CD4+/CD8+-ratio was significantly reduced to $0.9 \pm 0.3$ already on day 0 when compared to non-ECMO patients $(3.1 \pm 0.6)$, remained significantly suppressed until day 90 and showed the same trend as long as one year after LTX (22-53\% reduced). This was accompanied by an increase of the Th1/Th2-ratio. Interestingly, CD4+/ CD8+-ratio in patients treated with ECMO during/after LTX 
Abstracts: 133 rd Congress of the German Society of Surgery (DGCH)

was not different from non-ECMO patients. Although statistically not significant, Tregs in bridged patients were reduced by $27-82 \%$ from day 7 to day 180 after LTX when compared to non-ECMO patients. In contrast, Treg frequency in patients treated with ECMO during/after LTX was similar to that of non-ECMO patients. There was no statistically significant difference in the FEV1 after LTX. Treated acute rejection episodes were noted in one patient after ECMO-bridging (20\%).

Conclusion: ECMO-therapy as bridge to transplant represents a significant trigger for an altered T-cell response, detectable at least as long as one year after LTX. Although the explanatory power of our study is limited by the small sample size, the observed shift of the CD4+/CD8+-ratio in conjunction with reduced Tregs and a higher proportion of rejections might be indicative for an augmented immune response. Interestingly, ECMO therapy during and after LTX did not induce the same changes, which may be related to the immunosuppressive therapy starting simultaneously with the establishment of the extracorporeal circuit.

\section{0 \\ Correlation of Donor Derived Cell-Free DNA and Donor-Specific HLA-Antibodies with Clinical Outcomes after Liver Transplantation}

J. Bucher, M.O. Guba, J. Werner, T. Kauke

Klinikum der Universität München, München, Germany

Background: The immunological and clinical consequences of circulating donor-derived DNA (dd-DNA) and donor-specific HLA-antibodies (DSA) after liver-transplantation are unclear. Dd-DNA may serve as an early graft integrity marker but may also have immune-regulatory property. DSA indicate humoral immune response and may be harmful for the liver graft.

Materials and Methods: We analyzed dd-DNA and DSA in a random sample of 63 liver transplant recipients between 2009 and 2012. Dd-DNA was measured by quantitative PCR, DSA by Luminex Single Antigen Bead Assay at day 3, week 3 and month 3 post-transplant. Both, dd-DNA and DSA were correlated with 1 y graft survival, rejection rates and complications of the large (anastomotic strictures (AS)) and small bile ducts (ITBL).

Results: Dd-DNA was found in $98 \%$ of the patients at day 3 after transplantation. In all patients the amount of dd-DNA decreased until month 3. Forty-nine percent of patients showed a macrochimerism, defined as more than $1 \%$ of dd-DNA. Alloantibodies were found in 27 of 63 (42\%) patients. The vast majority (81\%) of alloantibodies were donor specific (DSA), directed against HLA-class II (87\%). DSA were associated with the development of ITBL (29\% vs. $8 \%, p=0.03$ ) but not with AS. High levels of dd-DNA inversely correlated with biliary complications (ITBL $6 \%$ vs. $37.5 \%$ and AS $9.6 \%$ vs. $37.5 \%, p=0.01$ ). Both, DSA and ddDNA did not correlate with 1 y graft survival or rejection episodes, but interestingly, low levels of dd-DNA were associated with the development of DSA ( $72 \%$ vs. 39\%, p = 0.01).

Conclusion: Our first results suggest that evolution of DSA is dependent of the release of ddDNA. DSA may play a causal role for small bilary complications while dd-DNA seems to be protective.

\section{1}

\section{Gene Expression Measurements for the Non-Invasive Diagnosis of Acute Rejection in Kidney Graft Recipients}

P. Gombos, C. Sommerer, M. Mieth, P. Schemmer, M. Zeier, C. Süsal

University of Heidelberg, Heidelberg, Germany

Background: If not recognized and treated timely, acute rejection episodes (ARE) in the early posttransplant phase can have a negative impact on long-term kidney graft survival. Since the diagnosis of early rejection is still based primarily on the invasive biopsy method, the invention of non-invasive laboratory parameters for diagnosis and prediction of ARE is an urgent need. In the present study we investigated whether the measurement in peripheral blood of transcripts of Foxp3 and CCR7, which are associated with tolerance, and transcripts of PSEN1, which has been associated with acute rejection, may serve as a non-invasive tool to predict kidney allograft rejection in its earliest stages before damage occurred in the transplant.

Materials and Methods: Gene transcripts were measured by Real-Time PCR in 1,486 serially obtained peripheral blood samples of 419 kidney graft recipients between 2001 and 2012. Based on histopathological diagnosis, recipients were classified into non-rejectors (Non- $\mathrm{n}, \mathrm{n}=246$ ), borderline rejectors (Bord- $R, n=78$ ), patients with acute tubular necrosis (ATN, $n=66$ ) and acute rejectors (AR, $n=29)$. Blood samples were collected on days $0,5-7,8-10,11-13$ and $14-16$ after transplantation. Day 1-4 samples served as individual calibrator.

Results: Already on posttransplant days 5-7, CCR7 transcripts were significantly lower in the AR group than in the Non-R group ( $\mathrm{p}=0.028$ ). Foxp3 expression was lower and PSEN1 expression was higher in the AR group than in the Non-R group, however, the difference did not reach statistical significance. Bord-R and ATN patients showed intermediately high values. At this early time point, PSEN1/Foxp3-ratio was significantly higher in the AR group than in the Non-R group $(\mathrm{p}=0.030)$. Although higher in AR patients than in Non-R patients, the PSEN1/CCR7-ratio did not show a significant difference.

On posttransplant days 11-13, AR patients demonstrated strikingly lower Foxp3 and CCR7 gene transcripts ( $p=0.002$ and $p=0.004$, respectively) and significantly higher PSEN1 
gene expression $(\mathrm{p}=0.045)$ than the Non- $\mathrm{R}$ group. At this same sampling day, the PSEN1/CCR7-ratio was also significantly higher in AR patients than in Non-R patients $(\mathrm{p}=0.014)$, whereas the PSEN1/Foxp3 ratio revealed no significant difference.

Conclusion: Our data suggest that gene expression measurement in peripheral blood could serve as a useful noninvasive tool for the detection of graft rejection in its earliest stages.

\section{2}

\section{Retracted}

\section{3 \\ Graft Maturation after Repopulation of Decellularized Rat Livers}

B. Struecker ${ }^{1}$, A. Butter ${ }^{1}$, K. Aliyev ${ }^{2}$, K. Hillebrandt ${ }^{1}$, N. Raschzok ${ }^{1}$, A. Selke ${ }^{1}$, J. Pratschke ${ }^{1}$, I. Sauer ${ }^{1}$

${ }^{1}$ Charité - Universitätsmedizin Berlin, Berlin,

${ }^{2}$ Universitätsmedizin der Johannes Gutenberg-Universität Mainz, Mainz, Germany

Background: Decellularization of livers of different species is a well-established procedure but data on liver recellularization and implantation is limited to proof-of-conceptstudies. Information on the functional evolution and re-organization of grafts after repopulation and during ex vivo maturation is not available.

Materials and Methods: We established a novel proprietary bioreactor to repopulate decellularized rat livers ( $\mathrm{n}=$ 21) with primary rat hepatocytes via the hepatic artery in order to evaluate graft morphology and function during seven days of ex vivo perfusion via the portal vein. Grafts were analyzed at $1 \mathrm{~h}, 6 \mathrm{~h}, 12 \mathrm{~h}, 24 \mathrm{~h}, 3 \mathrm{~d}, 5 \mathrm{~d}$ and $7 \mathrm{~d}$ (all $\mathrm{n}=3$, respectively) by immuno-histologic evaluation, measurement of hepatocyte-related enzymes (AST, ALT, LDH) and albumin in the perfusate, and expression of hepatic metabolism genes (albumin, Cyp1A2).

Results: We present the first available protocol for repopulation of rat livers via the hepatic artery. Within the first 24 hours after repopulation hepatocytes seem to migrate out of the vascular network and build cluster in the parenchymal space around vessels. Graft function is increasing until 24 hours after repopulation and then constantly decreases, although hepatocytes were viable even after seven days of perfusion.

Conclusion: Our data suggests that ex vivo maturation should be held as short as possible and repopulated grafts should be implanted as soon as cell engraftment and graft re-organization is completed.

\section{4 \\ Ensheathing Cells Co-Cultured with Human NT2 Neurons in vitro on 3D Spider Silk Constructs Documented with Time Lapse Imaging}

D. Schröder

Medizinische Hochschule Hannover, Hannover, Germany

Background: The development of effective treatments for spinal cord injury is of considerable medical interest. Transplantation of myelin-forming cells such olfactory ensheathing cells (OECs) and Schwann cells (SCs) into traumatic spinal cord injuries can significantly improve functional outcome in experimental models. In contrast to peripheral nerve system the regeneration of axons from the spinal cord is very limited. The reasons are complex and it is assumed that intrinsic repair might be supported by providing additional cell transplantation combined with an ideally structured microenvironment at the site of injury. Spider silk is a proteinaceous fibre with low immunogenicity and high support of cell migration and adhesion. In the proposed study we determined the in vitro characteristics of ensheathing cells (SCs and OECs) co-cultured with human NT2 neurons seeded on spider silk.

Materials and Methods: Native spider silk from the golden web spider 'Nephila edulis' was harvested and transferred onto frames to provide a three-dimensional structure for the co-culture. NT2 neurons were seeded onto the spider silk constructs, followed by 10 -fold number of OECs and SCs. To

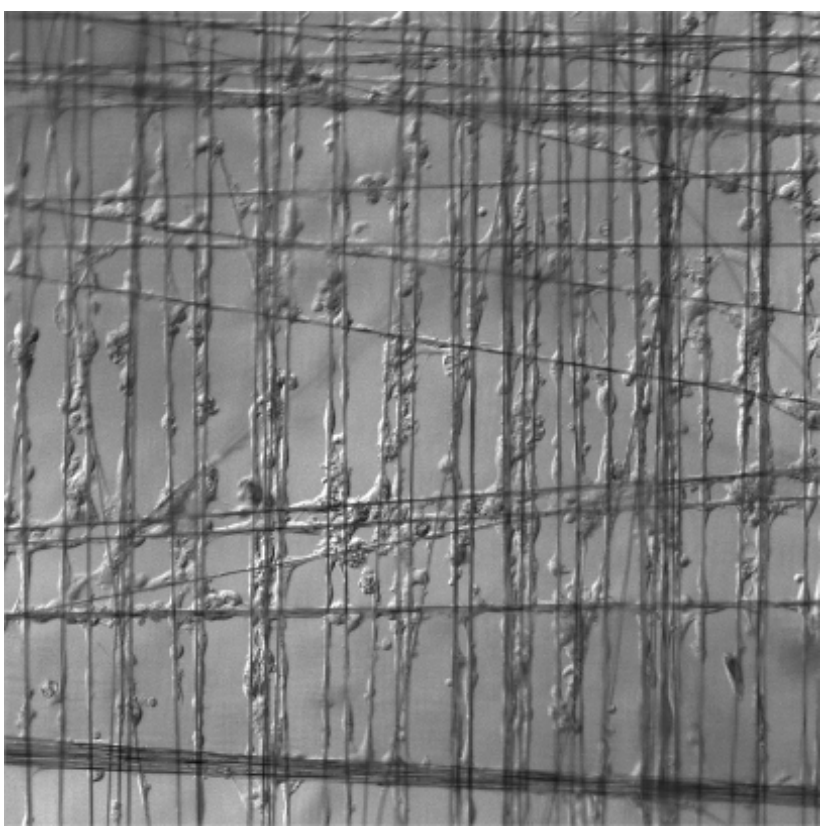

Fig. 1. Co-cultured NT2 neurons with ensheathing cells on spider silk, 10-fold, phase (for Abstract 24). 
Abstracts: 133rd Congress of the German Society of Surgery (DGCH)

analyse the viability and migration of the seeded OECs on the silk time lapse recordings with a Live Cell Imaging System were photographically documented. Furthermore high resolution immunofluorescence images were visualised to elucidate the interactive networking between ensheathing cells, neurons and silk fibres.

Results: The immunofluorescence images demonstrate high numbers of viable cells which moreover express their characteristics. The time lapse recordings show that the cells not only survive, but interactand align on the silk. Furthermore, extensive migration of the ensheathing cells could be observed on the spider silk fibres, as well as formation of long filopodia.

Conclusion: Ensheathing cells co-cultured with NT2 neurons seeded onto spider silk might have considerable advantages concerning transplantation in injured spinal cords. The co-culture model of neurons, ensheathing cells and guiding construct holds promising potential regarding neural regrowth within the CNS and application as a medical research tool.

\section{5}

\section{Custom Made Fibrin Glue Nerve Conduits Optimize the Repair of Peripheral Nerves in a Sciatic Nerve Graft Model in Rats}

J.M. Mayer, C. Krug, T. Holzbach, R. Giunta

Chirurgische Klinik LMU München Innenstadt, München, Germany

Background: Incomplete nerve regeneration after injuries to the peripheral nervous system remains a significant problem in clinical routine and raises the need for supportive strategies. In this study we examined the effect of an additional fibrin glue conduit in the model of a peripheral nerve defect in the rat treated with an autologous nerve transplant.

Materials and Methods: In this study we examined the effect of a custom made fibrin glue conduit as additional nerve guide for an autologous nerve autograft in a $20 \mathrm{~mm}$ sciatic nerve defect model. Here a $20 \mathrm{~mm}$ segment was resected in the course of the right sciatic nerve of the rat $(n=18)$ and reversely coapted under the microscope in terms of an autologous interposition. In the experimental group $(n=9)$ the autograft was additionally incased in nerve guide made of two-compound fibrin glue (ARTISS ${ }^{\circledR}$; Fa. Baxter) measuring $25 \mathrm{~mm}$ in length, with a 2 -mm lumen and $2-\mathrm{mm}$ wall thickness covering both coaptation sites.

During the trial period of 16 weeks we conducted walkingtrack and static foot-print-analyses weekly. After 16 weeks the gastrocnemius muscle was weighed. Morphometric evaluation including histology, axon counts and measurement of axonal thickness was carried out 16 weeks post-op.

Results: Functional analysis in terms of SFI (sciatic function index) showed significant differences between the groups $(\mathrm{p}<0.05)$ indicating faster functional regeneration in the fibrin glue conduit group.

The gastrocnemius muscle\&rsquo;s weight was significantly increased ( $68 \%$ vs. $54 \%$ of contralateral side; $p<0.05$ ).

Conduit-treated animals showed a higher fraction of large axons, while at the same time presenting lower levels of nerve tissue fibrosis in all segments of the operated side. The proportion of large axons as a fraction of the total number of axons was significantly higher in the distal $1 / 3$ of the nerve grafts $(p<0.05)$ in the conduit group compared to controls. Correspondingly the fraction of small axons was significantly higher in the distal half of nerve grafts in control group.

Conclusion: These results indicate the beneficial effect of a custom made fibrin glue nerve guide in addition to microsurgical nerve repair in the model of an autologous nerve transplant in the rat, yet the underlying mechanisms need to be further investigated.

\section{6 \\ The Inhibition of GDF8 (Myostatin) Rescues Diabetes Associated Bone Healing Deficiencies \\ C. Wallner, S. Abraham, M. Wagner, K. Harati, H. Zöllner, M. Lehnhardt, B. Behr}

Bergmannsheil Bochum, Bochum, Germany

Background: Bone tissue features a reliable healing capacity. However certain pathologies such as diabetes mellitus cause bone healing deficiencies. The epidemiological impact of diabetes mellitus combined with the poor treatment options of associated bone healing impairment demand research of adequate therapeutic methods. In previous studies, we have shown a significant reduction of bone regenerative and angiogenesis in diabetic mice. Recent studies suggest a highly underestimated importance of GDF8 (Myostatin) - an essential protein of muscle catabolism - in bone metabolism.

The goal of this study is to investigate the role of GDF8 in diabetic bone healing. As a next step we seekd to utilize GDF8 inhibitors (Follistatin) to enhance bone healing in diabetic bones.

Materials and Methods: We used an established murine tibial defect model in diabetic (Leprdb-/-) and wildtype mice. Uninjured tissue from both genotypes was processed to evaluate the gene expression of GDF8.

Subsequently, GDF8-Inhibitor Follistatin was administered in tibial bony defects of diabetic mice. Through histology, immunohistochemistry and QRT-PCR angiogenesis, osteogenesis, differentiation and proliferation were analysed.

Results: We have shown a significantly higher expression of GDF8 in diabetic bone compared to wildtype. Application of GDF8-inhibitor showed a significant improvement in diabetic bone regeneration compared to the control group (diabetic animals without treatment). Immunohistochemistry revealed a significant higher proliferation, angiogenesis and osteogenic 
differentiation in Follistatin treated diabetic animals as compared to controls.

Conclusion: GDF8, known for the importance in muscle diseases, seems to play an important role in bone metabolism. In diabetes mellitus bony tissue showed an overexpression of this catabolic protein. Antagonization of GDF8 in diabetic animals leads to a complete restoration of the impaired bone regeneration and represents a promising therapeutic option.

\section{7 \\ Osteoporotic Metaphyseal Fracture Healing Under Extracorporeal Shock Wave Therapy in a Rat Model \\ G.A. Mackert ${ }^{1}$, L. Harhaus ${ }^{1}$, M. Schulte ${ }^{1}$, C. Hirche ${ }^{1}$, D. Kotsougiani ${ }^{1}$, J. Vogelpohl', M. Lehnhardt ${ }^{2}$, U. Kneser ${ }^{1}$ \\ ${ }^{1}$ Berufsgenossenschaftliche Unfallklinik \\ Ludwigshafen, Universität Heidelberg, Ludwigshafen, \\ ${ }^{2}$ Berufsgenossenschaftliches Universitätsklinikum \\ Bergmannsheil, Bochum, Germany}

Background: Osteoporosis and osteoporotic fractures are becoming an increasing burden on the health care system, resulting in longer hospital and rehabilitation-facility stays as well as in increased mortality and morbidity rates in the ageing patient population. This study evaluates the effect of Extracorporeal Shock Wave Therapy (ESWT) on the speed and quality of osteoporotic fracture healing in a rat model.

Materials and Methods: 144 three-month old female Sprague-Dawley rats were used, of which 132 developed manifest osteoporosis 8 weeks after ovariectomy. 12 rats were sham-operated (non-osteoporotic control group: SHAM). The rats received bilateral, metaphyseal tibia-osteotomies and fixation with a mini T-plate. 11 groups were formed. 2 Groups, the SHAM and the osteoporotic control group, received no ESWT. 9 groups received different combinations of ESWTintensities $\left(0.15 \mathrm{~mJ} / \mathrm{mm}^{2}, 0.35 \mathrm{~mJ} / \mathrm{mm}^{2}\right.$, or $\left.0.55 \mathrm{~mJ} / \mathrm{mm}^{2}\right)$ and application-frequencies (day 1 or $1 / 11 / 26$, or $1 / 8 / 15 / 22 / 29$ ) during a 35-day healing period. On day 35 , the tibiae were harvested. They underwent qualitative testing by a 3-point bending-and breaking test (determining stiffness (S), yield load (yL), maximum force (Fmax), and failure load (fL) of the newly formed callus) as well as quantitative and dynamic evaluation through high-resolution micro-CT imaging, histomorphometric analyses and immunofluorescence labeling. Geneexpression was investigated via PCR for the transcripts of osteocalcin, IGF-1, Collagen 1- $\alpha$, Estrogen-Receptor- $\alpha$, and tartrate-resistant acid phosphatase.

Results: Overall, there was a significant improvement in the osteoporotic fracture-healing under ESWT compared to the osteoporotic animals who did not receive ESWT treatment and the non-osteoporotic SHAM animals. The biomechanical analyses showed higher average results for Stiffness (S), yield load (yL), maximum force (Fmax), and failure Load (fL) for those treated with ESWT. The application of $3 \times 0.15 \mathrm{~mJ} / \mathrm{mm}^{2}$ was superior to more intensive treatment groups. Thus, the average $\mathrm{S}$, Fmax and fL was more significant with $\mathrm{S}=96 \mathrm{~N} /$ $\mathrm{mm}, \mathrm{Fmax}=41 \mathrm{~N}$, and $\mathrm{yL}=37 \mathrm{~N}$ compared to the SHAM $(54 \mathrm{~N} /$ $\mathrm{mm}, 33 \mathrm{~N}$ and $28 \mathrm{~N}$ ) and the osteoporotic control group (50 N/ $\mathrm{mm}, 40 \mathrm{~N}$ and $33 \mathrm{~N}$ ) respectively. There were similar trends up to the groups 9-11, where the intensity was higher, but the values for S, yL, Fmax and fL were lower, narrowing down the range of the beneficial therapeutic intensity level. PCR investigations showed an increased transcription of osteo-anabole and osteo-catabole gene-products, implying an overall increased bone turnover in those groups treated with ESWT compared to the osteoporotic, non-ESWT treated groups. The micro-CT analyses and the histomorphometric investigations were in line with these results showing significantly more callus formation in the earlier phases under ESWT-treatment.

Conclusion: In conclusion, in this study we found that ESWT can improve osteoporotic fracture healing qualitatively and quantitatively. This study simultaneously enabled us to find the best ranges of intensities and application-frequencies for optimal fracture healing under ESWT. And with rat boneproperties and metabolism being very similar to that of humans, applying this to human bone could mean a tremendous improvement in the therapeutic strategies of osteoporotic human fracture healing, as well as an improvement in patient health and a significant reduction of health care system costs.

\section{8}

\section{A Novel Tool for Continuous Fracture Aftercare - Clinical Feasibility and First Results of a New Telemetric Gait Analysis Insole}

B.J. Braun, N.T. Veith, M. Rollmann, J. Holstein,

T. Pohlemann

Universitätsklinikum des Saarlandes, Homburg, Germany

Background: Weight bearing after lower extremity fractures remains a highly controversial issue. Even in ankle fractures, the most common lower extremity fracture no standard aftercare protocol has been established. Average non-weightbearing times range from 0-7 weeks, with standardized, radiological healing controls at fixed time intervals (Swart, Injury 2015). Recent literature calls for patient-adapted aftercare protocols based on individual fracture and load scenarios (Smeeing, PloS one 2015).

We show the feasibility and first clinical results of a new, insole embedded gait analysis tool for continuous, individual monitoring of gait, load and activity after lower extremity fractures.

Materials and Methods: In a prospective, observational study 14 patients with ankle fractures (Weber-B type, no syndesmotic injury) were continuously monitored with a new, independent gait analysis insole for up to 3 months post- 
Abstracts: 133 rd Congress of the German Society of Surgery (DGCH)

operatively. Strict $20 \mathrm{~kg}$ partial weight bearing was ordered for 6 weeks. Overall activity, load spectrum, ground reaction forces, clinical scoring (VAS pain, Olerud Molander Score, AOFAS score, EQ5d) and general health data were recorded and correlated. Statistical analysis with power analysis, t-test and Spearman correlation was performed.

Results: Based on the parameters load, activity, gait time over $20 \mathrm{~kg}$ weight bearing and maximum ground reaction force high and low performers were defined after 3 weeks. At the time of definition no significant difference between VAS pain, Olerud-Molander Score and AOFAS score was seen between both groups $(\mathrm{p}>0.22)$. The previously defined high performers reached time to full weight bearing after 6 weeks significantly earlier than the low performers $(3.3 \pm 4.1$ vs. $13.3 \pm 5.9$ days; $p=0.01$ ). Strong correlation was seen between VAS pain ( $\mathrm{rs}=-0.95 ; \mathrm{p}=0.001$ ), Olerud-Molander Score (rs = $0.93 ; p=0.004)$ and the AOFAS Score ( $r s=0.74 ; p=0.003$ ) and the ability to bear weight over 3 months. The patients significantly underestimated their weight-bearing time over the prescribed limit (49.9 \pm 52.2 vs. $188.3 \pm 190.6$ minutes; $\mathrm{p}<$ 0.01 ). The weight-bearing limit was upheld by only one patient. Average time over the limit was 572.4 minutes during the first 6 weeks after surgery.

Conclusion: Early, continuous gait analysis is able to define aftercare performers with significant differences in time to full painless weight bearing where clinical or radiographic controls could not. Patient compliance to standardized weight bearing limits and protocols is low. Highly individual rehabilitation patterns were seen in all patients. Aftercare protocols should be adjusted to real-time patient conditions, rather than fixed intervals and limits. With a real-time measuring device patient performance levels could be identified early and influenced towards optimal healing conditions.

With the successful application of this new aftercare tool further studies with large patient numbers are now possible to extend the aftercare principle to further lower extremity fracture situations.

\section{9 \\ Clinical and Prognostic Relevance of the 12-Gene Signature Associated with Genomic Instability in Breast Cancer \\ N. Doberstein ${ }^{1}$, M. Oberländer ${ }^{1}$, H. Alkemade ${ }^{1}$, F. Ernst $^{1}$, S. Freitag-Wolf ${ }^{2}$, C. Thorns ${ }^{3}$, T. Gemoll ${ }^{1}$, J.K. Habermann ${ }^{1}$ \\ ${ }^{1}$ Universitätsklinikum Schleswig-Holstein \\ Campus Lübeck, Klinik für Chirurgie, Lübeck, \\ ${ }^{2}$ Universitätsklinikum Schleswig-Holstein, Campus Kiel, \\ Kiel, ${ }^{3}$ Universitätsklinikum Schleswig-Holstein, Campus \\ Lübeck, Lübeck, Germany}

Background: To avoid over- or undertreatment and to reduce toxic effects of chemotherapy for breast cancer patients, it is of high impact to make an accurate diagnosis and estimation of prognosis. The previously identified 12-gene signature by Habermann et al. (2009) distinguishes between genomically stable and unstable breast carcinomas and thus estimates the respective prognosis. The objective of this study was to validate the 12-gene signature's prognistic relevance at the protein level by immunohistochemistry and association with clinical parameters.

Materials and Methods: The protein expression was semiquantitatively determined by immunohistochemical staining of a tissue microarray consisting of 405 clinical formalin-fixed and paraffin embedded (FFPE) tissue samples (carcinoma, DCIS, benign neoplasia and normal tissue) from 245 patients. The samples were scored by a senior pathologist according to the percentage of the positively stained cells (score $0:<1 \%$, score $1: \leq 20 \%$, score $2: \leq 50 \%$, score 3 : $>50 \%$ positive cells). Subsequently, statistical analyses were performed to evaluate the associations for correlations with clinical parameters and patients\&rsquo; survival.

Results: A total of eight target proteins was successfully hybridized and evaluated: AURKA, CDKN2A, FOXA1, NXF1, ACADSB, PSMD10, KIAA0882, and CMYB. Initial analyses with clinical parameters showed that FOXA1 was higher expressed in G1 and G2 differentiated tumors, in tumors with low Ki67 expression, and in ER- and PR-positive tumors $(\mathrm{p}<0.001)$. For PSMD10, Cox Regression and Kaplan-Meier survival analyses showed that patients having tumors with moderate and high PSMD10 expression had a significantly shorter median overall survival period (5.4 years vs. 10.3 years, $\mathrm{p}=0.013$ ) and a higher Hazard Ratio (HR $=1.71,95 \%$ CI 1.1-2.6, $\mathrm{p}=0.014$ ) compared to patients with no or low PSMD10 expression.

Conclusion: In conclusion, eight out of twelve targets could be evaluated in 405 FFPE tissue samples. FOXA1 showed a significant adverse association with differentiation as well as proliferation grade and hormone receptor status, whereas PSMD10 expression correlated with a worse prognosis. Further statistical and functional validation analyses could confirm the clinical and prognostic relevance of the 12-gene signature.

Background: In locally advanced colon cancer (stage III UICC/AJCC), guidelines recommend adjuvant chemotherapy, in most cases based on the cytotoxic pyrimidine analog 5-FU 
Abstracts: 133 rd Congress of the German Society of Surgery (DGCH)

(5-fluorouracil), which inhibits the thymidylate synthase (TYMS). Even though multimodal treatment prolongs survival in the whole stage III group, a significant fraction of patients will not respond to therapy and suffer from disease relapse. However, with the exception of DNA microsatellite instability, no predictive markers exist to date that allow a reliable identification of the high-risk patient group, which does not benefit from adjuvant therapy. Therefore, this study aimed to validate and expand a biomarker profile, which has previously been developed for stage II colon cancer patients, for its use in personalized treatment decisions, with regards to the individual risk profile (prognosis) and response (prediction).

Materials and Methods: Patients with histologically proven colon cancer stage III (any T-stage, $\mathrm{N}+, \mathrm{M} 0$ ) and complete resection (R0) at the Dept. of Surgery of TUM, or Darmzentrum Vechta, were included in this retrospective analysis. No patient received neoadjuvant treatment. Clinical, histopathological and follow-up data were collected, and tumor as well as non-diseased colon tissue was available for analysis. Molecular genetic analysis included high resolution melting analysis to determine the mutational status of the oncogenes KRAS (exon2), BRAF (exon15), PI3K (exon 9 and 20 ), as well as quantitative real time PCR to determine activation level of canonical WNT pathway (as quantified by the surrogate marker transcript osteopontin), the gene expression of the candidate metastasis biomarkers SASH1 and MACC1, and expression of the 5-FU target TYMS. Additionally, gene expression of the T-lymphocyte marker CD3epsilon was measured to determine the degree of immune infiltration.

Results: Overall, 88 patients with stage III colon cancer were included (58 male and 30 female) with a median age of 67 years. Tumor specific five year survival was $61 \pm 6 \%$ (median follow: 9.5 years). In accordance to previous reports, KRAS mutations occurred in $36 \%$ and BRAF mutations in $10 \%$ of the patients, occurring in a mutually exclusive fashion. Elevated expression of TYMS significantly correlated with upregulation of canonical WNT signaling $(\mathrm{p}=0.024)$, absence of KRAS mutations ( $p=0.029$ ), as well as increased expression of CD3epsilon ( $p=0.029)$, suggesting that molecular interactions play a crucial role for the effect of a $5 \mathrm{FU}$ based chemotherapy. Of all 88 patients, 34 died due to tumor-related caused during the follow up. However, the level of TMYS expression was not associated with survival, suggesting a predictive rather than prognostic role for this potential biomarker. Multivariate analysis showed that grading, tumor stage and KRAS mutation remain as independent prognostic parameters. We are currently evaluating the DNA microsatellite status on genomic DNA, as well as the amount on T-cell infiltration by analysis of tissue sections, to obtain additional information for mutational and immunological stratification.

Conclusion: A previously identified set of biomarkers for stage II colon cancer patients was tested on a bi-centric collective of stage III colon cancer patients. Preliminary evidence suggests that stage-dependent genetic alterations of distinct molecular pathways may facilitate multimodal therapy decisions in the clinical setting.

\section{1}

\section{MiRNAs as Biomarkers in Thyroid Cancer: Correlation with Their Deputed Targets HMGA2 and SLC5A5}

\author{
A. Damanakis, S. Eckhardt, A. Wunderlich, D.K. Bartsch, \\ P. Di Fazio \\ Universitätsklinikum Marburg, Marburg, Germany
}

Background: MiRNAs play a key role in human tumorigenesis. They are characterized by having a tumorsuppressor or oncogenic role based on their ability to block the transcription of their deputed targets. We aimed to examine the expression of miRNAs and their potential correlation with their targets in ATC, PTC and FTC samples collected in our tissue bank.

Materials and Methods: 16 PTC (papillary thyroid carcinoma), 9 FTC (follicular thyroid carcinoma), 13 ATC (anaplastic thyroid carcinoma) and 3 normal thyroid tissue samples were analyzed for the expression of miRNAs hsa-let7b-5p, hsa-let7f-5p, hsa-miR-146b-5p and hsa-miR222-5p and potential targets SLC5A5 and HMGA2 by RT-qPCR. In silico analysis was performed to reveal targets of HMGA2 and SLC5A5.

Results: In silico analysis showed HMGA2 being a validated target of hsa-let7b-5p and hsa-let7f-5p, whereas SLC5A5 was found to be a target of only hsa-let7f-5p.

PTC samples were characterized by up-regulation of all miRNAs. SLC5A5 was strongly suppressed in comparison with normal thyroid tissue. HMGA2 was overexpressed.

FTC patients showed a significant down-regulation of hsa-let7b-5p and its precursor. Hsa-let7f-5p and hsa-miR222$5 p$ were overexpressed, whereas hsa-miR-146b-5p showed a not homogenous expression. SLC5A5 was strongly suppressed, in correlation with its regulator hsa-let7f-5p. HMGA2 was overexpressed in FTC also, reflecting no correlation with its regulatory let7 miRNAs as it does in other malignancies and as it was also expected in TC.

ATC samples showed a similar miRNA profile as PTC. In contrast with FTC and PTC, these patients showed a stable or up-regulated SLC5A5 and HMGA2.

Conclusion: Identification of miRNAs target proteins will help in understanding their role in tumorigenesis better and will further put a focus on influencing miRNAs expression in targeted therapies. 


\section{2}

eNOS Pathway Polymorphisms as Prognostic Markers and Therapeutic Targets In NSCLC

\author{
F.G. Uzunoglu, A. Duhovic, C. Güngör, M. Nentwich, \\ M. Reeh, A. Heumann, K. Pantel, A. Kutup, O. Mann, \\ J.R. Izbicki, H. Wikman, Y. Vashist \\ UK Eppendorf, Hamburg, Germany
}

Background: The progress of non-small cell lung cancer (NSCLC) is dependent on sufficient angiogenesis. Endothelial NO, synthesized from L-arginine by endothelial NO synthase (eNOS), promotes neoangiogenesis and vasculogenesis. It is induced by proangiogenic factors like sphingosine 1-phosphate (S1P), vascular endothelial growth factor (VEGF), platelet-derived growth factor beta polypeptide (PDGFB) and Angiopoietin 1 (Ang1). The aim of this study was to evaluate polymorphisms of the eNOS gene itself or activating factors and their receptors as prognostic markers in NSCLC patients.

Materials and Methods: Genotyping of 228 patients, surgically treated for NSCLC between 1996 and 2012, was carried out by TaqMan genotyping assays or polymerase chain reaction. Chi-square test, Kaplan-Meier estimator and Cox regression hazard model were used to assess the prognostic value of selected polymorphisms. Polymorphisms selected for analyses had to be well documented and established, with probable functional impact, and with a minor homozygous allele frequency of at least 5\% published for Caucasian populations. In total six polymorphisms were analysed (eNOS: $894 \mathrm{G} / \mathrm{T}$ and $27 \mathrm{bp}$ variable number tandem repeat (VNTR) polymorphism in intron 4; S1PR1: $-1577 \mathrm{G} / \mathrm{T}$ and -532 C/G; PDGFB: 1135 A/C; VEGFR2: -2854 G/T and 23408 A/C).

Results: PDGFB $1135 \mathrm{C}$ allele was associated with shorter DFS and OS in univariate analyses and was identified as a negative prognostic marker for DFS and OS in lung adenocarcinoma patients (DFS: harard ratio (HR) 2.08, 95\% confidence interval (CI) 1.21-3.57, p = 0.008; OS: HR 2.24, 95\% CI 1.26$4.00, p=0.006$, figure 1 ). No associations were evident in squamous cell lung carcinoma patients. Remaining polymorphisms showed associations with advanced tumor stage (VEGFR2 $23408 \mathrm{~A} / \mathrm{C}$ ), tumor grading (S1PR1 -1577 G/T) or nodal status (VEGFR2 23408 A/C, eNOS 27 bp VNTR), but no further correlations in survival analyses were evident.

Conclusion: The maturation of VEGF mediated premature vasculatur is dependent on perivascular stromal cells activated by PDGFB. Activation of the PDGFR- $ß$ leads to vasculature and stromal maturation as well as induction of erythropoietin mRNA and protein expression, which promotes tumor angiogenesis. The PDGFB $1135 \mathrm{C}$ allel was shown to be a strong negative prognostic marker in NSCLC patients, indicating a potential biological impact on tumor angiogenesis. Genotyping of the PDGFB $1135 \mathrm{~A}>\mathrm{C}$ polymorphism could be a useful tool to identify high-risk lung adenocarcinoma patients with potential therapeutic impact on targeted therapies.

\section{3}

Serum-Sphingosine-1-Phosphate Concentrations Are Inversely Associated with Atherosclerosis in Humans

\author{
G. Daum, I. Soltau, E. Mudersbach, M. Geissen, \\ E. Schwedhelm, M. Winkler, M. Geffken, S. Peine, \\ S. Debus, A. Larena-Avellaneda \\ UKE, Hamburg, Germany
}

Background: Atherosclerotic changes of arteries are the leading cause for deaths in cardiovascular disease and greatly impair patient\&rsquo;s quality of life. Sphingosine-1-phosphate is a signaling sphingolipid that regulates potentially pro-as well as anti-atherogenic processes. Here, we investigate whether serum-S1P concentrations are associated with peripheral artery disease (PAD) and carotid stenosis (CS).

Materials and Methods: In a prospective study, serum was sampled from blood donors (controls, $\mathrm{n}=174$ ) and from atherosclerotic patients $(n=132)$ who presented to the hospital with either clinically relevant PAD $(n=102)$ or CS $(\mathrm{n}=30)$. From all subjects, serum-S1P was measured by mass spectrometry and blood parameters were determined by routine laboratory assays.

Results: When compared to controls, atherosclerotic patients before invasive treatment to restore blood flow showed significantly lower serum-S1P levels. This difference cannot be explained by risk factors for atherosclerosis (old age, male gender, hypertension, hypercholesteremia, obesity, diabetes or smoking) or comorbidities (COPD, kidney insufficiency or arrhythmia). The best correlation between serumS1P and any blood parameter tested was found for platelets in both controls and patients. Receiver operating characteristic (ROC) curves suggest that S1P has more power to indicate atherosclerosis than HDL. In 35 patients, serum-S1P was measured again between one and six months after treatment. In this group, serum-S1P concentrations rose after treatment independent of whether patients had PAD or CS, or whether they underwent open or endovascular surgery. Post-treatment S1P levels were highly associated to platelet numbers measured pre-treatment.

Conclusion: Our study shows that atherosclerosis in humans is associated with decreased serum-S1P concentrations and that S1P possesses higher accuracy to indicate atherosclerosis than HDL. Based on correlation analyses, platelets appear to play an important role in defining serum$\mathrm{S} 1 \mathrm{P}$ levels in controls and atherosclerotic patients. 


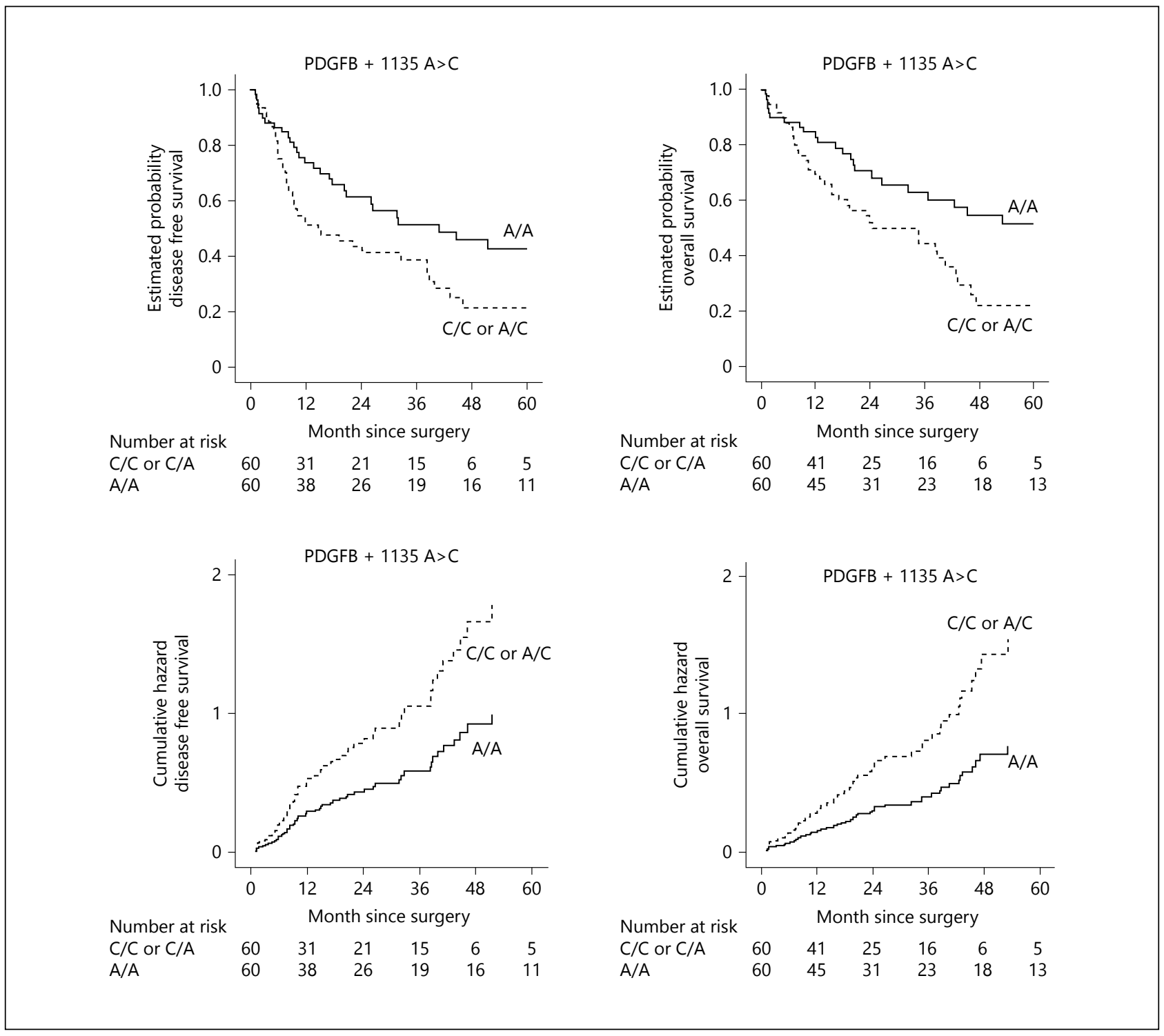

Fig. 1. (for Abstract 32).

\section{4}

\section{Towards Process-Biomarkers in Surgery:} A Semantic, Data-Driven Approach

L.M. Ternes ${ }^{1}$, M. Wagner ${ }^{1}$, M. Apitz ${ }^{1}$, R. Rempel ${ }^{1}$, G. Schneider ${ }^{1}$, T. Weller ${ }^{2}$, M. Maleshkova ${ }^{2}$, B. Bergh ${ }^{1}$, B.P. Müller ${ }^{1}$, H.G. Kenngott ${ }^{1}$

${ }^{1}$ Universitätsklinikum Heidelberg, Heidelberg, ${ }^{2}$ Karlsruher Institut für Technologie, Karlsruhe, Germany

Background: Clinical pathways and standard operating procedures (SOPs) as models for surgical processes have been shown to improve surgical treatment. However, processes in surgery are versatile and often there is not much known about how processes are followed in reality. At the same time, a huge amount of structured data containing information about these real processes already exists in hospital information systems (HIS). Therefore, the aim of our study was to demonstrate the applicability of HIS-data-driven process analysis for surgical treatment processes. Thereby we developed patterns that are predictive to patient outcome and can act as processbiomarkers.

Materials and Methods: Data collection was performed by information retrieval from HIS. Two use cases have been studied. First, data about the perioperative process around the surgery was extracted anonymously for 18.975 cases. Second, data about the pre- and postoperative process from admission to discharge was collected for a group of 30 patients 
Abstracts: 133rd Congress of the German Society of Surgery (DGCH)

within the CoGuS-Study. In discussion with surgical process experts, background knowledge about the connection between data and surgical treatment processes was collected. This knowledge then was formalized in a machine-readable format by semantic description of the data in a comprehensive ontology. The ontology was connected with the data and used to create a knowledge graph for analysis comprising both, surgical knowledge about the process models and data from real processes. Also, the data was analyzed with open-source process mining tools Disco (Fluxicon Process Laboratories, Eindhoven, Netherlands) and ProM (Process Mining group, Eindhoven Technical University, Netherlands).

Results: For the perioperative process 172.331 data points including 11 different activities were extracted. For each data point 5 additional parameters could be extracted. For the preand postoperative process between 1000-6500 data points per case including 410 different activities were extracted. Besides this, 170 additional parameters per case could be extracted. A semantic data model as well as process models and metrics for both processes could be generated. Furthermore, the semantic data model was integrated into the process model to create a semantic, data-driven process model. This model allowed for clustering the patients into groups solely based on process characteristics.

Conclusion: A semantic, data-driven process model was created by utilizing HIS-data. The process mining tools created visualizations of process reality. Furthermore, the generated models offer great potential for further research including comparison to and extension of clinical pathway models, refined patient clustering by means of process steps and the detection and prediction of events such as surgical complications. In the future, process characteristics that are specific to selected patient cohorts can become clinically relevant process-biomarkers.

Acknowledgements: This research was funded by the German Research Foundation (DFG) within the Transregional Collaborative Research Center 125 'Cognition-Guided Surgery' in projects A01 and I01 and by the Medical School of Heidelberg University with a Physician-Scientist-Fellowship for Martin Wagner.

\section{5}

Influence of the Tumor Microenvironment on Proliferation, Vitality and Chemosensitivity in Liposarcomas

M. Becerikli, K. Harati, T. Hirsch, M. Lehnhardt, A. Daigeler, F. Jacobsen

BG Universitätskliniken Bergmannsheil, Bochum, Germany

Background: Soft tissue sarcoma displays a heterogeneous group of mesenchymal tumors. Thereof, liposarcomas represent the second most common type. Liposarcomas can occur at almost all locations of the body and show a poor clinical response to chemotherapy. From various carcinomas it is known, that the tumor microenvironment, particularly the tumor-associated fibroblasts (TAFs) play an essential role in tumor survival and progression. They can release cytokines, proteolytic enzymes, growth factors and remodel the extracellular matrix and hereby influence the tumor progression and invasion. The aim of this study was to investigate the influence of TAFs isolated from liposarcomas on the physiology and metabolism of liposarcoma cells.

Materials and Methods: Intratumoral TAFs were isolated from a myxoid liposarcoma with an intermediate grading (G2) and a more malignant, pleomorphic liposarcoma (G3). Expression of alpha-Smooth Muscle Actin (a-SMA) was determined in the isolated TAFs and in dermal fibroblasts as control by immunofluorescence. The ATCC listed liposarcoma cell line SW872 was co-cultured with the corresponding TAFs or with dermal fibroblasts. Subsequently, cell proliferation (BrdU), vitality (MTT) and chemosensivity (iCELLigence, OLS OMNI Life Science, Bremen) of the co-cultured SW872 cells were examined.

Results: TAFs showed an altered a-SMA expression behavior than dermal fibroblast. Thus, enhancing the medium FCS concentration from $10 \%$ to $20 \%$ increased a-SMA expression in TAFs more than in dermal fibroblasts. The BrdU proliferation assay demonstrated a significant increase in SW872 liposarcoma cell line proliferation after co-cultivation with TAFs compared to co-cultivation with dermal fibroblasts. Interestingly, SW872 co-cultivation with TAFs from grade 3 liposarcoma showed higher proliferation levels than from grade 2 liposarcoma. A similar trend was also found in the MTT analysis. A significant increase in cell vitality was observed in SW872 cells co-cultivated with TAFs than with dermal fibroblasts. Additionally, co-culture with TAFs from grade 3 tumor showed also an increased vitality than TAFs from grade 2 tumor. Finally, over the entire time course of treatment a worse response of SW872 cells to doxorubicin was observed in co-culture with TAFs. Again, the effect was higher in the TAFs isolated from the more malignant grade 3 liposarcoma.

Conclusion: These data provide an evidence for the great influence of the tumor microenvironment on sarcoma cells. TAFs from liposarcomas enhance the proliferation, invasiveness and chemosensivity of SW872 liposarcoma cells significantly compared to normal fibroblasts from the dermis. Furthermore TAFs from more malignant liposarcomas show a larger influence than TAFs from well differentiated less malignant liposarcomas. The study will be expanded to TAFs from more patients of different liposarcoma entities and grades. 
Abstracts: 133 rd Congress of the German Society of Surgery (DGCH)

\section{6 \\ Phenotypic Heterogeneity of Disseminated Tumor Cells Is Predetermined by Primary Tumor Hypoxic Microenvironments}

G. Flügen ${ }^{1}$, A. Avivar-Valderas ${ }^{1}$, Y. Wang ${ }^{2}$, M. Padgen ${ }^{3}$, J. Williams ${ }^{3}$, V. Verkhusha ${ }^{2}$, D. Entenberg ${ }^{2}$, J. Castracane ${ }^{3}$, P. Keely' ${ }^{4}$, J. Condeelis ${ }^{2}$, J. Aguirre-Ghiso ${ }^{1}$

${ }^{1}$ Icahn School of Medicine at Mount Sinai, New York,

${ }^{2}$ Albert Einstein College of Medicine, New York,

${ }^{3}$ SUNY Polytechnic Institute, Albany, ${ }^{4}$ University of

Wisconsin-Madison, Madison, USA

Background: Heterogeneity within and between primary tumors (PTs) is reflected by genetic and epigenetic diversity and varying PT microenvironments. Further, whether PT microenvironments might influence the fate of disseminating tumor cells (DTC) has never been explored. We found that breast tumors enriched for a specific dormancy signature (DS) displayed longer metastasis-free periods than those poor (DS-) for the signature. Key genes in the DS induce quiescence and are also regulated by hypoxia. Clinical evidence links hypoxic tumors to increased therapy resistance and a worse outcome. However, a main response of tumor cells to hypoxia is growth arrest, but how this response is linked to the clinical outcome is unknown. We hypothesized that hypoxic PT microenvironments may spawn a subpopulation of DTCs that, by virtue of becoming dormant, might escape therapies and eventually fuel incurable metastasis.

Results: We used H2B-GFP inducible HEp3 HNSCC and photo-switchable (green-to-red fluorescence) H2B-Dendra2 expressing MDA-MB-231 and ZR-75-1 human breast cancer cell lines to identify cells from hypoxic microenvironments. To initiate spatially defined hypoxic microenvironments in primary tumors we implanted induction NANo IntraVItal Devices (iNANIVIDs) carrying a hypoxia-mimetic agent (desferrioxamine - DFOM) in T-HEp3 tumors in vivo or exposed cultured MDA-MB-231 or ZR-75-1 cells in vitro to either $21 \%$ or $1 \% 02$. The regions influenced by the DFOMiNANIVID displayed significant upregulation of p27, NR2F1 and DEC2 (dormancy genes), as well as induction of hypoxia markers (GLUT1, HIF1a). Human HNSCC PT samples showed the same link between spontaneous hypoxic regions and up regulation of dormancy markers. We further found a significant increase in quiescent lung DTCs of hypoxia induced H2B-GFP T-HEp3 or H2B-Dendra2 MDA-MB-231 cells, traceable $>2$ weeks after extravasation using $\mathrm{H} 2 \mathrm{~B}-\mathrm{GFP}$ and H2B-Dendra2-RED label retention. Using human Vimentin to screen for HEp3 tumor cells in lungs, we found that single, unproliferating DTCs originating from the iNANIVID induced hypoxic regions showed a dormant profile (upregulation of p27, NR2F1, DEC2 and TGFb2) compared to DTCs originating from a normoxic milieu. Simultaneously, only the hypoxic pre-treated group was able to form micro-metastasis at 10 days after injection, suggesting the presence of a more aggressive sub clone in this group. Further, analysis in 3D culture models revealed that ER+/DS+ breast cancer cells (ZR-75-1) are more prone to enter a prolonged quiescent state after a brief exposure to hypoxia $(1 \% 02)$ in an NR2F1dependent manner. This response is not observed in triple negative/DS-breast cancer cells. Lastly, using a spontaneously metastatic PyMT driven Dendra2-tagged breast cancer model, we found that $\sim 75 \%$ of dormant DTCs up regulate the dormancy marker NR2F1 at or soon after reaching the lung, suggesting a rapid induction of dormancy upon reaching target organs.

Conclusion: We propose that hypoxic primary tumor stress microenvironments increase phenotypic heterogeneity of DTCs and lead to the expression of the DS. Upon spreading, these DTCs may be more prone to enter dormancy, evade antiproliferative therapies and eventually fuel metastasis.

\section{7}

\section{HIF-1alpha is Essential for the Pathogenesis of Inflammation-Associated Colon Cancer}

\author{
T. Cramer ${ }^{1}$, M. Erdem ${ }^{1}$, A. Egners ${ }^{1}$, C. Zasada ${ }^{2}$, S. Kempa ${ }^{2}$, \\ N. Rohwer ${ }^{3}$ \\ ${ }^{1}$ Uniklinikum Aachen, Aachen, ${ }^{2} \mathrm{MDC}$, Berlin, ${ }^{3}$ Charité, \\ Berlin, Germany
}

Background: Chronic inflammation is a key factor in the pathogenesis of colon cancer with largely elusive molecular nature. HIF-1a controls central pro-tumorigenic pathways such as glycolysis and angiogenesis. Furthermore, HIF-1a constitutes a pivotal regulator of myeloid cell function and is activated strongly by pro-inflammatory cytokines.

Materials and Methods: We have analyzed inflammationassociated colon cancer growth (AOM+DSS model) in mice lacking HIF-1a specifically in intestinal epithelial or myeloid cells, respectively.

Results: Enterocyte-specific deletion of HIF-1a led to decreased tumor size, while tumor number was not affected. Inflammatory activity was strongly diminished in KO mice. In addition, loss of HIF1A in enterocytes inhibited full activation of the wnt/b-catenin pathway as well as the emergence of tumor-specific metabolic reprogramming. Deletion of HIF-1a in myeloid cells resulted in a significant decrease of both tumor size and tumor number. Remarkably, inflammatory activity in the colon was not affected by the loss of HIF1A in myeloid cells. Adenoma-associated matrix formation and the emergence of tumor-associated fibroblasts were strongly inhibited in myeloid cell-specific HIF1A KO mice. Expression of TGF-b, a central pro-fibrogenic factor, in macrophages was found to be under transcriptional control of HIF1A.

Conclusion: Our results identify HIF1A as a versatile regulator of colitis-associated cancer and point towards a crucial role of enterocytic HIF1A in the control of intestinal inflammation. Furthermore, HIF1A in myeloid cells is pivotal for fibroblast activation and matrix synthesis during intestinal 
adenoma formation. This multilevel importance of HIF1A argues for HIF-1a-inactivating substances as promising agents for the treatment of colon cancer.

\section{8 \\ microRNA-31 Determines Progression-Free Survival after Liver Resection of Colorectal Cancer Metastases \\ C. Franz, S. Stoll, F. Klupp, T. Schmidt, M. Schneider, A. Ulrich \\ Universitätsklinik Heidelberg, Heidelberg, Germany}

Background: microRNAs (miRNAs) are non-coding RNAmolecules with a length of 20-25 nucleotides involved in the posttranscriptional modulation of gene expression. In various studies, miRNAs have proven to play an important role in the carcinogenesis of colorectal cancer as their function can be either tumor suppressive or oncogenic. However, their role in the metastatic progress of colorectal liver metastases is yet to be discovered.

Materials and Methods: 97 patients who underwent liver resection due to colorectal liver metastases at the Department of Surgery, University Clinic of Heidelberg, were included. A panel of five miRNAs (miR-18a, miR-21, miR-31, miR-335, miR-92a) was investigated using fresh frozen tissue samples. Semi-quantitative real-time-PCR was performed with $\delta \delta \mathrm{Ct}$ method and expression of miRNAs was analyzed using the small nuclear RNA (snRNA) RNU6B as endogenous control. Patients were followed up and clinical data was correlated with miRNA expression.

Results: Colorectal liver metastases revealed a significantly higher median expression of miR-18a, miR-21, miR-31, miR-335 and miR-92a (3.6-fold, 6.8-fold, 14.5-fold, 1.2-fold and 2.1-fold, respectively) compared to healthy liver (each $\mathrm{p}<$ 0.001, but miR-335: $p=0.038$ ). Median progression-free survival after liver resection was 12.2 months (95\% CI 10.114.3). In correlation with clinical data higher expression of miR-31 was associated with a significantly reduced progression-free survival of 9.87 months versus 15.03 months in patients with lower expression of miR-31 ( $p=0.011)$.

Conclusion: To our knowledge we are the first to show that higher expression of miR-31 is associated with decreased progression-free survival after resection of colorectal cancer liver metastases. Moreover, miR-18a, miR-21, miR-31, miR-92a and miR-335 were significantly up-regulated in metastatic liver tissue and therefore might play a crucial role in the metastatic progress of colorectal cancer liver metastases.
Fig. 1. Impact of microRNA-31 on progression-free survival after resection of colorectal liver metastases (for Abstract 38).

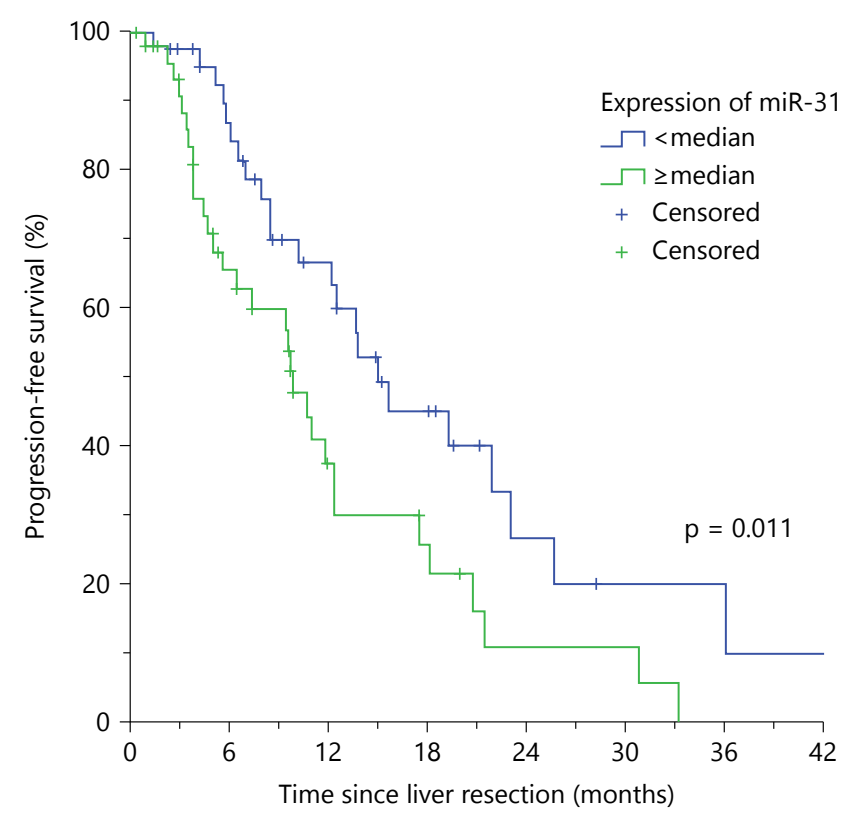




\section{9 \\ Snail and Sonic Hedgehog Activation in Neuroendocrine Typical and Atypical Tumors of the Lung

\author{
C. Meyer, D.K. Bartsch, V. Fendrich, A. Kirschbaum, \\ P. Di Fazio, J. Grups \\ Uniklinik Marburg, Marburg, Germany
}

Background: The transcription factor Snail represses E-cadherin and induces epithelial-mesenchymal transition, a process also exploited by invasive cancer cells. Aberrant Hedgehog (Hh) and snail signaling was recently observed in a variety of neuroendocrine tumors. In this study, we examined for the first time, whether Snail, key regulator of epithelialmesenchymal transition (EMT) and Sonic Hedgehog (SHH) are expressed in neuroendocrine tumors (NETs) of the lung.

Materials and Methods: We used immunohistochemistry and real-time PCR to evaluate the expression of both pathways in resected NETs of the lung. Therfore, the resected tumor tissue of 22 patientsw with NET of the lung, were analyzed. The patient population comprised 10 men and 12 female with a median age of 60 years (range 33-83). We used Snail 1, E-cadherin and Vimentin as markers of EMT and Sonic Hedgehog (Shh), Ptch1 and Smo as marker of the HH signaliing pathway.

Results: Using immunohistochemistry, we found expression of Snail in 15 out of 22 (68\%) of evaluated NET samples, but not in adjacent normal tissues. Snail expression was mostly restricted to the invasive front of the tumors. Intratumoral expression of $\mathrm{SHH}$ was detected in 20 out of 22 (91\%) tumors. E-cadherin was still expressed in all typical NETs of the lung, but absent in the atypical carcinoids. As opposed to Snail, cells expressing SHH were found to be distributed more randomly throughout the tumors. The other markers of the HH pathway were virtually present in all tu or samples. Furthermore, we found downregulation of E-cadherin in Snail-expressing cells by immunofluorescence. Real-time RT-PCR comfirmed the up regulation of the different markers.

Conclusion: In conclusion, we describe for the first time the activation of the EMT and the HH pathway in NETs of the lung. This may play an important role of tumor progression in these type of cancer.

\section{0 \\ Endothelial Cell Activation via von Willebrand Factor in Non Small Cell Lung Carcinoma}

I. Karampinis, J. Koett, A.T. Bauer, S.W. Schneider,

P. Hohenberger, K. Nowak

Universitätsmedizin Mannheim, Mannheim, Germany

Background: Tumor associated coagulopathy is one of the main reasons for the bad prognosis of tumor-patients. The interaction of the tumor cell with the vascular endothelium leads to endothelial cell activation and subsequently to the release of the procoagulatory protein von Willebrand factor (vWF).

This multimere Protein binds efficiently to Thrombocytes and could therefore be causative related with the tumor associated hypercoagulopathy. However, the role of this pathway in the progression and metastasation of lung carcinoma is still unclear.

Materials and Methods: In this study we analyzed 53 Patients with lung carcinoma and compared them with 30 healthy controls from our laboratory staff. The role of the tumor associated endothelial cell activation was examined locally in tissue sections with high-resolution fluorescence microscopy. Additionally we compared the local process with the systemic endothelial activation through measurement of the endothelial markers vWF, Angiopoetin-2 and vascular endothelial growth factor (VEGF) in peripheral blood samples. Furthermore we tested the activity of the vWF degrading enzyme ADAMTS-13. The study was approved by the local ethic committee and all patients participated voluntarily and gave their written consent.

Results: In comparison to sections of healthy tissue we identified intraluminal vWF fibers in micorvascular structures of histological sections of tumour tissue. These vWF fibers have the ability to efficiently bind to Thrombocytes. In the analysis of the peripheral blood of lung cancer patients we manifested significantly higher values of vWF, VEGF, and Angiopoetin-2 than in the healthy controls. The systemic activity of the ADAMTS-13 enzyme was significantly reduced in all tumour patients compared to the healthy controls.

Conclusion: The results of this study support the hypothesis that VEGF-a secretion, induced from the tumour cells, stimulates the release of $\mathrm{vWF}$ and through this pathway the microthrombosis of blood vessels in patients with lung carcinoma. The systemic attenuation of the ADAMTS-13 enzyme activity could also sustain the thrombotic microenvironment through the enhanced prevalence of vWF fibers. However further studies are needed to investigate the exact pathway which leads from tumour induced microthrombosis to the metastasation process. 


\section{1}

Balance of miR-148a Expression Impacts on Chemotherapy Resistance, Tumor Cell Survival and Metastatic Potential of Esophageal Squamous Cell Carcinoma

C. Matuszcak ${ }^{1}$, K. Lindner ${ }^{1}$, A.K. Müller ${ }^{1}$, J. Haier ${ }^{2}$, R. Hummel ${ }^{1}$

${ }^{1}$ Klinik für Allgemein- und Viszeralchirurgie, Münster,

${ }^{2}$ Universitätsklinikum Münster, Münster, Germany

Background: Previously, we found upregulation of miR-148a to enhance sensitivity towards cisplatin and 5-FU in sensitive and resistant esophageal cancer cell lines. The current study now aimed to thoroughly assess the impact of miR-148a on chemotherapy resistance, tumor cell survival and metastatic potential in esophageal squamous cell carcinoma (ESCC).

Materials and Methods: miR-148a expression was manipulated in commercially available ESCC cell lines via transient liposomal transfection. The effect of up- and downregulation on chemotherapy resistance was assessed in six cell lines. Impact of miR-148a expression on apoptosis, cell cycle, adhesion and migration was investigated in two cell lines using standard assays. Expression of potential targets was then analyzed via western blot.

Results: Upregulation of miR-148a significantly increased sensitivity towards cisplatin in 5 out of 6 cell lines $(-13 \%$ to $-30 \%, \mathrm{p}=\leq 0.043$ ), and towards 5 -FU in 2 out of 6 cell lines $(-7 \%$ to $-11 \%, \mathrm{p}=\leq 0.002)$ as expected from previous experiments. Surprisingly, downregulation of miR-148a also improved sensitivity towards cisplatin by $-7 \%$ to $-30 \%$ ( $\mathrm{p}=$ $\leq 0.035)$ and towards 5 -FU by $-8 \%$ to $-31 \%(p=\leq 0.012)$ in 5 respectively 4 out of 6 cell lines. Furthermore, up- as well as downregulation of miR-148a led to a significant increase in apoptosis (33-120\%, p = $\leq 0.048)$ ), but did not affect cell cycle regulation. In addition, ability of cells to adhere ( -26 to $-30 \%$, $\mathrm{p}=\leq 0.040)$ and migrate $(-22$ to $-49 \%, \mathrm{p}=\leq 0.005)$ was significantly reduced after up- or downregulation. Western blot analysis showed that pro-apoptotic Bim and anti-apoptotic Bcl-2 were deregulated after miR-148a upregulation on protein level as expected from negative posttranscriptional control via miR-148a. Statistical significance was assessed using parametric tests (Student's t-test for equal and ManWhitney-U-test for unequal variances).

Conclusion: Our study showed for the first time that both, up- and downregulation of miR-148a, impacted similarly on chemotherapy resistance, tumor cell survival and metastatic potential in ESCC cell lines, and we found pro-apoptotic protein Bim and anti-apoptotic protein Bcl-2 as potential targets. These data suggest that miR-148a regulates apoptosis on various levels in different directions, and balance of miR-148a expression might play a crucial role in chemotherapy resistance and cancer behavior. Further studies are needed to fully understand miR-148a effect on the downstream pathways, and to investigate its clinical potential.

\section{2}

Limitations of Liquid Biopsies for Genomic

Analyses in Locally Advanced Esophageal Cancer Patients at the Time of Primary Staging

S. Chon ${ }^{1}$, S.P. Mönig ${ }^{1}$, A. Hölscher ${ }^{1}$, E. Bollschweiler ${ }^{1}$, P.S. Plum ${ }^{1}$, H. Schlösser ${ }^{1}$, U. Warnecke-Eberz ${ }^{1}$, H. Pasternack ${ }^{1}$, B. Polzer ${ }^{2}$, R. Büttner ${ }^{1}$, J. Weiss ${ }^{1}$, T. Herbold ${ }^{1}$, T. Zander ${ }^{1}$, A. Quass ${ }^{1}$, H. Alakus ${ }^{1}$

${ }^{1}$ Universität zu Köln, Köln, ${ }^{2}$ Fraunhofer ITEM-R, Regensburg, Germany

Background: Esophageal cancer (EC) is among the ten most common malignancies worldwide. Over the last two decades, the incidence of esophageal adenocarcinoma (EAC) has risen more rapidly than any other malignancy in North America and Western Europe. In recent years, high-throughput technologies have uncovered driver events and the mutational complexity of EC in a comprehensive scale. Genomic profiling of primary tumor foci, corresponding circulating tumor cells (CTC) and analysis of circulating cell-free DNA (cfDNA) in patients with EC during the course of treatment (staging, neoadjuvant treatment, restaging and surgery) is thought to play an increasing role in future to improve the efficacy of personalized treatment. The present study was therefore aimed to study the feasibility of using liquid biopsies for genomic profiling in EC patients.

Materials and Methods: The first part of this study was designed as a proof-of-principle experiment by including 8 EC patients with a locally advanced tumor stage followed by adding 12 further patients. A total of $30 \mathrm{ml}$ blood [20 $\mathrm{ml}$ EDTA, $10 \mathrm{ml}$ in CellSave $\left.{ }^{\circledR}\right]$ and frozen tumor biopsies were collected during routine endoscopy at the time of primary staging. EDTA blood samples were centrifuged to separate plasma. cfDNA was then extracted from plasma with the use of the QIAamp circulating nucleic acid kit (Qiagen). DNA concentrations were determined by fluorometry $\left(Q_{\text {ubit }}{ }^{\circledR}\right.$, Life Technologies) and by qPCR. Blood collected in CellSave ${ }^{\circledR}$ tubes were processed for the enumeration of circulating tumor cells (CTCs) using the CellSearch ${ }^{\circledR}$ system (Veridex). Tumor biopsies were evaluated for tumor cell content by cryosectioning and HaE-staining followed by DNA extraction (QIAmp DNA Micro kit). DNA from biopsies and plasma were then used for library construction for Next-Generation-Sequencing (Ion AmpliSeq\&trade; Life Technologies) and ultra-deepsequencing (UDT-sequencing) was performed on a 12 gene panel (TP53, PIK3CA, KRAS, NRAS etc.) with a coverage of $>1000 x$ on a MiSeq (Illumina).

Results: The majority of patients (65\%) showed detectable mutations in their tumor biopsies. In contrast to tumor biopsies, mutations in cfDNA were detectable only in the minority of patients (12\%). The detected mutations in cfDNA were the same as in the primary tumor biopsies but with a much smaller allele frequency $(<2 \%)$. CTCs were also only detectable in the minority of patients (15\%). Experiments 
Abstracts: 133 rd Congress of the German Society of Surgery (DGCH)

including metastatic patients with different tumor types showed much higher detection rates for mutated cfDNA and for CTCs revealing the systematic tumor burden and the low amount of cfDNA with $<500$ genomic copies per $2 \mathrm{ml}$ plasma as two main factors for low detection rates of CTCs in our patients.

Conclusion: Liquid biopsies for genomic profiling of CTCs and cfDNA in locally advanced EC patients is technically possible and can further be optimized (specific EC gene panel, high-coverage etc.). But the major limiting factors resulting in low detection rates of mutations in these biopsies are more biological factors (low systematic tumor burden and low genomic copies per plasma) that cannot easily be overcome in these patients.

\section{3 \\ Comparison of the Prognostic Relevance of Circulating Tumor Cells in Blood and Disseminated Tumor Cells in Bone Marrow of Patients with Esophageal Cancer}

M. Reeh, S. Riethdorf, F.G. Uzunoglu, T. Ghadban, M. Nentwich, Y. Vashist, K. Effenberger, K. Pantel, J.R. Izbicki, M. Bockhorn

Universitätsklinikum Hamburg-Eppendorf, Hamburg, Germany

Background: Current staging methods for esophageal cancer (EC) need to be improved for sufficient treatment stratification and individual risk prediction. Because hematogenous tumor cell dissemination is a key event in tumor progression, we assessed the prognostic significance of disseminated tumor cells (DTC) in bone marrow and circulating tumor cells (CTC) in peripheral blood from patients with EC.

Materials and Methods: In this prospective, singlecenter study, from 120 patients with preoperatively nonmetastatic staged EC, 76 patients had usable bone marrow aspirates as well as peripheral blood probes. The CellSearch system was used to enumerate CTCs. Bone marrow was aspirated from the iliac crest were enriched by Ficoll density gradient centrifugation. To detect DTCs were immunostained with the pan-keratin antibody A45-B/B3. Results were correlated with clinicopathologic parameters and clinical outcome such as recurrence and death during followup time.

Results: Fifteen of 76 patients (19.7\%) harbored CTCs in peripheral blood, whereas in 13 of 76 patients (17.1\%) DTCs in bone marrow could be detected. In only 3 patients (3.9\%) CTCs and DTCs were detected simultaneously, whereas concordant results (DTC/CTC neg. and DTC/CTC pos.) were found in 54 patients (71.1\%). Surprisingly, only patients with CTCs showed significant shorter overall survival and relapsefree survival in contrast to patients with DTCs ( $p=0.019$ and $\mathrm{p}=0.002$, respectively). Multivariate analyses revealed that only CTC status was a significant and independent predictor of overall survival and relapse-free survival $(\mathrm{p}=0.013$ and $\mathrm{p}<$ 0.001 , respectively).

Conclusion: This is the first study analyzing CTC and DTC status in one cohort of patients with EC. Only the CTC status seems to be an independent, prognostic marker suitable and easy-to-use for clinical staging of patients with EC.
44
Consequences of Complete Remission (ypT0) after Neoadjuvant Chemotherapy in Gastric and Esophageal Adenocarcinoma
H. Nienhüser ${ }^{1}$, S.B. Blank ${ }^{1}$, L. Sisic ${ }^{1}$, U. Heger ${ }^{1}$, W. Weichert ${ }^{2}$, D. Jäger ${ }^{3}$, A. Ulrich ${ }^{1}$, M.W. Büchler ${ }^{1}$, K. Ott ${ }^{4}, T$. Schmidt
${ }^{1}$ Universitätsklinikum Heidelberg, Heidelberg,
${ }^{2}$ Technische Universität München, München, ${ }^{3}$ Universität Heidelberg, Heidelberg, ${ }^{4}$ Klinikum Rosenheim,
Rosenheim, Germany

Background: Neoadjuvant therapy of gastric and esophageal cancer led to increase in overall survival in the last decade. In cases of excellent response to chemotherapy the tumor is histologically not detectable (ypT0). In this case the necessity of an operation and the consequences for the patient has to be discussed.

Materials and Methods: From 2001 to 2015440 neoadjuvantly treated patients with gastric and esophageal adenocarcinoma were operated at the University Hospital of Heidelberg. In 35 of these patients the histological examination showed complete regression of the tumor (ypT0). The clinical data of these patients was analysed with focus on overall survival and frequency of recurrence.

Results: Most of the patients $(80.0 \%, 28 / 35)$ were operated due to adenocarcinom of the esophageal gastric junction (AEG I: $n=17$, AEG II: $n=9$, AEG III: $n=2) .20 .0 \%$ (8/35) of the patients were diagnosed with gastric cancer. The surgical procedure was an abdomino-thoracic esophageal resection in $60.0 \%$ of the cases, in $17.1 \%$ a transhiatal gastrectomy respectively a subtotal gastrectomy was performed, in $5.7 \%$ the procedure was a total gastrectomy. In the histological examination all included patients showed ypT0-category, the median of the resected lymph nodes was $21.5( \pm 11.2)$. In $8.6 \%$ $(\mathrm{n}=3)$ histological examination showed lymph node metastasis, in $5.7 \%$ patients were diagnosed with a pN1-category, one patient $(2.9 \%)$ had a pN2-category. In all of these patients there was suspicion of positive lymph nodes in the preoperative staging $(\mathrm{cN}+)$.

Out the 35 included patients $17.1 \%(n=6)$ had tumor recurrence, median recurrence free survival was $6.3( \pm 2.2)$ months. In the group of patients with positive lymph nodes one patient had tumor recurrence and showed liver metas- 
Abstracts: 133 rd Congress of the German Society of Surgery (DGCH)

tasis. All patients with tumor recurrence $(n=6)$ had distant metastasis (liver $n=2$, lung $n=3$, Krukenberg tumor $n=1$, bone $n=1$ ), one patient had a tumor suspicious liver lesion in the preoperative staging. Local recurrence was diagnosed in only one patient $(2.7 \%)$. Median overall survival in all patients with ypT0-category was $67.9( \pm 7.0)$, in case of ypN0-category median overall survival was $71.0( \pm 7.3)$ months. In patients with positive lymph nodes median overall survival was reduced compared to the group of patients with ypN0category (38.2 \pm 11.5 months, $p=0.24)$. The neoadjuvant therapy was according to Epirubicin-based protocols (ECF, ECX or EOX) in $47.2 \%(n=17)$ of the cases, in $27.8 \%(n=10)$ patients were treated according to the FLOT-protocol, $25 \%$ $(n=9)$ of the patients received other chemotherapies.

Conclusion: Complete tumor remission after neoadjuvant chemotherapy is rare and incidence should be increased by treatment with effective chemotherapy protocols because this group of patient has a significant longer overall survival. There is a relevant number of patient with complete tumor remission has lymph node metastasis which indicates surgical resection of the primary tumor. Additionally there is a relevant risk of distant metastasis which indicates completion of the perioperative therapy even in case of complete remission of the primary tumor.

\section{5}

\section{Immune Checkpoints PDL-1 and CTLA-4 in Gastric Adenocarcinoma}

H.A. Schlößer, U. Drebber, M. Kloth, M. Thelen,

F. Berlth, M. Garcia-Marquez, K. Wennhold,

A. Urbanski, H. Alakus, A. Shimabukuro-Vornhagen,

U. Warnecke-Eberz, A. Hölscher, T. Zander ${ }^{1}$, S.P. Mönig,

M. von Bergwelt-Baildon

Uniklinik Köln, Köln, Germany

Background: Recently, remarkable efficacy of immune checkpoint inhibition has been reported for several types of solid tumors. This study is a comprehensive analysis of cytotoxic $\mathrm{T}$ lymphocyte antigen 4 (CTLA-4) and programmed death 1 ligand 1 (PD-L1) expression in gastric adenocarcinoma and the first study integrating immune checkpoint expression and somatic mutational analysis.

Materials and Methods: PD-L1 and CTLA- 4 were stained on tumor sections of 127 Caucasian patients with gastric adenocarcinoma by immunohistochemistry. Somatic mutation profiling was performed using targeted next-generation sequencing. Expression of PD-L1 and CTLA-4 on lymphocytes in tumor sections, tumor-draining lymph nodes and peripheral blood were studied by flow cytometry and immune-fluorescence microscopy in an additional cohort.

Results: PD-L1 and CTLA-4 were expressed in 44.9\% (57/127) and $86.6 \%(110 / 127)$ of the analyzed gastric adenocarcinoma samples, respectively. CTLA-4 negativity was correlated with higher tumor grading and diffuse subtype according to the Lauren classification. Positive tumor cell staining for PD-L1 or CTLA-4 was associated with inferior overall survival. Expression of PD-1 (52.2\%), PD-L1 (42.2\%) and CTLA-4 $(1.6 \%)$ on tumor infiltrating T cells was significantly elevated compared to peripheral blood lymphocytes. Somatic mutational analysis did not reveal a correlation to expression of PD-L1 or CTLA-4 on tumor cells.

Conclusion: Our analysis revealed a strong association of PD-1/PD-L1 and CTLA-4 with biology of gastric adenocarcinoma. Hence, specific immune checkpoint-inhibitors should be evaluated in this disease. Additionally, the combination of molecular targeted therapies and checkpoint inhibition might be of benefit. An extensive immune monitoring should accompany these studies.

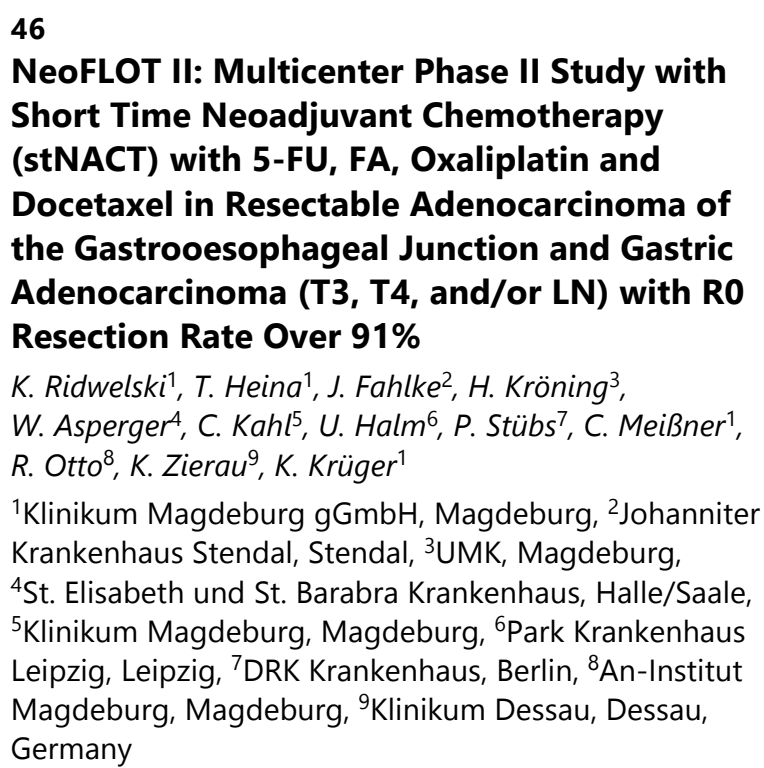

${ }^{1}$ Klinikum Magdeburg gGmbH, Magdeburg, ${ }^{2}$ Johanniter Krankenhaus Stendal, Stendal, ${ }^{3}$ UMK, Magdeburg,

${ }^{4}$ St. Elisabeth und St. Barabra Krankenhaus, Halle/Saale,

${ }^{5}$ Klinikum Magdeburg, Magdeburg, ${ }^{6}$ Park Krankenhaus

Leipzig, Leipzig, ${ }^{7}$ DRK Krankenhaus, Berlin, ${ }^{8} \mathrm{An}$-Institut Magdeburg, Magdeburg, ${ }^{9}$ Klinikum Dessau, Dessau, Germany

Background: Neoadjuvant chemotherapy has gained great importance in the therapy of gastric cancer in recent years, as clinical studies have proven efficacy by showing extended overall survival. Particularly good results were demonstrated in locally advanced, initially non-resectable tumors without distant metastases. Neoadjuvant therapy achieved high remission rates often providing secondary resectability. These results were confirmed in numerous mostly small phase II studies comprising 20 to 80 patients. Al-Batran et al, a study group of the Society of Medical Oncology, have evaluated efficacy, safety and tolerability of Docetaxel combined with 5-fluoruracil, Leucovorin and Oxaliplatin (FLOT) in palliative patients. Apart from determining the effectiveness, the study aimed to optimize the profile of side effects of Docetaxel combining it with a Platin derivate, 5-FU and Leucovorin. Decreasing the rate of hematological side effects was a main objective. Treatment was tolerated well. The most common non-hematological NCI-CTC-grade 3 
Abstracts: 133 rd Congress of the German Society of Surgery (DGCH)

or 4 toxicities were diarrhea (14.8\%), fatigue $(11.1 \%)$ and peripheral neuropathy (9.3\%). NCI-CTC-grade 3 or 4 hematological toxicities neutropenia, leucopenia and thrombopenia were observed in $48.1 \%, 27.8 \%$ and $1.9 \%$ respectively. Febrile neutropenia was observed in 2 patients. No toxicity-related fatalities were recorded.

\section{Materials and Methods:}

This is an open, non randomized Phase II study. For stNACT,

5-FU $\quad 2600 \mathrm{mg} / \mathrm{sqm} / 24 \mathrm{~h}$ on day (D) 1 ,

FA $\quad 200 \mathrm{mg} / \mathrm{sqm} / 30 \mathrm{~min}$ on D 1,

Oxaliplatin $85 \mathrm{mg} / \mathrm{sqm} / 1 \mathrm{~h}$ on D 1 and

Docetaxel $50 \mathrm{mg} / \mathrm{sqm} / 2 \mathrm{~h}$ on D 1

admistrated every 2 weeks for 4 cycles.

Application of adjuvant chemotherapy was not part of the protocol.

\section{Results:}

46 pts. completed 4 cycles stNACT.

1 patient only received 1 cycle and

2 pts. were given 3 cycles only.

47 patients underwent surgery.

1 patient died before surgery.

R0-resection rate was $91.5 \%$ (43/47).

Remission rate

$\begin{array}{lrr}\text { pCR } & 0 & 0 \\ \text { pPR } & 19 & 45.2 \% \\ \text { PD } & 3 & 6.1 \%\end{array}$

Rate of surgical complications

$\begin{array}{lrr}\text { Morbidity } & 12 & 25.5 \% \\ \text { Lethality } & 2 & 4.2 \%\end{array}$

Conclusion: StNACT with only 4 cycles of FLOT is effective and tolerable in resectable GEC.

R0-resection rate was very high.

Overall, the FLOT regimen has proven to be highly effective as neoadjuvant treatment in advanced gastric cancer with tolerable and manageable toxicity.

\section{7}

\section{Suppression of Pancreatic Cancer Progression} by Parasympathetic Signaling

B.W. Renz ${ }^{1}$, M. Macchini ${ }^{2}$, Y. Hayakawa ${ }^{2}$, M. Ilmer ${ }^{1}$, X. Chen ${ }^{2}$, C.B. Westphalen ${ }^{1}$, D.L. Worthley ${ }^{2}$, A.C. luga ${ }^{2}$, J. Werner ${ }^{1}$, A. Kleespies ${ }^{1}$, K.P. Olive ${ }^{2}$, T.C. Wang ${ }^{2}$

${ }^{1}$ LMU Klinikum der Universität München, München, Germany; ${ }^{2}$ Columbia University Medical Center, New York, USA

Background: The parasympathetic nervous system plays an important role in the regulation of epithelial homeostasis and has also been postulated to play a role in tumorigenesis. However, the exact role of the vagus nerve in pancreatic carci- nogenesis is not well understood. Here we study the effects of muscarinic signaling on pancreatic tumorigenesis in a genetically engineered mouse model of pancreatic cancer (PDx1-Cre/ KRasG12D (KC)).

Materials and Methods: KC mice were vagotomized and put on regular water or water supplemented with a muscarinic agonist. Pancreatic tissue was collected and analyzed by immunohistochemistry and RT-PCR at 20 weeks of age; pancreatic cells were isolated and assayed forsphere forming assays. Different human (AsPC-1, BxPC-3, Mia PaCa-2, Panc-1) and murine (K-2548 and K-8282) pancreatic cancer cell lines were subjected to cholinergic and anti-cholinergic drugs and assayed by RT-PCR, Western blot and flow cytometry. Both copies of the muscarinic receptor M3 were deleted in the pancreas in KC mice by breeding them to Lox-M3R-Lox mice.

Results: In pancreatic organoid cultures derived from pancreata harboring an oncogenic KRas mutation, cholinergic agonists suppressed sphere formation significantly. Furthermore, pharmacological inhibition or genetic knockout of the muscarinic M3 receptor abolished this effect in vitro. In human and murine pancreatic cancer cells, anchorage independent growth and tumor sphere forming capacity were reduced by pretreatment with cholinergic agonists. Further evaluation revealed that parasympathetic agonists decrease the CD44 CD24 EpCAM cancer stem cell population in part via inhibition of the mTOR pathway. When Panc-1 cells pretreated with direct parasympathetic agonists were assayed in a xenograft model, they formed less and smaller tumors than the PBS treated cells. Vagotomy, when performed in KC mice at 8 weeks resulted in pancreatic cancer development in $20 \%$ of the animals at 20 week of age. The regular KC phenotype was rescued by administration of a direct muscarinic agonist (Bethanechol) in vagotomized mice. This was reproduced in mice in which the M3 receptor knock out was restricted to the pancreas (PDx1-Cre;KRasG12D;M3RF/F (KC-M3R)). Additionally, treatment of (PDx1-Cre/KRasG12D/p53R172H (KPC)) mice, which had already developed pancreatic cancer, with Bethanechol and gemcitabine prolonged the overall survival significantly in comparison to the gemcitabine treated group.

Conclusion: Taken together, our findings suggest that vagal innervation has a regulatory role in pancreatic tumorigenesis via M3 receptor-mediated suppression of cancer stem cells. As each oncological resection of the pancreatic head is compulsorily associated with a parasympathetic denervation of the pancreas, and thereby a loss of its suppressive effect, this fact might partly explain the high local recurrence rate of this dismal disease. In order to overcome this problem an additional treatment with cholinergic drugs might be necessary. 


\section{8 \\ Targeting TRAIL-R4 as a Novel Option in Pancreatic Cancer Therapy}

J. Gundlach 1 , D. Tawfik², A. Annewanter ${ }^{2}$, K. Legler²,

C. Hauser ${ }^{1}$, J.H. Egberts ${ }^{1}$, S. Sebens ${ }^{2}$, T. Becker ${ }^{1}$,

H. Kalthoff ${ }^{3}$, A. Trauzold ${ }^{2}$

${ }^{1}$ University Hospital Schleswig-Holstein, Campus Kiel, Kiel, ${ }^{2}$ University of Kiel, Institute for Experimental

Cancer Research, CCC-North, Kiel, ${ }^{3}$ Universitätsklinikum

Schleswig-Holstein, Campus Kiel, Kiel, Germany

Background: Pancreatic ductal adenocarcinoma (PDAC) is still a fatal disease and thus novel therapeutic strategies are urgently needed. The death ligand TRAIL is regarded as promising anti-tumor agent due to its ability to induce death preferentially in tumor cells. However, we found that in PDAC cells it also activates pro-inflammatory signaling pathways promoting invasion and metastasis, which clearly limits its curative potential. All known TRAIL-induced effects are attributed to the function of its receptors TRAIL-R1 and/ or-R2 at the cell surface. Tumor cells frequently overexpress these receptors but interestingly, mainly in intracellular locations. Recently we found that intracellular TRAIL-R2 contributes to PDAC malignancy and its high nuclear expression correlates with worse patients\&rsquo; prognosis. Thus, we proposed TRAIL-R2-inhibition as a promising therapeutic option. In addition to TRAIL-R2, TRAIL-R4 is frequently overexpressed in tumors. As a plasma membrane-located receptor without a functional death domain, TRAIL-R4 inhibits TRAIL-mediated cell death via interaction with TRAIL-R1/-R2 and by competing for TRAIL. Similarly to TRAIL-R2, TRAIL-R4 is expressed mainly intracellularly in tumor cells. Its intracellular, ligand-independent functions are completely unknown so far. In the present study, we aimed to understand the role of TRAIL-R4 in PDAC.

Materials and Methods: Protein expression was assessed by Western blots, immunofluorescence and immunohistochemistry. Cell death was studied by crystal violet, PI-staining and FACS analyses. Cell migration and proliferation were evaluated by scratch assay and cell counting, respectively. To determine the impact of TRAIL-R4 on tumor growth, TRAILR4-overexpressing- and control cells were subcutaneously and orthotopically injected into SCID-beige mice. Gene expression analyses were performed using Agilent Arrays and Real-time PCR.

Results: To study the impact of TRAIL-R4 on PDAC cell biology in vitro we generated PDAC cell lines stably overexpressing TRAIL-R4. As expected, overexpression of TRAIL-R4 inhibited TRAIL-mediated apoptosis. In addition, it also resulted in an enhanced cell migration. Furthermore, cell counting demonstrated a significantly increased proliferation of TRAIL-R4-overexpressing cells compared to control cells. In agreement, we detected an enhanced phosphorylation of ERK1/ERK2 in these cells. These effects were still observed in cells treated with neutralizing anti-TRAIL-anti- bodies and thus can be regarded as TRAIL-independent. Through analyzing the gene expression data, we identified genes implicated in inflammation (i.e. IL-6, IL-8) and cell adhesion (i.e. CEACAM5 and CEACAM6) as being most effectively dysregulated. Again, these effects could not be inhibited by neutralization of endogenous TRAIL. Importantly, overexpression of TRAIL-R4 increased tumor growth in subcutaneous and orthotopic xenotransplantation PDAC model in mouse. Finally, immunohistochemical analyses of tissue samples from PDAC patients $(n=106)$ revealed a strong enhancement of intracellular TRAIL-R4 levels compared to the peritumoral adjacent non-malignant pancreatic tissue with high TRAIL-R4-levels representing a negative prognostic marker ( $\mathrm{p}=0.027)$.

Conclusion: Summing up, our in vitro and in vivo/in situ data demonstrated TRAIL-R4 as a significant contributor to PDAC malignancy. Therefore, TRAIL-R4 inhibition might be a beneficial new option in targeted therapy of pancreatic cancer.

\section{9 \\ Genetic Abrogation of Snail Inhibits Acinar-to-Ductal Formation in Precursor Lesions of Pancreatic Ductal Adenocarcinoma

\author{
F. Jendryschek ${ }^{1}$, M. Albers ${ }^{1}$, M. Lauth ${ }^{2}$, A. Baier ${ }^{1}$, \\ D.K. Bartsch ${ }^{1}$, J. Waldmann ${ }^{1}$, V. Fendrich ${ }^{1}$ \\ ${ }^{1}$ UKGM Marburg, Marburg, ${ }^{2}$ Philipps-Universität \\ Marburg, Marburg, Germany
}

Background: Adenocarcinoma of the pancreas is the fourth leading cause of cancer related deaths in Germany. The five-year survival is still less than $8 \%$ and the number of annual incidence and mortality are almost identical. Epthelial-Mesenchymal Transistion is known to be a critical step in tumorigenesis of epithelial carcinomas. Studies have shown that the EMT regulatory protein Snail is overexpressed in pancreatic cancer cells which correlates with invasiveness and metastatic ability. It has also been demonstrated that EMT preceeds pancreatic tumor formation an oocurs in precursor lesions of pancreatic ductal adenocarcinoma. Aim of this study was to compare homozygous and heterozygous Snaildel1;Pdx-1-Cre;LSL-KrasG12D/+_mice with Pdx-1-Cre;LSL-KrasG12D/+-mice in order to draw conclusions about the influence of Snail in initiation and progression of PanINs.

Materials and Methods: Mouse strains of Pdx-1-Cre;LSLKrasG12D/+ and Snail-/- were crossed to generate Snaildel1; Pdx-1-Cre;LSL-KrasG12D/+-mice. Groups of those mice were observed and sacrificed at a defined age. The pancreas was harvested for further histopathological analyses, immunostaining and real-time PCR.

Results: Quantification of PanINs showed a significantly delayed initiation and progression of PanIn lesions at all ages in both homozygous and heterozygous Snaildel1;Pdx- 
Abstracts: 133rd Congress of the German Society of Surgery (DGCH)

1-Cre;LSL-KrasG12D/+-mice. At 4 months of age pancreata of Snaildel1;Pdx-1-Cre;LSL-KrasG12D/+-mice showed up to $100 \%$ normal ducts. Whereas control mice only showed $40 \%$ normal ducts $(\mathrm{p}<0.001)$. At 10 months of age Snaildel1;Pdx1-Cre;LSL-KrasG12D/+-mice had 65\% normal ducts versus $10 \%$ in control mice $(\mathrm{p}<0.02)$. Less than $10 \%$ were higher grade PanINs such as PanIN-2. PanIN-3 lesions were not observed in Snail-Knock-Out mice. The majority of lesions observed in control mice were PanIN-1a and PanIN-1b (31 and 47\%). 2\% were PanIn-3 lesions. Invasive carcinoma was not observed in any of the groups. Using immunostaining and real-time-PCR we found a significant downregulation of Snail and Sox-9 ( $\mathrm{p}<0.05)$.

Conclusion: This study provides in vivo evidence that progression of pancreatic intraepithelial neoplasias dramatically delayed under genetic abrogation of Snail. Further studies will be needed to examine the clinical relevance of those results. Especially Snail as a biomarker or therapeutic target should be evaluated.

\section{0 \\ Xenograft Engraftment and Stromal Activation of Pancreatic Adenocarcinoma Are Predictors for Poor Survival \\ S. Küsters ${ }^{1}$, T. Kees ${ }^{2}$, V. Vuaroqueaux ${ }^{2}$, H.H. Fiebig ${ }^{2}$, \\ U.T. Hopt ${ }^{1}$, U. Wittel ${ }^{1}$, P. Bronsert ${ }^{1}$ \\ ${ }^{1}$ Universitätsklinik Freiburg, Freiburg, ${ }^{2}$ Oncotest, Freiburg, Germany}

Background: Tumor stroma contributes up to $90 \%$ of the pancreatic tumor mass and activation status of the stroma has prognostic implications. Since it is known that in Patientderived xenograft models human stroma is replenished by murine components, it is of interest if stromal characteristics can be transferred from patient tumor (PT) to the xenograft model (PDX) and if stromal characteristics of PDX models can be correlated to clinical parameters.

Materials and Methods: We established of a collection of 42 pancreatic cancer PDX models. Stroma content and stroma activation was evaluated in PT and PDX and correlated to patient survival and tumor grading. Stromal content and activation was assessed using dedicated histological characteristics including fibroblast morphology.

Results: Although stromal contents in PDX models were lower than in PT, high stroma PT were frequently translating into high stromal PDX models $(\mathrm{p}=0.03)$. Morphological signs of stroma activation were similar in PT and PDX. Stromal activation but not stroma content of PT correlated with PDX engraftment rate $(p=0.012)$. Patient survival correlated with stromal activation in PT and corresponding PDX ( $p<$ $0.0001 / p=0.016$ ). We also found a significant longer survival in patients with xenograft engraftment failure compared to patients with a successful engraftment.
Conclusion: We could show that PDX models preserve important elements of stromal biology. Stromal activation and successful xenograft engraftment were predictors for poor survival after resection of pancreatic adenocarcinoma.

51

Preoperative Biliary Stenting versus Early Surgery in Patients with Jaundice Due to Malignant Lesions of the Head of the Pancreas - A Meta-Analysis of Current Literature

F. Scheufele, S. Schorn, C. Jäger, H. Friess, G. Ceyhan

Klinikum Rechts der Isar, TU München, München, Germany

Background: Patients suffering from pancreatic cancer frequently present with obstructive jaundice. While older studies demonstrated a beneficial effect of preoperative biliary decompression, recent studies revealed no significant improvements of PBD on postoperative outcome, but an increment of complications, mostly of infectious nature. However, to date there is no consensus, whether to decompress obstructive jaundice prior to surgery or proceed directly to resection. Thus we aimed to elucidate the impact of PBD of obstructive jaundice due to carcinoma of the head of the pancreas on postoperative morbidity and mortality.

Materials and Methods: For this, we performed a metaanalysis adhering to the PRISMA guidelines, searching medical databases for relevant terms and analyzing the results according to predefined criteria, finally pooling incidence of overall complications, wound infection, pancreas fistula, intra-abdominal abscess and death within the perioperative time period.

Results: 1813 studies were initially identified and finally 22 studies (19 retrospective cohort studies and 3 randomized controlled trials) were included in the analysis with a total number of 4600 patients. Analysis revealed an increased incidence of overall complications (OR of 1.41 [CI.95: 1.07-1.87; $\mathrm{p}=0.02$ ]) and wound infections (OR of 1.89 [CI.95: 1.35-2.63; $\mathrm{p}<0.001]$ ) in patients receiving preoperative biliary drainage compared to direct surgery. Mortality, incidence of pancreatic fistula or intra-abdominal abscess formation were not affected by PBD.

Conclusion: PBD does not have a beneficial effect on postoperative outcome. The clear increase of postoperative overall complications and wound infections, urges for clear indications for PBD and against routinely preoperative biliary decompression. 
Abstracts: 133 rd Congress of the German Society of Surgery (DGCH)

\section{2 \\ The Benefit of Surveillance for Familial Pancreatic Cancer: Outcome of Long-Term Prospective Follow-Up Studies from Three European Expert Centers

\author{
E. Slater ${ }^{1}$, H.F.A. Vasen ${ }^{2}$, I. Ibrahim ${ }^{2}$, J. Earl ${ }^{3}$, E. Matthäi ${ }^{1}$, \\ C. Guillen Ponce ${ }^{3}$, M. Angeles Vaz Salgado 3 , \\ A. Carrato Mena ${ }^{3}$, T.P. Potjer ${ }^{1}$, B.A. Bonsing ${ }^{2}$, G. Klöppel ${ }^{4}$, \\ C. Schicker ${ }^{1}$, I. Esposito ${ }^{4}$, V. Fendrich ${ }^{1}$, D.K. Bartsch ${ }^{1}$ \\ ${ }^{1}$ Philipps-Universität, Marburg, ${ }^{2}$ Leiden University \\ Medical Center, Leiden, Germany; ${ }^{3}$ Ramon y Cajal \\ University Hospital, Madrid, Spain; ${ }^{4}$ Technical University \\ Munich, München, Germany
}

Background: Pancreatic ductal adenocarcinoma (PDAC) has a poor prognosis and a 5 -year survival rate of only $5 \%$. Hereditary factors play a role in the development of PDAC in $3-5 \%$ of all cases. Surveillance of high-risk groups may facilitate detection of PDAC at an early stage, allowing curative surgical treatment. The aims of this study were: (1) to assess whether surveillance aids detection of early stage PDAC or relevant precursor lesions; (2) to evaluate whether surveillance leads to an improved prognosis.

Materials and Methods: Screening outcomes were collected from three European expert centres that conduct prospective screening programs in high-risk groups. These groups included families with clustering of PDAC, referred to as familial pancreatic cancer (FPC), or families with a gene defect that predisposes to PDAC, e.g. CDKN2A, BRCA2 and PALB2. The surveillance program consisted of annual MRI and/or EUS.

Results: A total of 418 individuals participated in the surveillance programs, including 179 CDKN2A mutation carriers, 214 individuals at risk for FPC and 22 BRCA 1/2 or PALB2 mutation carriers. PDAC was detected in 13 (7.3\%) of the 179 CDKN2A-mutation carriers. The resection rate was $75 \%$ and the 5 -year survival rate was $24 \%$. Two CDKN2Amutation carriers (1\%) underwent surgical resection for (low-risk) precursor lesions. Two individuals $(0.9 \%)$ in the FPC cohort had a pancreatic tumour, including one advanced PDAC and one early neuroendocrine tumour. Thirteen individuals with FPC $(6.0 \%)$ underwent surgical resection for a suspected precursor lesion but only four $(1.9 \%$ of the total group) had high-risk lesions, i.e., high-grade IPMN or PanIN3 lesions. Five individuals (2.3\%) were over-treated by surgery for either serous cyst adenoma or focal fibrosis. One BRCA2mutation carrier was found to have a PDAC, another BRCA2mutation carrier and a PALB2-mutation carrier underwent surgery and were found to have moderate risk precursor lesions. No serious complications or deaths occurred as consequence of the program.

Conclusion: Surveillance of CDNK2A carriers is successful, detecting most PDACs at an early resectable stage and thus improving the prognosis. The effectiveness of surveillance in FPC families is less evident. The main effect appears to be prevention of cancer by removal of precursor lesions.

\section{3 \\ Retracted}

54

Surgical Challenges in the Follow-Up after Intestinal and Multivisceral Transplantation

U.A. Gerlach'1, G. Vrakas ${ }^{1}$, P. Friend ${ }^{2}$, A. Pascher ${ }^{1}$, J. Pratschke ${ }^{1}$, A. Vaidya ${ }^{2}$

${ }^{1}$ Charité Universitätsmedizin Berlin, Berlin, Germany;

${ }^{2}$ Oxford Transplant Centre, Oxford, London, UK

Background: Intestinal and multivisceral transplant recipients have often undergone mulitple operations prior to and early after transplantation. In addition, they receive high amounts of immunosupression. Thus abdominal surgery at a later stage posttransplant is very challenging and may increase the morbidity and mortality of these fragile patients.

Materials and Methods: We retrospectively studied 69 patients ( 27 female, 42 male, $37 \pm 9$ years) with isolated intestinal (ITX, $n=48$ ), modified (mMVTX, $n=7$ ) or typical multivisceral transplantation (MVTX, $n=15$ ) in 2 large european transplant centres. 4 grafts included a kidney and 17 included the abdominal wall. Maintenance immunosuppression consisted of either Tacrolimus Monotherapy in 31 patients or a doubel combination of Tacrolimus/MMF, Tacrolimus/Sirolimus, Tacrolimus/Everolimus. We recorded all intraabdominal interventions, which were performed after the first year posttransplant, and subdivided them into 2 groups depending on whether or not they were related to the intestinal graft.

Results: The median follow-up time posttransplant was 8 years [1;15]. 20 intraabdominal operations were reported in 15 patients after a median of 5 years $[1 ; 13]$ posttransplant.

$80 \%$ were emergency operations: Graft-related surgery was required due to graft ischeamia $(n=2)$, adhesive ileus $(n=$ 3 ) and encapsulating ileus $(n=2)$. Surgery for these patients included partial graft resection $(n=5)$, graft explantation $(n=$ 4 ) and intestinal retransplantation $(n=1)$.

Non graft-related operations did not affect the graft or graft function and were: native colon resection due to Volvulus $(\mathrm{n}=1)$, native nephrectomy due to outflow obstruction ( $\mathrm{n}=$ $1)$, kidney transplantation due to CNI-toxicity $(n=1)$, cholecystectomy due to necrotic cholecystitis $(n=1)$, caesarian $(n=$ $1)$, post-mortem kidney/liver donation $(n=1)$.

There were no deaths related to surgery.

$20 \%$ was elective surgery: Incisional herniotomy at the previous stoma-site $(\mathrm{n}=3)$ and a bilateral inguinal herniotomy without incarceration $(n=1)$. 
Abstracts: 133rd Congress of the German Society of Surgery (DGCH)

A conservative treatment was attempted in 4 patients with: rectovaginal fistula in a stapled rectum stump $(n=1)$, neutropenic colitis $(n=1)$ and upper GI-haemorrhage $(n=2)$. The latter 2 patients died.

The median hospital stay following surgery was 27 days [7;210]. Six patients had to undergo repeated surgery (median number of operations $3[2 ; 13])$.

Conclusion: Abdominal surgery in ITX and (m)MVTXrecipients is challenging but feasible. Especially graft-related surgery was accompanied with a high risk of graft-loss but not with mortality. Non graft-related surgery did not affect graft function or longterm survival.

\section{5 \\ Gene Expression Profiles Depend on Tumor Locations in Colorectal Cancer (Left vs. Right vs. Rectum) - Colorectal Cancer Is More Than One Disease \\ M. Maus ${ }^{1}$, D. Hanna ${ }^{2}$, H. Alakus ${ }^{1}$, P.S. Plum ${ }^{1}$, F. Berlth', H. Fuchs', M. Bludau', J.M. Leers' ${ }^{1}$, W. Schröder ${ }^{1}$, P. Grimminger ${ }^{3}$, A. Hölscher ${ }^{1}$, H.J. Lenz ${ }^{2}$ \\ ${ }^{1}$ Abteilung für Allgemein, Viszeral und Tumorchirurgie, Universitätsklinikum Köln, Köln, Germany; ${ }^{2}$ Norris Comprehensive Cancer Center, University of Southern California, Los Angeles, USA; ${ }^{3}$ Klinik für Allgemein-, Abdominal- und Transplantationschirurgie, Universitätsklinik Mainz, Mainz, Germany}

Background: Recent data suggests that CRC from different locations show distinct genetic profiles. Rightsided tumors have a worse prognosis and may have less benefit from targeted therapies. We investigated the tumor locations and genetic profiles (KRAS and BRAF mutation status and ERCC1, TS, EGFR and VEGFR2 mRNA expression) in 580 CRC tumors.

Materials and Methods: FFPE tumor specimen from 580 patients with advanced CRC adenocarcinoma were microdissected and DNA and RNA were extracted. Specifically designed primers and probes were used to detect 7 different base substitutions in codon 12 and 13 of KRAS, V600E mutations in BRAF and the mRNA expression levels of ERCC1, TS, EGFR and VEGFR2 by RT-PCR. These values were analyzed according to tumor location (left vs. right vs. rectum).

Results: BRAF mutations were significantly more common in the right colon (15\%), followed by rectum (3.8\%) and left colon $(2.5 \%)$. KRAS mutations occurred at similar frequencies throughout the colon. Gene expression of ERCC1 was significantly higher in right-sided than left-sided colon tumors in KRAS wild-type colon cancers. The highest expression levels for all genes were seen in rectum. These differences reached significant levels for ERCC1 (rectum vs. right and rectum vs. left, $\mathrm{p}<0.001$ ), TS (rectum vs. left, $\mathrm{p}<0.036$ ) and VEGFR2 (rectum vs. right and rectum vs. left, $\mathrm{p}<0.001$ ).
Conclusion: Tumor location in CRC is associated with specific mutation and expression profiles. Differences in chemosensitivity may be explained by mutation status and mRNA levels in right vs. left CRC. Rectum cancers showed a distinct genetic profile when compared to colon which indicates different tumor biology and may be related to differences in the microflora.

Important Note: Data of this paper has been published in parts in 'The Pharmacogenomics Journal' with the title: 'Distinct gene expression profiles of proximal and distal colorectal cancer: Implications for cytotoxic and targeted therapy.'

\section{6}

\section{In vivo and in vitro Remodeling of Molecular Subtypes of Colorectal Cancer}

\author{
S. Schölch, A. Betzler, L. Nanduri, C. Reißfelder,
} M. Muders, J. Weitz, M. Koch

Universitätsklinikum Carl Gustav Carus Dresden, Dresden, Germany

Background: Large-scale next generation sequencing of colorectal cancer (CRC) has revealed a genetically heterogeneous disease; multiple oncogenic pathways can be altered in CRC. Although it is conceivable that distinct molecular subtypes of CRC may require differential treatment approaches, therapeutic implications have only been established for alterations in the RTK-RAS pathway. Despite the high incidence of CRC, the great number of possible molecular subtypes limits the feasibility of patient stratification by molecular subtypes in clinical trials. Therefore, reliable preclinical models of well-defined molecular subtypes of CRC are required.

As most compounds with proven activity in classical preclinical models such as xenograft models fail to demonstrate their activity in patients, genetically engineered mouse models (GEMM) have recently been introduced. These GEMMs, in which key mutations of the desired tumors are induced specifically in the tissue of interest (leading to genuine mouse tumors as opposed to transplanted tumors) have shown more realistic therapeutic responses.

We here describe a GEMM of CRC which enables us to produce genetically defined molecular subtypes of CRC. In addition, ex vivo organoid culture and molecular analysis of these mouse CRC samples can be performed.

Materials and Methods: After analysis of the TCGA exome sequencing data of 276 clinical CRC samples, we identified the most frequent combinations of oncogenic driver pathways in CRC. To remodel these molecular subtypes of CRC, we interbred mice with conditional alleles of the following genes: APC, KRAS, TP53, ARID1A, FBXW7, SMAD4, PTEN, BRAF, PIK3CA, TP53, INK4A/ARF, CDH1, MSH2. To induce local tumors in vivo, an about $2 \mathrm{~mm}$ distal segment of the colon was 
Abstracts: 133 rd Congress of the German Society of Surgery (DGCH)

locally infected with adeno-cre. Local tumor growth was monitored via colonoscopy.

In vitro, crypt stem cells of the mouse colon were isolated and cultured as organoids in a 3D matrix; to induce malignant transformation, adeno-cre was added to the initial culture medium. Further analysis of the resulting tumor organoids was performed with whole proteome mass spectrometry, microarray expression profiling and other methods.

Results: We were able to reliably activate the following major oncogenic pathways (and combinations thereof) in vitro and in vivo: WNT signaling, TGF- $\beta$ signaling, PI3K signaling, RAS/RAF signaling and P53 signaling. The resulting tumors exhibited distinct molecular and phenotypic differences in vivo and in vitro; survival and metastatic activity differ greatly. 'Druggable' targets (e.g. PIK3CA H1047R) were addressable by specific inhibitors in vitro and in vivo. The different genetic subtypes resulted in distinct histopathological subtypes in vivo and striking differences in morphology, transcriptome and proteome of tumor organoids in vitro. In addition, we demonstrated the cross-activation of genetically unaltered pathways by the activation of other pathways via expression profiling and confirmed the results by wholeproteome mass spectrometry.

Conclusion: We have established a clinically highly relevant GEMM of distinct molecular subtypes of CRC. In addition, colon epithelium of these mice can be oncogenically transformed in vitro to produce genetically-defined and stable tumor organoids of CRC. These models can be used to investigate the influence of genetic alterations on therapeutic response prior to the initiation of clinical trials and may be able to further personalize the treatment of CRC patients.

57

Surgery Is Superior to Antibiotics in the Treatment of Acute, Uncomplicated Appendicitis - Results of a Meta-Analysis of Controlled Trials (PROSPERO 2015: CRD42015016882)

J.C. Harnoss, K. Grummich, P. Probst, I. Zelienka,

C. Müller-Lantzsch, J.M. Harnoss, A. Ulrich, M.W. Büchler, M.K. Diener

Universitätsklinikum Heidelberg, Heidelberg, Germany

Background: For the last few years there has been an ongoing debate discussing a non-operative treatment for acute appendicitis. Compared to the gold standard by appendectomy (OT) some studies now suggest antibiotic treatment (AT) as an equally effective but less risky alternative. The aim of this review was to investigate available evidence regarding efficacy and safety of the two therapeutical options in adult patients.

Materials and Methods: Randomized and non-randomized controlled clinical trials were included. A systematic literature search investigating efficacy and safety of OT and AT was conducted in the Cochrane Library (CENTRAL), Medline, Embase and retrieved reference lists. Risk ratio (RR) or mean difference was calculated with 95 per cent confidence intervals (c.i.) based on intention-to-treat analysis.

Results: Eight studies with 2551 patients were included. Within the first year $26.5 \%$ (348/1312) of the patients primarily treated with antibiosis finally needed appendectomy, resulting in a significantly differing treatment efficacy of $72.6 \%$ vs. $100 \%$ for the AT and OT group, respectively (OR 0.27 (95 per cent c.i. 0.23 to 0.32 ); $p=0.001$ ). Furthermore we found a significant difference in favor of OT in terms of overall morbidity (OR 1.68 (95 per cent c.i. 1.12 to 2.53); $\mathrm{p}=0.01$ ). In randomized trials the length of hospital stay was significantly longer in the AT group (0.27 days (95 per cent c.i. 0.08 to 0.45 ); $\mathrm{p}<0.005$ ).

Conclusion: Appendectomy for acute appendicitis shortens length of hospital stay at significantly superior efficacy and safety. Despite ongoing debates, current evidence supports appendectomy as the gold standard in the treatment of acute appendicitis.

58

\section{Watson MD - Cognitive Computing Getting Medically Certified by Passing the USMLE}

H.G. Kenngott ${ }^{1}$, A. Apitz' ${ }^{1}$, S. Bader ${ }^{2}$, F. Wagner ${ }^{1}$, A. Termer ${ }^{1}$, A. Rettinger ${ }^{2}$, M. Wagner ${ }^{1}$, B.P. Müller ${ }^{1}$

${ }^{1}$ Universitätsklinikum Heidelberg, Heidelberg, ${ }^{2}$ Karlsruher Institut für Technologie, Karlsruhe, Germany

Background: Watson is an artificially intelligent computer system developed by IBM (IBM Inc., Armonk, New York, USA) capable of answering questions posed in natural language and proved its maturity in beating humans in the television quiz show jeopardy. Since then IBM spreads the technology into every industry. In healthcare, Watson's natural language, hypothesis generation, and evidence-based learning capabilities hypothetically allow it to function as a clinical decision support system based on trained domain specific information for use by medical professionals. In this experiment, in order to prove his capabilities, Watson was trained to pass the United States Medical Licensing Examination (USMLE) as part of the final medical exams.

Materials and Methods: In cooperation with IBM, a clinical decision support application using the Watson framework was used. Watson was trained on a custom medical database (corpus) that encompassed both medical textbooks and domain-specific Wikipedia articles. The used corpus contained 100 eBooks of medical literature not older than 2005, and 15.000 medical Wikipedia articles which were filtered for the categories disease, anatomy, traumatology and treatments. In order to logically connect the given information Watson had to be trained with USMLE test questions. USMLE Question 
online databases were selected to supply Watson with sample question-answer pairs. The Watson system was trained with 800 different question-answer pairs. Watson was then taught to format the test questions, then combined the questions with the multiple-choice answers, then compared the received text snippets and finally computed a score of the answer\&rsquo;s plausibility. This approach used similarity measures to compare Watson's output paragraphs to the given multiple-choice answers or their respective derivatives. The used metrics were based on the cosine distance and on the Kolmogorov distance between topic distributions of the paragraphs obtained by Latent Dirichlet Allocation. The preliminary results of the system were generated evaluating 30 not trained USMLE test questions.

Results: The Watson system answered the 30 test questions with 4-8 answer options with an accuracy of 50\% right answered questions (Sensitivity Weighted Score $=0.55$ ). Excluding the picture and figure containing questions, which currently cannot be processed by Watson, the number of right answered text based questions is $80 \%$. The official passing score for the USMLE step 1 is $62 \%$, with a mean score of $71 \%$.

Conclusion: For the first time these results showed the capability of cognitive computing getting close to passing the USMLE and the computer system being close to 'graduating' medical school. Cognitive computing may lead to a paradigm shift on how we handle medical data and patient care in the future.

\section{9}

\section{TNF $\alpha$ and IFN- $\gamma$ from Radiation-Induced Bone Marrow-Derived Mesenchymal Stem Cells Promotes Cells Apoptosis of Colorectal Cancer Cells}

\author{
H. Feng, S. Yin, T.S. Schiergens, W.E. Thasler \\ Klinikum Großhadern, München, Germany
}

Background: Cellular senescence has been shown to strongly increase upon exposure of mesenchymal stem cells to ionizing radiation, but the aim of this study is to investigate the effects of ionizing radiation on the co-culture system of human bone marrow-derived mesenchymal stem cells (hMSC) and human colorectal cancer cells (CRC).

Materials and Methods: hMSC, SW1116, SW620 and HT29 four cell lines were cultured and used in this study. The induction of cytokines secretion in hMSCs after irradiation was analyzed by Elisa array and flow cytometry. Immunofluorescence staining and western-blot were performed to detect different pathway biomarkers. AutoMac separator was used to separate hMSC and CRC automatically.

Results: The 2D co-culture system, flow cytometry showed elevated proportion of CD133+ cells and Lgr5+ cells than control group, which was reported to be cancer stem cell-like cell. Immunofluorescence revealed up-regulated GFAP and Vimentin protein expression in colorectal cancer cells and
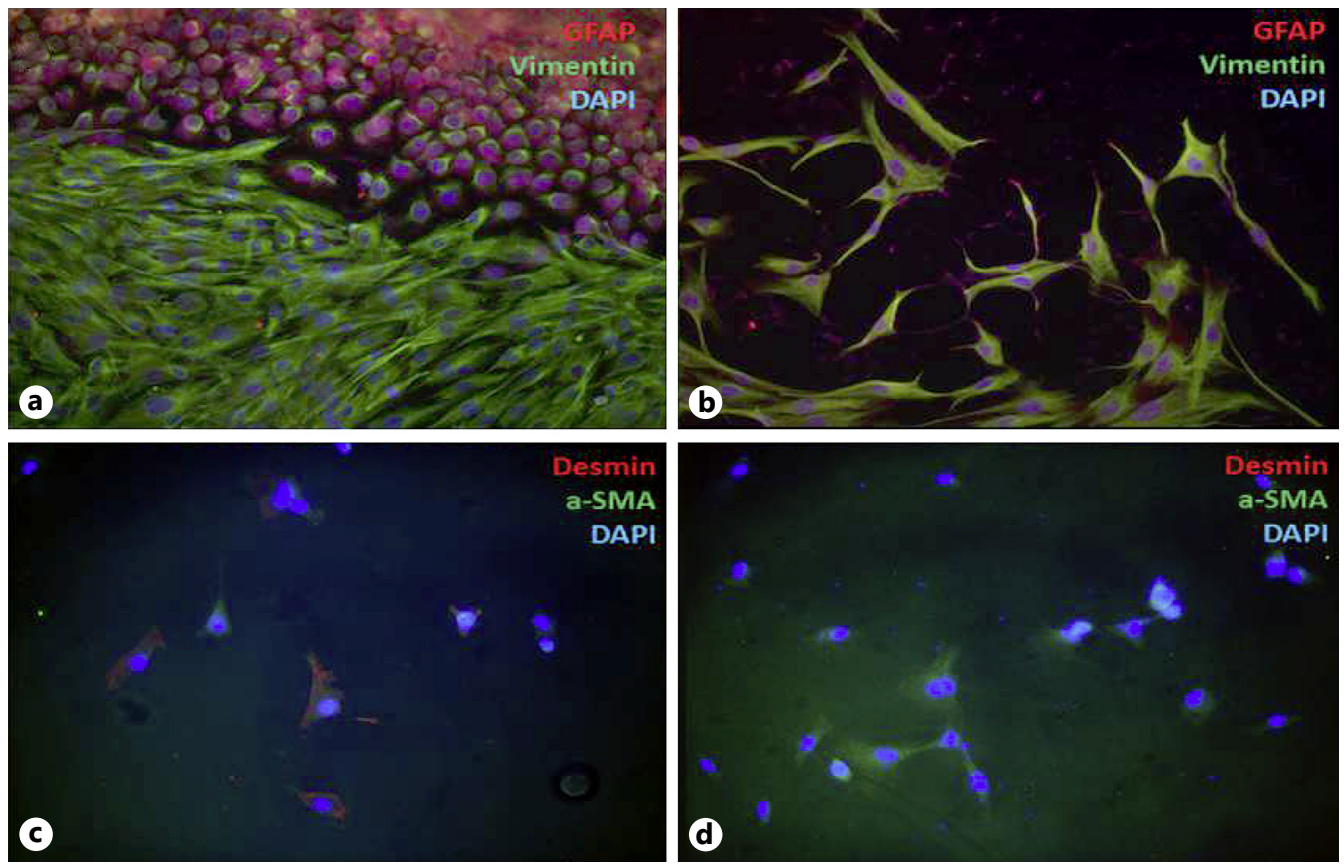

Fig. 1. Immunofluorescence revealed up-regulated GFAP and Vimentin protein expression in colorectal cancer cells and higher $\alpha$-SMA expression level in MSC when co-culture model was established for $48 \mathrm{~h}(\mathbf{a}, \mathbf{b})$, while, the Desmin expression was inhibited (c, d) in MSC, which suggest a fibroblastic differentiation of hMSC (for Abstract 59). 
Abstracts: 133rd Congress of the German Society of Surgery (DGCH)

Fig. 2. Elisa heatmap of supernatant collected from $6 \mathrm{~h}, 12 \mathrm{~h}$ and $24 \mathrm{~h}$ from the co-culture system (for Abstract 59).

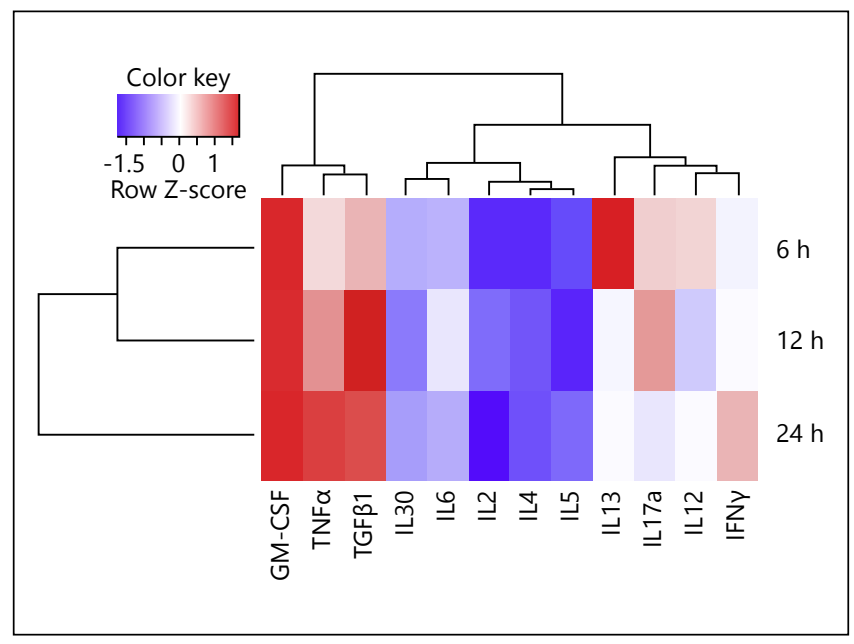

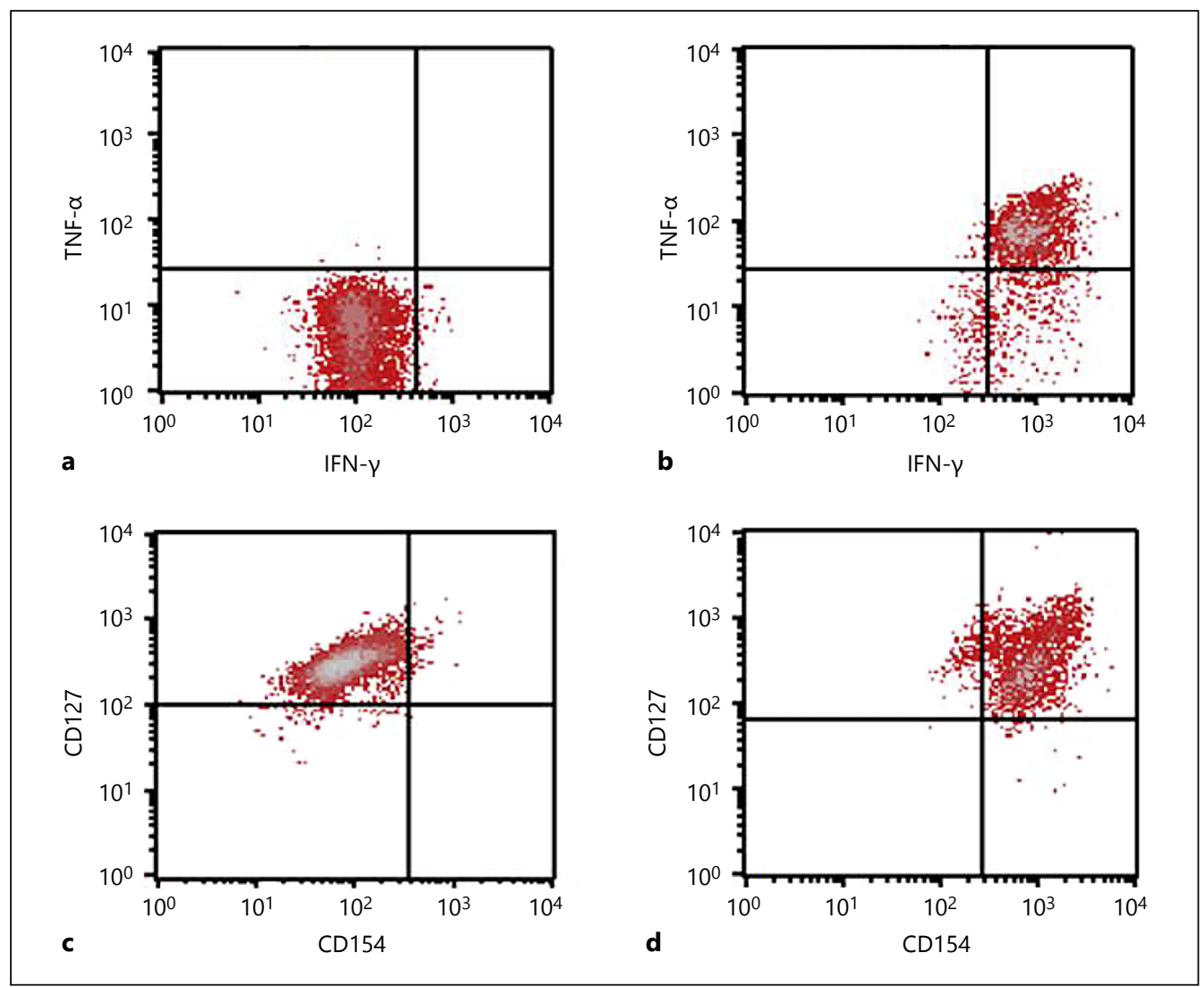

Fig. 3. Flow cytometry of MSC collected from control group (a, c) or co-culture system (b, d) (for Abstract 59).

higher $\alpha$-SMA expression level in MSC when co-culture model was established for $48 \mathrm{~h}$, while, the Desmin expression was inhibited in MSC, which suggest a fibroblastic differentiation of hMSC. The cells of co-cultured group and control group (NC) were then irradiated with a $254 \mathrm{~nm}$ UV-C lamp, prolif- eration assay and viability assay showed inhibited proliferation and less viability of CRC in co-cultured system comparing to the NC group. Western blot reveal up-regulated Caspase3, down-regulated Akt, Erk1/2 in CRC from the co-culture system. The supernatant was collected at $6 \mathrm{~h}, 12 \mathrm{~h}$ and $24 \mathrm{~h}$ to 
Abstracts: 133rd Congress of the German Society of Surgery (DGCH)

be further analysed by Elisa array, which showed increased TNF $\alpha$, IFN $\gamma$, IL4 and depressed IL13. To detect which cell type express TNF $\alpha$ or IFN $\gamma$ flowcytometer was used. We found that after 24 hours of ionizing radiation, MSC rather than CRC expressed higher TNF $\alpha$, IFN $\gamma$, CD154 and IL4, which cause the cytotoxicity against CRC.

Conclusion: Our experiments indicate an increased cell loss rate and the decrease in the tumor cell proliferation activity when irradiated in the co-culture system, as the major mechanisms are MSC secreted TNF $\alpha$, IFN $\gamma$ and IL4.

\section{0}

\section{Impact of Salinomycin on Human Colorectal Cancer Stem Cells in vitro: Inhibition of Spheroid Formation Due to Induction of Apoptosis}

\begin{abstract}
J. Klose, T. Wagner, S. Trefz, A. Preißendörfer Charrier,
T. Schmidt, S. Dieter, H. Glimm, M. Schneider, A. Ulrich

Universität Heidelberg, Heidelberg, Germany
\end{abstract}

Background: The polyether antibiotic Salinomycin (Sal) is regarded as an inhibitor of human cancer stem cells of several solid and non-solid malignancies and therewith as a promising agent for the treatment of cancer in the future. The molecular mechanism of Sal remains unclear. The effectiveness of Sal in colorectal cancer (CRC) stem cells has not been investigated before. Spheroid cultures, obtained from human colorectal cancer specimens, are regarded as cancer stem cells due to similar gene expression profiles to live tumor tissues, self-renewal capacity and tumor initiating potential. This study aimed to investigate the impact of Sal on CRC spheroid cultures and to analyze the molecular mechanism of the drug in CRC.

Materials and Methods: Four different human CRC spheroid cultures were exposed to increasing concentrations of Sal. 5-Fluoruracil (5-FU) and Oxaliplatin (Ox) served as control. The impact of Sal on tumor cell viability (WST-1 assay), stem cell surface marker expression (CD133, CD44 and EpCam) by flow-cytometry, apoptosis induction (SubG1 fraction, AnnexinV expression) and cell cycle was investigated. Regulation on mRNA expression profiles of selected genes involved in tumor cell maintenance, cell death, apoptosis and $\mathrm{Wnt} / \beta$-catenin signaling were analyzed applying quantitative real-time PCR.

Results: Compared to 5-FU and Ox treatment Sal effectively impairs spheroid cell viability in a dose-dependent manner in all tested cell cultures. Spheroid formation was markedly inhibited upon apoptosis induction after exposure to Sal. Alterations in CD133, CD44 and EpCam expression and cell cycle were not observed. Beside several heterogeneous gene expression changes treatment with Sal resulted in a consistent and dose-depending decreased mRNA expression of the intestinal stem cell gene Lgr5.
Conclusion: Sal exerts a toxic effect on human CRC stem cells in vitro. Spheroid formation is inhibited due to apoptosis induction. This has to be regarded as loss of the tumor-initiating potential of the spheroid cultures. This phenotype is accompanied by decreased expression of intestinal stem cell genes including Lgr5. Further in vivo studies have to confirm the results obtained in this study to conduct pre-clinical studies in the future evaluating the potential of Sal in CRC therapy in the future.

\section{1 \\ A Hyperactive CK1 $\delta$ Mutant in Colorectal Cancer Shows Enhanced Oncogenic Potential and Increased Response to Specific Inhibitors}

\author{
J. Bischof ${ }^{1}$ J. Richter ${ }^{1}$, P. Xu1 , D. Vitt ${ }^{2}$, F. Leithäuser ${ }^{1}$, \\ D. Henne-Bruns ${ }^{1}$, M. Kornmann ${ }^{1}$, U. Knippschild ${ }^{1}$ \\ ${ }^{1}$ Universitätsklinikum UIm, UIm, ${ }^{2} 4$ SC Discovery, \\ Planegg-Martinsried, Germany
}

Background: Colorectal cancer (CRC) is the third most common cancer and the fourth leading cause of cancer related death worldwide. Since the 5 year survival rate of metastatic CRC is only about $10 \%$, it is important to receive detailed information on molecular level regarding its carcinogenesis and progression and to search for new effective therapy concepts. Recently, interest in specifically targeting members of the casein kinase 1 (CK1) family has increased. CK1 family members play major regulatory roles in almost all cellular processes including proliferation, apoptosis, and cell differentiation. Considering the importance of CK1 in regulating the functions of tumor suppressors and its contribution in pathways associated with growth and development, it is not surprising that mutations and alterations in the expression and/or activity of CK1 isoforms are often detected in various tumor entities. However, so far the significance of CK1 isoforms in CRC as well as their potential as therapeutic targets in the treatment of CRC has not been addressed in detail.

Materials and Methods: Expression levels of CK1 delta and epsilon $(\delta$ and $\varepsilon$ ) in colon cancer cell lines and tissue specimens of CRC patients were analyzed by using western blotting and immunohistochemistry. Sequencing of CK1 $\delta$ from genomic DNA of CRC patients revealed several point mutations which were introduced into $\mathrm{CK} 1 \delta$-expression vectors and HT2 29 cells. Kinetic parameters of detected CK1 $\delta$ mutants were determined by Michaelis Menten kinetics and effects on cancer cell proliferation were analyzed by using colony formation assay in soft agar. The response to CK1 isoformspecific inhibitors was determined by in vitro kinase assays as well as in cell culture performing MTT viability assays. The oncogenic potential of the detected mutations was also tested in vivo in a subcutaneous mouse xenotransplantation model. Proliferation rates were analyzed using Ki-67 staining. 
Abstracts: 133 rd Congress of the German Society of Surgery (DGCH)

Results: In the present study, we report that human colon tumor cell lines exhibit differences in the expression and activity levels of CK1 isoforms. Inhibition of CK1 $\delta$ by newly developed CK1-specific inhibitors reduce tumor cell proliferation. Furthermore, we show that CK1 $\delta$ and $\varepsilon$ expression is changed in colorectal tumors. Mutations in CK1 $\delta$ were detected in colorectal tumors leading to altered kinetic parameters when being introduced to recombinant CK1 $\delta$ fusion proteins. One mutant (CK1 $\delta$, T67S) displays increased kinase activity in vitro. Overexpression of these mutants in HT29 cells leads to changes in cell proliferation and the ability of cells to grow anchorage independently. Interestingly, the hyperactive mutant shows increased response to treatment with CK1 isoform-specific inhibitors both in vitro and in cell culture. Consistent with cell culture results, introduced CK1 $\delta$ mutations lead to differences in proliferation rate and tumor size in a mouse xenotransplantation model.

Conclusion: In summary, our results provide evidence that colon tumor cell lines and colorectal tumor tissue exhibit differences in expression levels of CK1 $\delta$ and $\varepsilon$. Detected mutations in CK1 $\delta$ affect growth and proliferation of tumor cells. Furthermore, these mutations enhance tumor growth in xenografts, leading to the assumption that CK1 isoforms represent interesting targets in new colorectal cancer therapy concepts, especially because one detected mutants shows increased response to CK1 isoform-specific inhibitors.

\section{2}

Comparative Outcomes of Neoadjuvant Treatment Prior to Total Mesorectal Excision and Total Mesorectal Excision Alone in Selected Stage II/III Low and Mid Rectal Cancer

Y. Kulu, I. Tarantino, A.T. Billeter, M.K. Diener,

T. Schmidt, M.W. Büchler, A. Ulrich

Universitätsklinik Heidelberg, Heidelberg, Germany

Background: Current guidelines advocate that all rectal cancer patients with AJCC stages II and III disease should be subjected to neoadjuvant therapy. However, improvements in surgical technique have resulted in single-digit local recurrence rates with surgery only.

Materials and Methods: Operative, postoperative and oncological outcomes of patients with and without neoadjuvant therapy were compared between January 2002 and December 2013. For this purpose, all patients resected with LAR and TME who had and had not been irradiated were identified from the authors\&rsquo; prospectively maintained database. Patients who were excluded were those with high rectal cancer or AJCC stage IV disease; in the surgery-only group, patients with AJCC stage I disease or with pT4Nx rectal cancer; and in the irradiated patients, patients with ypT4Nx or cT4Nx rectal cancer.
Results: Overall, 454 consecutive patients were included. A total of 342 (75\&thinsp;\%) patients were irradiated and 112 (25\&thinsp;\%) patients were not irradiated. Median follow-up for all patients was 48 months. Among patients with and without irradiation, pathological circumferential resection margin positivity rates (2.9\&thinsp;\% vs. 1.8\&thinsp; \%, $\mathrm{p}=0.5$ ) were not different. At five years, in irradiated compared to surgery-only patients, the incidence of local recurrence was decreased (4.5\&thinsp;\% vs. 3.8\&thinsp;\%, p = 0.5). However, systemic recurrences occurred more frequently (10\&thinsp;\% vs. 17.8\&thinsp; \%, $\mathrm{p}=0.2$ ). Irradiation did not affect overall or disease-free survival (Neo vs. Surg; 84.9\&thinsp;\% vs. 88.2\&thinsp;\%, p = 0.9; 76\&thinsp;\% vs. 79.1\&thinsp;\%, p = 0.8).

Conclusion: The current study adds to the growing evidence that suggests a selective, rather than generalized indication for neoadjuvant treatment in stages II and III rectal cancer.

\section{3}

Effect of Preoperative FOLFOX Chemotherapy on CCL20/CCR6 Expression in Colorectal Liver Metastases

K. Kölsch, C. Rubie, M. Glanemann

Universitätsklinikum des Saarlandes, Homburg, Germany

Background: The combination of chemotherapy and surgery is a promising strategy to improve prognosis in colorectal cancer (CRC) patients. This study evaluated the role of pre-operative chemotherapy with FOLFOX on CCL20/ CCR6 expression in liver metastases in patients with stage IV CRC.

Materials and Methods: Using RealTime-PCR (RT-PCR), enzyme-linked immunosorbent assay (ELISA), Wester Blot (WB) and immunohistochemistry (IHC), we analyzed the expression of CCL20, CCR6 and proliferation marker KI-67 in colorectal liver metastasis (CRLM) specimens from stage IV CRC patients who have received preoperative FOLFOX chemotherapy $(n=53)$ and in patients who have not received FOLFOX chemotherapy prior to liver surgery $(n=29)$. Of the 53 patients who received FOLFOX, time to liver surgery was $\geq 1$ month in 14 patients, $\leq 1$ year in 22 patients and $>1$ year in 17 patients, respectively.

Results: Both CCL20 and CCR6 mRNA and protein expression were significantly increased in patients who have received preoperative FOLFOX chemotherapy $\leq 12$ months before liver surgery $(\mathrm{P}<0.001)$ im comparison to patients who have not undergone FOLFOX treatment. Further, proliferation of CRLM cells as measured by Ki- 67 was increased in patients who underwent FOLFOX treatment.

Conclusion: CCL20 and CCR6 expression were significantly increased in CRLM patients who have undergone preoperative FOLFOX chemotherapy. This up-regulation 
might lead to increased proliferation/migration through an autocrine mechanism which might be used by surviving metastatic cells to escape cell death caused by FOLFOX.

\section{4}

A miRNA Classifier May Predict Metastatic Spread of Colon Cancer and Modulates Target Gene Expression

M. Albertsmeier', S. Heublein ${ }^{2}$, J. Neumann ${ }^{3}$, M.O. Guba', A. Bazhin ${ }^{1}$, T. Kirchner ${ }^{3}$, J. Werner ${ }^{1}$, M.K. Angele ${ }^{1}$

${ }^{1}$ Klinik für Allgemeine, Viszeral-, Transplantations-, Gefäß- und Thoraxchirurgie, München,

${ }^{2}$ Universitätsfrauenklinik Heidelberg, Heidelberg,

${ }^{3}$ Pathologisches Institut, München, Germany

Background: The development of liver metastasis or peritoneal carcinomatosis seems to be mutually elusive in patients diagnosed for colon cancer. We recently identified a stem cell marker profile of the primary tumor to be associated with distant vs. local metastatic spread. In that view, we hypothesize that regarding colon cancer the aforementioned clinical phenomenon may be related to molecular characteristics of the primary tumor itself. To further proof this, we employed miRNA profiling of primary tumor tissue. Those miRNAs being differentially expressed and hence potentially associated with determining the location metastatic spread underwent in vitro validation.

Materials and Methods: A comprehensive miRNA profile ( $\mathrm{n}=754$ miRNAs) was created from tumor tissue stemming from colon cancer patients that had either been diagnosed for liver metastasis (LE; $\mathrm{n}=10$ ) or peritoneal carcinomatosis (PER; $\mathrm{n}=10$ ). Non metastasized cases were used thus to select those miRNAs being triple differentially expressed (LE vs. PER vs. M0) and hence potentially associated with metastatic spread.

Results: Out of the 754 target miRNAs examined we identified 22 miRNAs to be triple differentially expressed. mir215 (16.56-fold), mir31 (5.41-fold) and mir483 (0.04-fold) were most strongly regulated in those primary tumors that presented with peritoneal spread as compared to those that spread to the liver. These three miRNAs also remained to be significantly expressed within validation analysis. Regarding the whole sample mir215 was upregulated in 9 out of 10 and mir31 in $10 / 10$ primary tumors that presented with peritoneal carcinomatosis, while mir483 was only found to be repressed in $6 / 10$ of these cases. Within effector gene analysis, proto-oncogenic c-MET emerged to be targeted by mir31 resulting in repression of c-MET in those cells overexpressing mir31.

Conclusion: In conclusion, this work further extends our previous findings and moreover detected several miRNAs to be differentially expressed in primary tumors of colon cancer patients depending on metastasis localization. We identified mir31 to be induced in those tumors that spread to the peritoneum and to inhibit c-MET. Since c-MET is regarded to be a key regulator of epithelial-mesenchymal-transition it remains to be analyzed whether overexpression of mi31 may functionally alter tumor-biology of colon cancer cells.

\section{5 \\ Isocitrate Dehydrogenase Mutant Diffuse Gliomas Grades II and III Are Radiological Indistinguishable and Underlie Only Molecular Alterations \\ T. Juratli ${ }^{1}$, D. Daubner ${ }^{2}$, A. Zolal ${ }^{2}$, J. Cerhova ${ }^{2}$, K. Engellandt ${ }^{2}, G$. Schackert ${ }^{2}, D . K$ rex $^{2}$ \\ ${ }^{1}$ Universitätsklinikum Carl Gustav Carus an der TU \\ Dresden, Dresden, ${ }^{2}$ Universitätsklinik Dresden, Dresden, Germany}

Background: There is recently increasing evidence that IDH-mutant diffuse gliomas WHO grades II and III - which are still graded based upon light microscopical criteria - share many clinical similarities. Here, we sought to determine whether IDH-mutant diffuse gliomas grades II and III have similar radiological presentations in the magnetic resonance imaging (MRI).

Materials and Methods: Clinical and radiological data of 88 patients; 40 patients with an glioma WHO grade II, 39 patients with glioma WHO grade III and 9 patients with secondary glioblastoma were collected. Sequencing of IDH1 codon 132, IDH2 codon 172 as well as of the TERT-promoter (C146 and C124) was performed. Moreover, the 1p/19q-co deletion status was determined in gliomas harboring oligodendroglial components $(n=32)$. Using immunohistochemistry the MIB- 1 index and the $\mathrm{p} 53$ overexpression status were detected. All molecular data were correlated to the tumor features on MRI before treatment.

Results: IDH mutations were present in 63 patients (79.7\%; in 34 patients with grade II and 29 patients with grade III gliomas, respectively). TERT mutations were detected in $22 \%$ and a co-deleted $1 p / 19 q$ status in $25 \%$ of the entire group. Both groups of glioma grades with an IDH mutation depicted similar radiological features - in regard to tumor size, tumor localization, insular involvement, contrast enhancement, local and/or bilateral infiltration pattern, midline shifting and diffusion restriction - without any significant differences. IDH-mutant gliomas with TERT mutations and/or $1 \mathrm{p} / 19 \mathrm{q}$ co-deletion were predominantly located in the frontal lobe and did not cross the midline nor involve insular structures, making a gross total resection more amenable. A high MIB-1-index and a p53 overexpression were associated with a bigger tumor size and bilateral infiltration of the hemispheres.

Conclusion: Our analysis implicates that IDH-mutant gliomas WHO grades II and III are MR-radiological indistin- 
guishable. We further elucidate that the clinical behavior of diffuse gliomas underlies more likely molecular alterations than the pathological subclasses.

\section{6}

\section{Fluorescent Resonance Energy Transfer Imaging of VEGFR Dimerization}

\section{Yagublu', Z. Ahmadova², T. Förg' ${ }^{2}$, Y. Hajiyeva²,} R. Jesenofsky ${ }^{3}$, M. Hafner ${ }^{2}$, M. Keese ${ }^{4}$

${ }^{1}$ Frankfurt Höchst Hospital, Frankfur, Frankfurt am Main, ${ }^{2}$ Institute for Molecular and Cell Biology, Mannheim,

${ }^{3}$ University Medical Centre Mannheim, Mannheim,

${ }^{4}$ Goethe University Hospital Frankfurt, Frankfurt am Main, Germany

Background: Angiogenesis is a fundamental event in the process of tumor growth and metastatic dissemination. The vascular endothelial growth factor (VEGF) family ligands and their receptors are central in angiogenic signaling. Several strategies, such as inhibition of VEGF ligand or receptor, or even of multiple targets in the downstream signaling pathways have been developed as experimental antitumor strategies. Since receptor homo- and heterodimerization is crucial for VEGF signal transduction, this may represent another interesting target in the search for novel anti-angiogenic agents. Meanwhile, the dominant mechanism of receptor dimerization is not clear: the receptors may be present in an inactive pre-dimerized form, or ligand dependent dimerization may occur. Therefore, we established imaging assays, which allow visualization of receptor dimerization in single intact cells.

Materials and Methods: Microscopic data acquisition was performed on transfected living or fixed CHO-K1 cells. To visualize dimer formation we have employed both a Bimolecular Fluorescence Complementation (BiFC) and Fluorescence Resonance Energy Transfer (FRET) techniques. For the BIFC assay we have developed VEGFR1 - YN173/YC155/ YC173 and VEGFR2-YN173/YC155/YC173 constructs. To determine the effect of VEGF on VEGFR1 homo - and VEGFR1-2 heterodimer formation we performed stimulation and inhibition experiments on co-transfected VEGFR1-CFP and VEGFR1-YFP or alternatively with VEGFR1-CFP and VEGFR2YFP cells applying FRET assay. The transfected cells were either stimulated with different doses of recombinant human VEGF121 or incubated with media containing 10,20 or 100 $\mathrm{ng} / \mathrm{ml}$ VEGF121 in pre-incubation with $10 \mu \mathrm{g} / \mathrm{ml}$ of VEGF inhibitor. Fluorescence microscopy pictures from live cell imaging were used for FRET calculations by sensitized emission (SE-FRET).

Results: The capability to dimerize of the expressed VEGFR-monomers was analysed by a Bimolecular Fluorecence Complemantation (BiFC) assay, using truncated EYFP fragments tagged to the $\mathrm{C}$-terminus of the respective VEGFRs for fluorescence complementation. Fluorescence as an indi- cator of VEGFR dimerization was observed in cells transfected with either VEGFR1 constructs alone or with VEGFR1 and VEGFR2 constructs carrying compatible EYFP fragments, whereas in cells transfected with incomplete EYFP fragments no fluorescence was detected. Combinations resulting in overlapping YFP-fragments (YN172+YC155) demonstrated a bright fluorescence after receptor dimerization. These results further show that the transfected VEGFR constructs are expressed and are able to dimerize either as VEGFR1 homodimers or as VEGFR1/VEGFR2 heterodimers. After receptor dimerization, the fluorophores brought in spatial proximity were expected to undergo FRET. FRET efficiency analyses clearly demonstrated both VEGFR1 homodimerization, and VEGFR1 and VEGFR2 heterodimerization. On the other hand we have shown that VEGFR dimer formation correlates with VEGF stimulation dose and time.

Conclusion: Application of BIFC and FRET imaging techniques, specifically developed and tested along with control sets for the VEGFR1 and VEGFR2 proteins, allowed to monitor the dimerization between different VEGFR subtypes. Both optical assays confirmed the occurrence of VEGFR-1 homodimers and VEGFR1/VEGFR2 heterodimers in intact single cells. The experimental techniques developed in this work will further allow the investigation of similar receptor kinase proteins. Furthermore the techniques can be improved further for monitoring the dynamics of activation processes of the signaling pathways. This may in the future help to develop angiogenesis-targeted drugs for therapeutic purposes in oncologic therapy.

67

\section{3-Dimensional Reconstruction Reveals a Sequence of EMT Events at the Invasive Front of Human Adenocarcinomas}

U.F. Wellner ${ }^{1}$, K. Enderle-Ammour ${ }^{2}$, M. Bader ${ }^{2}$, D. Bausch ${ }^{1}$, T. Keck ${ }^{1}$, P. Bronsert ${ }^{2}$

${ }^{1}$ UKSH Campus Lübeck, Lübeck, ${ }^{2}$ University Medical

Center Freiburg, Freiburg, Germany

Background: Cancer cell invasion is thought to take place at the invasive front. We have recently shown that collective cell migration and partial EMT are the main mode of invasion in various human adenocarcinomas. Tumor budding is a clinical phenomenon with strong prognostic impact defined by small groups of cells disconnected from the main tumor mass at the invasive front of human adenocarcinomas. This study aimed to redefine tumor buds on the basis of branching morphology and cell dissemination.

Materials and Methods: Serial microtome slices from formalin fixed paraffin embedded tissue were used for virtual 3-dimensional reconstruction of the invasive front of human pancreatic, breast, colon and lung adenocarcinoma and stained for PanCK, ZEB1 and E-Cadherin expression. Tumor 
Abstracts: 133rd Congress of the German Society of Surgery (DGCH)

cells were characterized by morphology as polarized, rounded and spindle-like, as well by expression of EMT markers ZEB1 and E-Cadherin. 3D branching morphology was used to define tumor mass branches of increasing order. As an alternative approach, tumor cell cluster size was used to define the amount of cancer cell dissemination.

Results: Quantitative analysis of morphologic changes and EMT marker expression revealed that partial EMT features were not closely associated with the order of tumor branching. On the other hand, decreasing individual cell cluster size turned out to be the strongest determinant of increasing partial EMT features. Using a regression model, a strong inverse correlation of cancer cell cluster size and EMT features was confirmed. Using cutoff numbers from the regression curves, we were able to define a sequence of partial EMT at the invasive front as follows: loss of membranous E-Cadherin, expression of cytoplasmic E-Cadherin, expression of nuclear ZEB1, rounded cell morphology and spindle-like cell morphology.

Conclusion: Cancer cell dissemination into surrounding stroma is accompanied with a sequence of gene expression and morphological changes suggesting partial EMT in rare cancer cells at the invasive front.

\section{8 \\ Is Alpha-2 Macroglobulin an Anti-Cancer Therapeutic Agent Preventing Naked Mole-Rats from Cancer and Permitting Longevity? \\ R. Thieme ${ }^{1}$, I. Gockel', G. Birkenmeier ${ }^{2}$ \\ ${ }^{1}$ Universitätsklinikum Leipzig, Leipzig, ${ }^{2}$ Universität \\ Leipzig, Leipzig, Germany}

Background: The naked mole-rat (NMR) is a long-living and cancer-resistant animal with the size of a mouse, domiciled in the Rift Valley in East Africa. The anti-cancer strategies of the NMR and its longevity are from an eminent interest of ongoing research. The recent finding of a 140 fold higher alpha-2 macroglobulin (A2m) mRNA expression in the liver compared to the short living mouse give evidence to enrol its unusual prevention from age-related diseases. In humans an age-related decrease in A2m blood concentration was shown.

Results: Comparing human and NMR-A2m for its liver and blood plasma abundance we could identify a comparable protein structure of the human and NMR-A2m with an approx. molecular weight of $720 \mathrm{kDa}$. Despite a lower total plasma protein amount $(38.7 \pm 1.79 \mathrm{mg} / \mathrm{mL}$ vs. $61.7 \pm 3.20 \mathrm{mg} / \mathrm{mL})$ in the NMR, we could show a 2 fold higher A2m concentration $(8.3 \pm 0.44 \mathrm{mg} / \mathrm{mL}$ vs. and $4.4 \pm 0.20 \mathrm{mg} / \mathrm{mL})$ in the NMR plasma compared to humans.

Supplementing culture media with 1\% NMR plasma in vitro results in an increase in the adhesion of human fibroblasts and PC-3 cells (prostate cancer). Indeed, adhesion molecules are known for their crucial role in cancer progression, invasion and metastasis, we identified CD29 and CD44 being increased under NMR plasma supplemented in both investigated cells.

Conclusion: In conclusion we hypothesize $\mathrm{A} 2 \mathrm{~m}$ to be a real candidate as a molecule preventing or repressing cancerous processes and promoting longevity by its capability to increase adhesion due to its high abundance in the NMR compared to humans.

Supported by the 'Europäische Sozialfond' - ESF 100098250.
69
Application of Human Mesenchymal Stem Cells as Therapeutic Strategies for Targeting Cholangiocellular Carcinoma - In vitro and in vivo Evidence

\author{
A. Sen Gupta 1 , Y. Zhao ${ }^{1}$, P. Camaj ${ }^{1}$, Y. Wang ${ }^{1}$, \\ D. Augsburger ${ }^{1}$, J. Qin 1 , P. Nelson ${ }^{2}$, C.J. Bruns ${ }^{1}$ \\ ${ }^{1}$ Universitätsklinikum Magdeburg, Magdeburg, ${ }^{2}$ Klinikum \\ der Universität München, München, Germany
}

Background: Cholangiocarcinomas (CCCs) are tumors of the biliary duct system which can be categorized according to their anatomical location in the liver; the majority cases of CCCs are adenocarcinomas and diagnosed in a later stage where they are unsusceptible to any therapy neither curative nor alleviating. The aim of the study is to evaluate whether genetically engineered human mesenchymal stem cells (hMSCs) can improve the therapeutic options for CCCs by using a suicide gene which encodes for herpes simplex thymidine kinase (HSV-tk) under the control of a CCL-5 promotor. Our assumption is that the hMSCs have impact on tumor growth and progression of CCCs by homing into the tumor microenvironment. At the tumor site, the genetically engineered hMSC will express the tk which is activated by Ganciclovir (GCV) and lead cell death through a bystander effect.

Materials and Methods: The interaction effect of hMSCs on human CCC cell lines (CL-6 and HuCCT-1) was detected on proliferation, pro-angiogenic potential, chemosensitivity and apoptosis in vitro. A boyden chamber based system and adhesion assay were applied to evaluated the hMSC homing in vitro. Furthermore we detected the phosphatidylinositol 3-kinase (PI3K)/Akt signalling pathway with a western-blot system in hMSC after tumor cells supernatant treatment. To investigate an in vivo effect, an orthotopic model of CCC was established by tumor cell local-injection into the liver of SCID/ NOD mice. We tested the recruitment of intravenously injected hMSCs and their homing efficacy in this model. For a therapeutic purpose, genetic modified hMSCs transfected with a suicide gene were used.

Results: Our in vitro data showed that hMSCs had a significant effect on the biological function of CCC cell lines. Cell 
Abstracts: 133 rd Congress of the German Society of Surgery (DGCH)

proliferation, pro-angiogenic potential, adhesion and the chemotherapy outcome is vigorous influent by hMSCs. CCC cell supernatant is able to attract MSC transmigration and invasion of hMSC. Moreover it has an up regulating impact on both tAKT and pAKT expression. CCC tumor cells with a high mitotic index (e.g. Cl-6) are more susceptible to external interaction and they showed a stronger ability in manifesting solid tumor in the orthotopic SCID/NOD mice model with lung metastatic potential.

Conclusion: We were able to show that hMSC have a substantial impact on cholangiocarcinoma cells and in the view of the fact that the cholangiocarcinoma has rising incidence there are far more efforts needed to get improved therapeutic options for affected patients. Therefore we will accomplish in vivo experiments on 'homing' of hMSCs as well as potential role of hMSCs on tumor biology will be performed in the SCID/NOD mouse model. In addition, GCV treatment after hMSC administration will be further implemented to analyze whether it could be a possible therapeutic option.

\section{0}

Compliance of Clinical Pathways in Elective Laparoscopic Cholecystectomy: Evaluation of Different Implementation Methods

\author{
S. Müller ${ }^{1}$, C. Flach ${ }^{1}$, R. Hummel ${ }^{2}$, A. Kirschniak ${ }^{1}$, \\ A. Königsrainer ${ }^{1}$, C. Ernst ${ }^{2}$, M. Holderried ${ }^{1}$ \\ ${ }^{1}$ Universitätsklinik Tübingen, Tübingen, ${ }^{2}$ Universität \\ Hohenheim, Stuttgart, Germany
}

Background: Clinical pathways aim to standardize perioperative and postoperative care of surgical procedures and are shown to result in a significant optimzation associated with cost reduction. The aim of this study was to establish the impact of two different implementations forms of clinical pathways on the pathway compliance and resulting costs.

Materials and Methods: Data of patients undergoing elective cholecystectomy for symptomatic cholecystolithiasis were collected over two different periods: using a clinical pathway in form of a clincal pathway integrated into the paper based medical treatment and nursing documentation (group $1, n=123$ ) or a paper based check-list (group $2, n=118$ ). Outcome meassures were compliance of the clinical pathway and total costs per case.

Results: The complete compliance was significantly higher using integrated pathways compared with paper-based check-lists (117 of $123,95 \%$ vs. 54 of $118,46 \%$; p < 0.001). Mean total costs (2206 \&euro; vs. 2458 \&euro; $p=0.027$ ) and length of hospital stay (2.13 days vs. 2.77 days, $p<0.001$ ) were significantly reduced by integrated clinical pathway compared with check-lists. Further the variation of costs per case and variation of length of hospital stay were significantly smaller with integrated clinical pathway ( \pm 440 vs. $\pm 538, p=$ 0.039 and \pm 0.53 d vs. \pm 0.68 d, $p<0.001$, respectively). No difference for postoperative complication was observed (3 vs. 4 events; $\mathrm{p}=0.67$ ).

Conclusion: Integrated clinical pathways display a significant higher compliance compared with checklists resulting in reduced total costs, shorter hospital stay and a smaller variation of cost, making it a useful tool in process controlling and planning.

\section{1}

\section{Surgical Management of Acute Perforated Cholecystitis. A Register Study of Over 5,700 Cases from a Regional Quality Control Database in Germany}

J. Dörner ${ }^{1}$, P. Ambe ${ }^{1}$, S. Macher-Heidrich ${ }^{2}$, H. Zirngibl ${ }^{1}$

${ }^{1}$ Helios Klinikum Wuppertal, Wuppertal, ${ }^{2}$ Ärztekammer

Nordrhein-Westfalen, Düsseldorf, Germany

Background: Acute cholecystitis is a common indication for surgery. Surgical outcomes have been shown to depend amongst other factors on the severity of gallbladder inflammation. Although gallbladder perforation represents the most severe complication of acute cholecystitis, data on the outcomes of patients with gallbladder perforation at cholecystectomy is limited.

Materials and Methods: An analysis of data from a regional quality control database in Nordrhein Westphalia (Germany) was performed. The outcomes of patients undergoing cholecystectomy for acute perforated cholecystitis within a five year period were compared to those of patients without gallbladder perforation.

Results: The study cohort included 45365 patients, of which 5704 had acute perforated cholecystitis (study group) and 39661 cases of acute cholecystitis without gallbladder perforation (control group). The study group was significantly older ( $69.6 \pm 14.2$ years vs. $60.3 \pm 17.5$ years, $p=0.001)$. Surgery lasted significantly longer in the study group $(92.26$ $\min \pm 40.86$ vs. $73.68 \mathrm{~min} \pm 34.10$ ) in comparison with the control group, $\mathrm{p}=0.001$. The rates of morbidity $(10.3 \% \mathrm{vs}$. $3.2 \%, \mathrm{p}=0.001$ ) and mortality ( $4.3 \%$ vs. $1.3 \%, \mathrm{p}=0.001$ ) were significantly higher in the study group compared to the control group. Similarly, the length of stay (13.4 days \pm 11.9 vs. 9.01 days $\pm 8.55, p=0.001$ ) was longer in the study group.

Conclusion: Acute perforated cholecystitis is a severe complication of acute cholecystitis with high rates of morbidity and mortality. Therefore a more aggressive and timely management is needed to improve outcomes. 
Abstracts: 133 rd Congress of the German Society of Surgery (DGCH)

\section{2}

\section{Surgical Management of Empyematous Cholecystitis. A Register Study of Over 12,000 Cases from a Regional Quality Control Database in Germany}

P. Ambe ${ }^{1}$, S. Macher-Heidrich ${ }^{2}$, H. Zirngibl $^{1}$

${ }^{1}$ Helios Klinikum Wuppertal, Lehrstuhl für Chirurgie II, Wuppertal, ${ }^{2}$ Ärztekammer Nordrhein, Düsseldorf, Germany

Background: Acute cholecystitis is a common indication for surgery. Surgical outcomes have been shown to depent amongst other factors on the extent of gallbladder inflammation. Although empyematous cholecystitis represents one of the most severe forms of acute cholecystitis, data on the outcomes of patients undergoing cholecystectomy due to acute empyematous cholecystitis is rare.

Materials and Methods: This is a retrospective analysis of data from a regional quality control database in Germany. Reported cases of acute empyematous cholecystitis within a five-year period between 2010 and 2014 were compared to cases without empyematous cholecystitis with regard to baseline features, clinical parameters and surgical outcomes.

Results: The study was made up of 12,069 patients with empyematous cholecystitis while the control group consisted up 33,296 patients without gallbladder empyema. The study group was significantly older $(67.2 \pm 15.3$ vs. $59.4 \pm 17.6, p=$ 0.001 ) and the percentage of male patients in the study group was significantly higher compared to the control group (53.3\% vs. $45.6 \%, p=0.001$ ). High ASA (scores $>2$ ) were present in significantly more cases in the study group, $\mathrm{p}=$ 0.001 . The study group differed significantly from the control group with regard to fever, elevated white blood count and positive findings on ultrasound sonography, $p=0001$. Surgery lasted significantly longer in the study group (86.1 \pm 38.5 vs. $72.2 \pm 33.6$ ) in comparison with the control group, $\mathrm{p}=0.001$. The rates of conversion ( $15.2 \%$ vs. $5.8 \%)$, bile duct injury ( $0.8 \%$ vs. $0.4 \%)$, re-intervention ( $5.5 \%$ vs. $2.6 \%, p=0.001)$ and mortality ( $2.8 \%$ vs. $1.2 \%$ ) were significantly higher in the study group compared to the control group, $\mathrm{p}=0.001$. Similarly, the length of stay (11.9 \pm 10.5 vs. $8.8 \pm 8.3)$ was significantly longer in the study group, $\mathrm{p}=0.001$.

Conclusion: Empyematous cholecystitis is a severe form of acute cholecystitis with a high rate of mortality. Besides, surgical dissection might be challenging with high rates of complications including bile duct injury.

\section{3 \\ The Clinical Value of Inflammation Markers and BISAP Score in Predicting the Course and the Complications of Acute Pancreatitis}

K. Strupas, A. Sileikis, A. Karpavicius

Vilnius University Hospital, Vilnius, Lithuania

Background: Acute pancreatitis (AP) is a disease with highly variable clinical course. Majority of patients have mild and self-limiting disease, but the mortality rate of patients with severe and complicated forms can reach up to $30 \%$. Early and appropriate treatment will progress in reducing rates of severe AP (SAP) complications and mortality. Delay can be fatal for the AP patient, so early prediction of SAP remains very important. The main problem remains, that neither prognostic scores nor single predictors can\&rsquo;t accurately predict the disease course and complications during the first hours of hospitalization. Therefore, there is a great stimulus for seeking new accurate and easy to use predictors. Perhaps, the least studied group of predictors are adipokines, including adiponectin, leptin, resistin and visfatin. Furthermore, in 2012 the Atlanta classification of AP was revisited and new form moderate severity AP - was indentified. Therefore the criteria of SAP became more stringent and the cut off values of adipokines, so as CRP, IL-6 and BISAP must be recalculated.

Materials and Methods: Objective: to determine prognostic possibilities of adipokines, IL-6, C-reactive protein and BISAP score in predicting the course and the complications of acute pancreatitis. The multicenter study was conducted in Lithuania during the period between April 2012 and March 2015. The diagnosis of AP was established according to the revisited Atlanta classification. Adipokines, IL-6 and CRP levels were measured at admission, as well as the BISAP score was calculated. A contrast-enhanced CT scan was performed on 3rd to 7th day after onset of the symptoms. According to the revisited Atlanta classification, all the patients retrospectively were classified as mild, moderate severity and severe AP cases. The predictive accuracy of each marker was measured by area under the receiver operating curve.

Results: The study enrolled 102 of AP patients (50 males and 52 females, mean age $55.7 \pm 18.1$ years). Mean time after onset of the symptoms was $20.3 \pm 13$.8 hours. The main etiological factors of AP were biliary stones (42.2\%) and alcohol (35.3\%). Necrosis of the pancreas during CECT was detected in $60(58.8 \%)$ patients. According to the revisited Atlanta classification 27 (26.5\%) of all patients were classified as mild, 55 (53.9\%) as moderate severity and $20(19.6 \%)$ as severe AP. 92 $(90.2 \%)$ patients were treated conservatively and 10 (9.8\%) underwent the interventions. Mean length of hospital stay was $20.8 \pm 28.3$ days. Mortality rate was $4.9 \%$.

Resistin (cut-off value $13.7 \mathrm{ng} / \mathrm{ml}$, sens. 63.4\%, spec. 80.0\%, AUC 0.76), IL-6 (cut-off $473.4 \mathrm{pg} / \mathrm{ml}$, sens. $82.9 \%$, spec. $75.0 \%$, AUC 0.78) and BISAP score $\geq 2$ (sens. 64.6\%, spec. $100.0 \%$, AUC 0.91 ) were found to be the early markers of SAP. 
Only BISAP score was an early predictor of pancreatic necrosis (cut-off $\geq 2$, sens. $83.3 \%$, spec. $70.0 \%$, AUC 0.80 ), needs for interventions (cut-off $\geq 3$, sens. $84.8 \%$, spec. $70.0 \%$, AUC 0.85) and mortality (cut-off $\geq 4$, sens. $99.0 \%$, spec. $60.0 \%$, AUC 0.87 ). None of analyzed laboratory markers can predict the pancreatic necrosis, needs for intervention and mortality on admission.

Conclusion: The prognostic possibilities of adipokines in AP are limited. Only admission resistin levels serves as an early predictor for SAP. Admission IL- 6 concentration also serves as an early predictor for SAP. BISAP score is more universal and earlier for AP course and complications prediction than adipokines, IL-6 and CRP.

\section{4}

\section{Rates of Malignancy in Branch Duct IPMN Are Underestimated - A Systematic Review and Meta-Analysis of Recent Publications}

M. Heckler, C.W. Michalski, S. Schaefle, R.R. Luketina, S. Fritz, M.W. Büchler, T. Hackert

Uniklinik Heidelberg, Heidelberg, Germany

Background: The risk of malignancy in branch duct IPMN (BD-IPMN) is controversially debated. While the number of diagnosed and treated cases increases year by year, diagnostic accuracy is relatively low. Despite the consensus guidelines of Sendai (2006) and Fukuoka (2012), individual treatment decisions are often difficult. We thus systematically reviewed studies reporting on outcomes after conservative and/or surgical management of cysts that were diagnosed as BD-IPMN prior to treatment.

Materials and Methods: Studies reporting on more than 40 patients and published between 2006 (following the Sendai consensus) and 2015 were included if at least one Sendai/ Fukuoka criterion was used for decision making. Definition of malignancy was assumed for patients revealing high-grade dysplasia or invasive cancer. Pooled diagnostic odds ratios were calculated using the MedCalc software (bvba, Ostend, Belgium).

Results: 12 studies including 1727 patients (650 primarily resected, 1077 primarily conservative treatment) were included. The median follow up time ranged from 25 to 77 months. In the primary resection group, $23 \%$ of the patients without any feature predicting malignancy according to the Sendai consensus had a malignant lesion in the histopathological workup (compared to $34 \%$ of patients that qualified as Sendai positive). In 607 patients that were considered Sendai negative and were initially followed conservatively, resection was finally performed in 122 patients after a median follow up period ranging from $12.7-41$ month. $17 \%$ of these patients had a malignant lesion in histopathological analysis. The pooled diagnostic odds ratio (DOR) for presence of at least one Sendai criterion was 13.09 (random effects, 95\% CI 4.17-
$41.12, \mathrm{p}<0.001$ ), with a considerable level of heterogeneity $\left(\mathrm{I}^{2}=76.6 \%\right)$. Furthermore, 3 studies that evaluated the revised Sendai criteria (Fukuoka) were included. Pooled analysis of 200 patients who underwent surgery without an indication for resection according to the revised guidelines demonstrated that malignancy was found in $22(11 \%)$ cases. The DOR for indication for resection according to Fukuoka was 8.94 (fixed effects, 95\% CI 4.95-16.49, $\mathrm{p}<0.001, \mathrm{I}^{2}=0 \%$ ).

Conclusion: This retrospective analysis suggests that the current consensus guidelines do not safely predict the risk of (future) malignancy in BD-IPMN. A considerable heterogeneity among the studies demonstrates the need for further studies in the field. Until more accurate predictive diagnostic parameters are available, a more aggressive surgical approach should be discussed for such lesions.

75

\section{Open-Label Pilot Phase I/II Study of Hyperthermic Intraperitoneal Chemotherapy (HIPEC) in Addition to Macroscopic Complete Resection (R0/R1) of Pancreatic Adenocarcinoma (PanHIPEC; Eudra-CT Nummer 2015-002288-41)}

S. Beckert ${ }^{1}$, M. Löffler ${ }^{1}$, I. Königsrainer ${ }^{1}$, C. Meisner ${ }^{2}$, C. Jägle ${ }^{3}$, P. Horvath ${ }^{1}$, F. Struller ${ }^{1}$, A. Königsrainer ${ }^{1}$

${ }^{1}$ Chirurgische Universitätsklinik Tübingen, Tübingen,

${ }^{2}$ Universität Tübingen, Tübingen, ${ }^{3}$ Universitätsklinikum

Tübingen, Tübingen, Germany

Background: In pancreatic adenocarcinoma, long-term survival remains poor with 5-year survival rates less than $20 \%$ even after curative resection. Disease recurrence has been reported in the local and regional area (50\%), on peritoneal surfaces (40-60\%) and as hepatic metastases (50-60\%). Because of the anatomic location of the pancreas, wide resection margins are not possible resulting sometimes in R1-resections. Tumor dissemination and implantation within the resection site during surgery might be another possible explanation for the large number of local and regional failures.

Materials and Methods: Hyperthermic intraperitoneal chemotherapy (HIPEC) combined with radical surgery has been shown to be capable of improving survival in selected patients suffering from peritoneal metastases derived from colorectal cancer. The goal of HIPEC is to erase minimal residual tumor and free floating tumor cells. However, this putative survival benefit hast to be weighed up against an increased perioperative morbidity and mortality.

Results: The aim of this phase I/II trial is to evaluate the impact of HIPEC with $1000 \mathrm{mg} / \mathrm{m}^{2}$ gemcitabine for $60 \mathrm{~min}$ in addition to macroscopic complete resection (R0/R1) of pancreatic adenocarcinoma. 30-day mortality was defined as primary endpoint. In case of a 30-day mortality of less than 
$10 \%$, overall survival and disease free survival are going to be investigated in a separate phase III study.

The power to detect a critical event in more than one patient (=patient death) is $>81.5 \%$, when the incidence of the critical event is $>10 \%$ in our patient population. Therefore, a maximum of 16 patients are enrolled for safety evaluation. The study will be stopped if more than one patient experiences a critical event.

Conclusion: Safety and toxicity according to CTCAE 4.0 are defined as secondary endpoints. Study initiation as investigator-initiated trial (IIT) is scheduled for november 2015. The study is supported by a AKF-Grant of the University of Tübingen.

\section{6 \\ Progressive Postresection Program (pPRP) after Pancreatic Resection for Malignancy and Precancerous Lesions}

S. Richter ${ }^{1}$, V. Uslar ${ }^{1}$, N. Tabriz ${ }^{1}$, D. Weyhe ${ }^{2}$

${ }^{1}$ Pius Hospital, Oldenburg, ${ }^{2}$ Pius-Hospital Oldenburg,

Oldenburg, Germany

Background: More than 16,000 people were diagnosed with pancreatic cancer and almost as many died of this tumor in Germany in 2010. The relative 5-year survival rate was $8 \%$ in this group. The age-standardized incidence and mortality rates are almost constant, but due to an aging population there is an increase in the absolute number of cases.

At the time of initial diagnosis only $15-20 \%$ of patients with pancreatic cancer present with a resectable disease.

Due to poor prognosis of patients with pancreatic cancer, it is important to develop concepts to improve quality of life in their remaining lifetime, as success of traditional outcome parameters such as progression free survival or overall survival is very limited.

Materials and Methods: After pancreatic resection, the intervention group (cohort $A, n=30$ patients) takes part in an intensified physiotherapy program consisting of endurance and muscle force exercises. The control group (cohort $\mathrm{B}, \mathrm{n}=$ 30 patients) takes part in the standard physiotherapy of the pancreatic cancer centre, Pius-Hospital, Oldenburg, Germany. Both groups receive dietary counseling and if necessary substitution of endocrine/exocrine pancreatic insufficiency.

Quality of life will be evaluated using the Short Form-8 Health Survey and the European Organization for Research and Treatment of Cancer QLQ-C30/QLQ-PAN26 questionnaires.

The present prospective randomized-controlled intervention study is designed as a pilot study.

Conclusion: The aim of this study is to investigate whether intensive physiotherapy improves quality of life of patients after pancreatic resection. In case of positive results for the intervention group, a (multicenter) study should be performed with appropriate power.
The completion of the progressive postresection program will be done by a structured follow-up after pancreatic resection. In this study, all patients receive an abdominal computer tomography scan for follow-up 6 and 12 months postoperatively.

Trial registration. The trial is registered at the \&lsquo; German Clinical Trials Register\&rsquo; DRKS00006786. Date of registration was october 1st, 2014.

\section{7 \\ Results of the Interim Analysis of the SAWHI-V.A.C. ${ }^{\circledR}$-Study}

\section{Seidel}

Institut für Forschung in der Operativen Medizin der Privaten Universität Witten/Herdecke gGmbH, Köln, Germany

Background: A decision of the Federal Joint Committee Germany states that negative pressure wound therapy is not accepted as a standard therapy with full reimbursement by health insurance companies in Germany. This decision is based on the reports of the Institute for Quality and Efficiency in Health Care, which demonstrated that an insufficient state of evidence regarding the use of negative pressure wound therapy (NPWT) for acute and chronic wounds exists. The aim of the SAWHI-V.A.C. ${ }^{\circledR}$-study is to compare the clinical, safety and economic results of both treatment arms and to generate solid evidence regarding the effectiveness of NPWT.

Materials and Methods: A randomised controlled clinical trial to evaluate the effectiveness and safety of NPWT for the treatment of subcutaneous abdominal wound healing impairment after surgery compared with standard conventional wound therapy has been initiated. The trial is financed and supported by the manufacturer Kinetic Concepts Incorporated (KCI/Acelity). The first patient was randomised in August 2010. After including 278 patients one planned interim analysis was performed to evaluate if there is a significant positive or negative effect for V.A.C. ${ }^{\circledR}$-therapy and the trial should be stopped.

Results: In October 2014 the number of patients for the planned interim anylysis was reached. At the timepoint of the interim analysis no superiority of one treatment arm was demonstrated for the primary endpoint time number of days to achieve complete, sustained and photo-verified wound closure. For the secondary endpoint wound closure rate the number of confirmed wound closures reached within the maximum treatment time of 42 days was higher in the V.A.C. ${ }^{\circledR}$ treatment group than in the standard conventional wound treatment group.

Conclusion: The recruitment continues. The final analysis of the trial will be performed after reaching the a priori calculated overall sample size of 550 patients to be analysed. Overall study results will be provided to contribute to the final decision 
of the Federal Joint Committee Germany regarding the general admission of negative pressure wound therapy as a standard performance within both medical sectors in Germany.

\section{8 \\ Beyond Survival - Results and Functional Outcome Following Operative Treatment of Necrotizing Fasciitis in Adults \\ D. Hutter ${ }^{1}$, J. Gessmann ${ }^{2}$, M. Königshausen ${ }^{2}$, D. Seybold², T. Schildhauer ${ }^{2}$, M. Coulibaly ${ }^{2}$ \\ ${ }^{1}$ Ruhr-Universität Bochum, Bochum, \\ ${ }^{2}$ Berufsgenossenschaftliche Universitätsklink \\ Bergmannsheil GmbH, Bochum, Germany}

Background: Necrotizing fasciitis (NF) is still a life-threatening infection of superficial muscle fascia and adjacent subcutaneous tissue/skin with high mortality and morbidity. However, data regarding functional outcome is limited.

Materials and Methods: Over a ten-year period, 20032012, 69 skeletally mature patients were retrospectively identified with operative treatment of a NF at a Level I teaching trauma center. Outcomes were results and complications, as well as prospective assessment of long-term functional outcome (Short Musculoskeletal Form Assessment [SMFA] and Short Form 36 [SF-36]).

Results: $35 / 69$ (50.7\%) males and 34 females had a mean age of 64 years (28-97) and a BMI of 29.7 (18.3-86.4). NF classification included 20 Type I, 38 Type II, and 2 Type III microbial pattern - 9/69 (13.0\%) patients had no identifiable microbes. Treatment consisted of i.v.-antibiotic therapy in all, 60 debridement, 58 necrosectomy, 41 fasciectomy, and 19/69 (27.5\%) amputations. Mortality rate was 19/69 (27.5\%). Complications included 20 septic shock, 12 renal failure, 10 ARDS, 7 coagulopathy, and 4 multi-organ-failures. Increasing age independently contributed to non-survival $\left(R^{2}=0.127, p=\right.$ 0.003 ) and age above 60 years had a 7.2-fold higher probability for non-survival (OR 95\% CI 1.511-34.705, $\mathrm{p}=0.013$ ). BMI independently contributed to non-survival $\left(R^{2}=0.102, p=\right.$ $0.008)$. Need for amputation was associated with non-survival $\left(\chi^{2}=0.037\right)$.

22/50 survivors returned valid questionnaires. However, survivors of NF showed impaired functional performance in all scores when compared to normative controls ( $p<0.01)$, except for MCS (table 1).

Conclusion: Necrotizing fasciitis remains challenging with regard to optimal medical treatment and requires interdisciplinary interplay of internal medicine, orthopedic surgeon, microbiologist, anesthesiologist, and intensive care physician. Mortality and morbidity in the adult population is

Table 1. Functional outcome scores in patients with NF (for Abstract 78)

\begin{tabular}{|c|c|c|c|c|c|c|c|}
\hline & Mean STD & Range & Normative STD & $\mathrm{n}^{1}$ & $\mathrm{p}$ value & tvalue & $95 \% \mathrm{CI}$ \\
\hline \multicolumn{8}{|l|}{ SF-36 $(n=22)$} \\
\hline Physical functioning & $33.1 \pm 15.31$ & $15-27$ & $85.8 \pm 20.0$ & 9,408 & $<0.001$ & -16.11 & -45.902 to -59.498 \\
\hline Role-physical & $36.3 \pm 11.02$ & $25-56$ & $82.1 \pm 33.2$ & 9,404 & $<0.001$ & -19.29 & -48.875 to -50.725 \\
\hline Bodily pain & $41.9 \pm 12.45$ & $24-63$ & $75.6 \pm 23.0$ & 9,411 & $<0.001$ & -12.65 & -28.163 to -39.237 \\
\hline General health & $38.6 \pm 10.12$ & $22-58$ & $77.0 \pm 17.7$ & 9,395 & $<0.001$ & -17.73 & -33.901 to -42.899 \\
\hline Vitality & $45.5 \pm 9.26$ & $33-63$ & $65.8 \pm 18.0$ & 9,399 & $<0.001$ & -10.24 & -16.181 to -24.419 \\
\hline Social functioning & $43.3 \pm 14.86$ & $14-57$ & $86.2 \pm 19.8$ & 9,408 & $<0.001$ & -13.51 & -36.301 to -49.499 \\
\hline Role-emotional & $39.0 \pm 15.15$ & $24-55$ & $84.0 \pm 31.7$ & 9,406 & $<0.001$ & -13.86 & -38.257 to -51.743 \\
\hline Mental health & $44.5 \pm 14.05$ & $7-64$ & $77.5 \pm 15.3$ & 9,400 & $<0.001$ & -11.00 & -26.734 to -39.236 \\
\hline PCS & $33.3 \pm 12.08$ & $12-56$ & $50.5 \pm 9.0$ & 9,367 & $<0.001$ & -6.67 & -11.841 to -22.599 \\
\hline MCS & $47.1 \pm 12.35$ & $19-63$ & $51.7 \pm 9.1$ & 9,367 & NS & & \\
\hline $\operatorname{SMFA}(\mathrm{n}=22)$ & & & & $n^{2}$ & & & \\
\hline Daily & $45.8 \pm 33.06$ & $3-95$ & $11.85 \pm 19.20$ & 1,891 & $<0.001$ & 4.807 & 19.270 to 48.63 \\
\hline Emotion & $38.4 \pm 24.48$ & $0-89$ & $20.54 \pm 18.38$ & 1,885 & 0.003 & 3.411 & 6.9979 to 28.741 \\
\hline Arm-hand & $19.5 \pm 24.01$ & $0-69$ & $6.02 \pm 12.26$ & 1,890 & 0.016 & 2.629 & 2.822 to 24.138 \\
\hline Mobility & $53.2 \pm 29.32$ & $0-97$ & $13.61 \pm 18.31$ & 1,888 & $<0.001$ & 6.391 & 26.568 to 52.612 \\
\hline Dysfunction & $41.1 \pm 24.27$ & $2-74$ & $12.70 \pm 15.59$ & 1,871 & $<0.001$ & 5.475 & 17.620 to 39.181 \\
\hline Bother & $38.8 \pm 27.56$ & $0-79$ & $13.77 \pm 18.59$ & 1,734 & $<0.001$ & 4.253 & 12.796 to 37.264 \\
\hline
\end{tabular}

STD = Standard deviation; SF-36 = medical outcomes study Short From-36; PCS = physical component summary score; MCS = mental component summary score; NS = not significant; SMFA = short musculoskeletal function assessment.

${ }^{1}$ Hopman WM: CMAJ 200;163:267-71; ${ }^{2}$ Hunsaker FG: JBJS Am 2002;84-A:208-15.

Functional outcome score in adults surviving necrotizing fasciitis compared to normative controls. 
still high. Age and BMI contribute to non-survival. However, patients surviving NF show persistent functional impairment in the long-run. Functional outcome score in adults surviving necrotizimg fasciitis compared to normaltive controls.

79

\section{Arterial Blood Supply of Liver Segment 4 and Its Possible Surgical Consequences}

\section{T. Alghamdi ${ }^{1}$, C. Viebahn ${ }^{2}$, C. Justinger ${ }^{1}$, T. Lorf ${ }^{3}$}

${ }^{1}$ Städtisches Klinikum Karlsruhe, Karlsruhe,

${ }^{2}$ Georg-August-Universität-Göttingen, Göttingen,

${ }^{3}$ Universitätsmedizin Göttingen, Göttingen, Germany

Background: Segment 4 is unlike the other segments in the segmental liver anatomy and the arterial supply to segment 4 has been an area of debate. The risk of ischemia and necrosis of segment 4 after split-liver resection or transplantation is high. The study was done to identify the arterial blood supply of liver segment 4 and its intrahepatic distribution in an anatomical study.

Materials and Methods: The anatomy of segment 4, the intrahepatic blood distribution, and the origin of the middle hepatic artery (MHA) were studied in 29 livers from fresh adult cadavers. After the liver was explanted, the porta hepatis and the portal triad were dissected to reveal the anatomical variations of the hepatic arteries. Then liver capsule of segment
4 was removed. To identify the arterial blood supply of segment 4, water and ink were injected into the various branches of the hepatic artery and the outflow through segment 4 and discoloration of the liver parenchyma was observed.

Results: In 23 of the 29 livers (79.3\%), the arterial perfusion and distribution in segment 4 was separated by a line drawn from the left side of the inferior vena cava at the top and lateral to the falciform ligament to the medial point of the gallbladder bed (figure 1). The area lateral to this line supplied mainly by the right hepatic artery (RHA) and the area medial to it supplied mainly by the left hepatic artery (LHA). The middle hepatic artery (MHA) arose from the RHA in nine livers and from the LHA in a further nine instances giving a frequency of $31 \%$ for each occurrence.

Conclusion: In addition to the classification system of Couinaud depending on the intrahepatic portal branches, we describe here a new division of liver segment 4 based on arterial blood supply. The arterial blood supply coming from the right and the left hepatic artery with a demarcation line between the areas supplied by RHA and LHA. These anatomical findings might be helpful in the definition of the resection line in the ALLPS procedure or for split-liver transplantation to prevent necrosis of segment 4 .

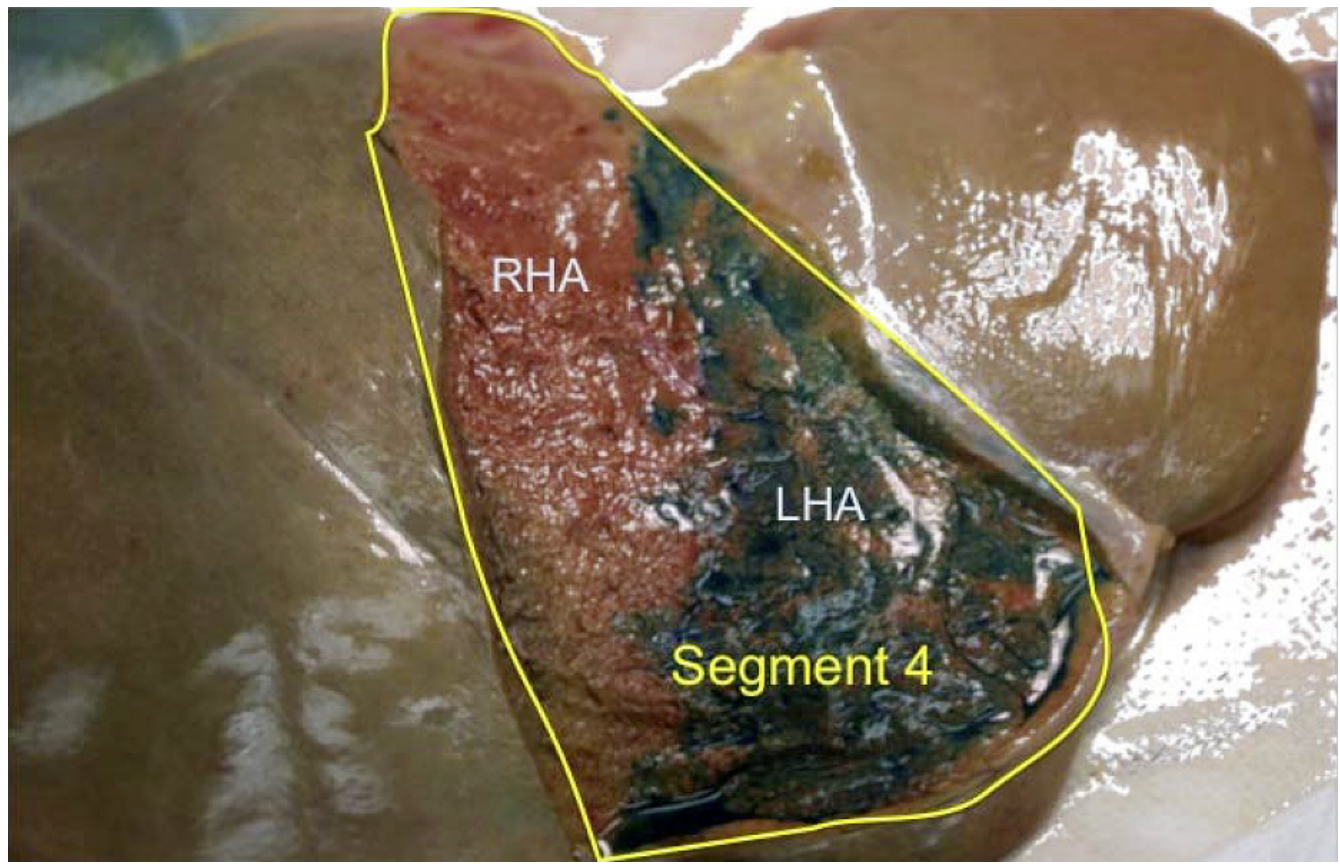

Fig. 1. Demarcation line after the injection of ink in the left heptic artery (LHA), which divide segment 4 (yellow line) according to the arterial blood supply (for Abstract 79). 


\section{0}

Laparoscopic vs. Open Liver Resection: A Single-Center, Retrospective, Matched-Pair Analysis

K. Kouladouros, C. Justinger, D. Gärtner, I. Tournas, M.R. Schön

Städtisches Klinikum Karlsruhe, Karlsruhe, Germany

Background: Since the introduction of laparoscopy in liver surgery in 1992, laparoscopic hepatectomy has gained wide acceptance, especially in specialized, high-volume centers. Until now, a few retrospective, matched-pair studies comparing the postoperative outcomes of laparoscopic and open liver resection have been published. However, in most cases the matched parameters were primarily age, sex, size and localization of the lesions and comorbidities. The aim of our study is to assess the perioperative outcomes of laparoscopic liver resection between two groups matched primarily for the type and extent of hepatic resection.

Materials and Methods: 268 Patients underwent liver resection for benign and malignant disease in our center between 2008 and 2014, of which 80 were laparoscopically and 188 openly resected. A 1:1 matching was applied, based primarily on type and extent of resection and diagnosis. Perioperative outcomes were compared.

Results: After matching, 65 patients were included in each group. There was no statistically significant difference in the baseline characteristics and comorbidities between the two groups. The laparoscopic group showed lower intraoperative ( $p<0.001$ for RBC, $\mathrm{p}=0.019$ for FFP) and postoperative ( $\mathrm{p}<$ 0.001 for RBC, $p=0.021$ for FFP) transfusion rate, shorter hospital stay $(\mathrm{p}<0.001)$ and lower perioperative overall morbidity according to the Dindo-Clavien classification ( $\mathrm{p}<$ 0.001). There were no significant differences in ICU stay, negative margin rate and operative time. 30-day mortality was nil and 90 -day mortality was $1.5 \%$ in both groups.

Conclusion: Laparoscopic liver resection is safe and feasible and offers clear advantages compared to open hepatectomy regarding perioperative transfusion rates, hospital stay and morbidity.

\section{1 \\ Role of Neoadjuvant Chemotherapy in Primary and Recurrent Colorectal Liver Metastasis: A Propensity Score Analysis}

M.J. Strowitzki, T. Schmidt, U. Keppler, S. Mahmoud, J. Klose, M. Schneider, M.W. Büchler, A. Ulrich

Universität Heidelberg, Heidelberg, Germany

Background: About 63,000 patients are diagnosed with colorectal cancer every year in Germany with the liver as the most frequent site of metastasis. In case of liver metastases liver resection remains the only curative treatment. However, especially the role of neoadjuvant chemotherapy in patients with metastases or recurrence of metastases remains controversial. Therefore, the present study aims to investigate the influence of neoadjuvant chemotherapy on the survival of patients with primary and repeated liver resections due to recurrent colorectal liver metastasis (CLM).

Materials and Methods: We retrospectively analyzed 535 patients with CLM who received 693 operations. Patients were separately analyzed for neoadjuvant (NC) and conversion chemotherapy (CC). All patients were divided into four groups: (G1) primary synchronous CLM ( $\mathrm{n}=189$ ), (G2) primary metachronous CLM ( $\mathrm{n}=233)$, (G3) second or repeated resection of CLM ( $\mathrm{n}=138)$ and (G4) patients receiving CC ( $\mathrm{n}=$ 67). Patient data were documented into a prospective database. Finally, propensity score matching (PSM) was performed in all patients without CC $(n=468)$ to reduce selection bias. By this 122 pairs either treated with $\mathrm{NC}$ or without NC could be generated.

Results: Of all 535 patients $36 \%$ (195/535) received NC. In group G1, 36.0\% (68/189), in group G2 27.5\% (64/233), in group G3 36.2\% (50/138) and in group G4 100.0\% (67/67) of the patients received NC. As expected patients receiving NC had significant more non-surgical complications after the operation compared to patients without NC ( $p=0.034)$. Furthermore, patients with NC had a higher Fong-Score and higher number of liver metastases before operation $(\mathrm{p}=0.000$ and $p=0.001)$. Patients with more than one metastasis were more frequent in group $4(\mathrm{p}<0.001)$.

Patients with the first resection of CLM (G1 and G2) did not profit by NC. In group G1, the OS of patients receiving NC was 50.7 months [41.5-60.0 95\% CI] compared to patients without NC with 61.0 months [52.5-69.5 96\% CI]) ( $p=0.242)$. A decrease in OS was also observed in patients with second or repeated resections of CLM (G3) treated with NC with 38.7 months [32.2-45.1 95\% CI] vs. 50.6 months [42.0-59.2 96\% CI] $(p=0.317)$. While patients with a MSKCC score $>3$ showed a significantly reduced OS ( $p=0.004)$, also in these patients, NC did not affect OS.

Expectedly, patients with CC (38.4 months [32.3-44.695\% $\mathrm{CI}]$ ) showed a 22 months shorter OS compared to patients of the other groups (60.2 months [55.2-65.3 95\% CI]) ( $\mathrm{p}=$ 0.148). However, patients receiving CC were not operable before chemotherapy and therefore the survival is long in comparison to palliative treatment.

Before PSM overall survival of all patients without CC was not influenced by NC (NC: 61.2 months [52.0-70.5 95\% CI] vs. no NC: 62.0 months [55.6-68.3 95\% CI]). Interestingly, even after PSM no statistical significant difference in OS between patients with NC and patients without NC could be observed $(\mathrm{p}=0.180)$.

Conclusion: Surgical resection of CLM remains the only curative option. Potentially toxic and harmful neoadjuvant chemotherapy does not affect OS in patients with primary or recurrent CLM while conversion chemotherapy potentially allows a curative resection in previously unresectable patients. 


\section{2 \\ OMANT Pilot Trial - Randomised Controlled Pilot Trial on Feasibility, Safety and Effectiveness of Osteopathic Manipulative Treatment Following Major Abdominal Surgery \\ P. Probst ${ }^{1}$, E. Büchler ${ }^{2}$, C. Doerr-Harim ${ }^{1}$, P. Knebel ${ }^{1}$, A. Ulrich ${ }^{1}$, M.K. Diener ${ }^{1}$ \\ ${ }^{1}$ University of Heidelberg, Heidelberg, ${ }^{2}$ Dresden International University, Dresden, Germany}

Background: Osteopathy, aims at detecting and resolving movement restrictions such as postoperative paralysis. The OMANT Pilot study was the first prospective trial to investigate the feasibility, safety and potential benefits of osteopathic manipulative treatment (OMT) after bowel resection.

Materials and Methods: Twenty Patients with elective bowel resection were randomised in two parallel groups.
Patients in the control group received standard postoperative care. Patients in the intervention group had additional OMT on postoperative days 1 to 5 .

Results: OMANT Pilot was conducted between February and April 2015. Only 2 of 38 patients (5.3\%) were unwilling to participate in the trial. OMT was conducted successfully in 49 of 50 attempts (98\%). OMT patients showed lower postoperative morbidity than control patients (comprehensive complication index 30.8 vs. 37.1). Pain during the postoperative course was significantly lower in the OMT group ( 2 vs. 0 points decrease on numerical rating scale).

Conclusion: Evaluation of OMT in a prospective clinical trial is feasible, and OMT is safe in postoperative patients. Since OMT is a mild, pain-relieving, and well tolerated treatment in surgical patients, it might be beneficial after gastrointestinal surgery, and its effectiveness should be evaluated in an affirmative RCT based on this pilot trial. 